\title{
APPIFICATION, AI, AND HEALTHCARE'S NEW IRON TRIANGLE ${ }^{*}$
}

\author{
NICOLAS P. TERRY**
}

TABLE OF CONTENTS

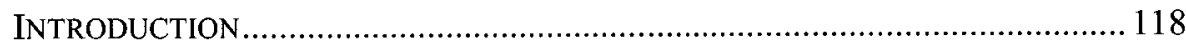

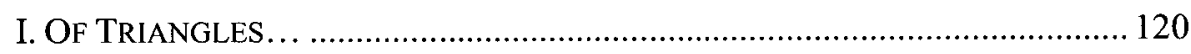

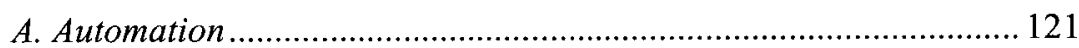

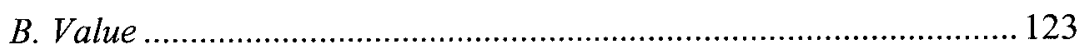

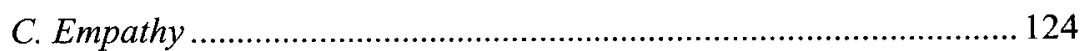

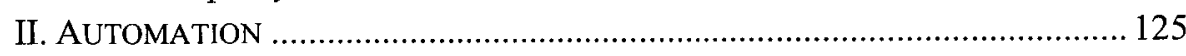

A Technological Tipping Point?...................................................... 126

B. Identifying the Tipping Point: Healthcare's Machine Age ............. 129

1. Personal Health Technologies (Apps and Wearables) ............ 129

2. The Internet of Things....................................................... 131

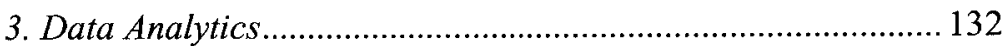

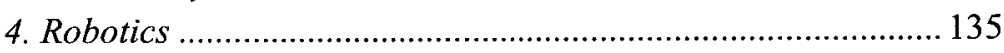

5. Artificial Intelligence and Machine Learning......................... 137

C. Technology Typology and Properties ......................................... 140

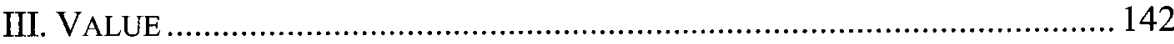

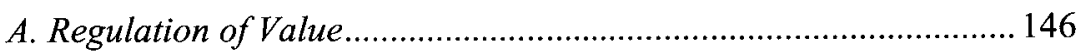

B. Value's Regulatory Failures ..................................................... 149

1. The Professional Distribution Stack .................................... 149

2. Under Regulated Data Protection ...................................... 153

3. Cost-Effectiveness Determination...................................... 157

\footnotetext{
"C 2018 Nicolas Terry. All rights reserved.

**Hall Render Professor of Law, Executive Director, Hall Center for Law and Health, Indiana University Robert H. McKinney School of Law. Email: npterry@iupui.edu. This article is based on the Stuart Rome Lecture I delivered at the University of Maryland Francis King Carey School of Law on April 6, 2017. I am grateful to Diane Hoffmann and Frank Pasquale for this great honor and extend my appreciation to Leslie Francis, Efthimios Parasidis, and Nathan Cortez for their helpful comments.
} 
IV. EMPATHY

A. Will Automation Make Us Rethink Our Conception of Carers and

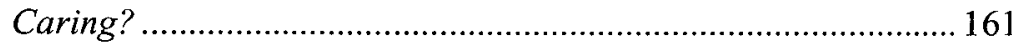

B. Can Automation Handle Provider and Patient Heterogeneity? .... 164

C. Empathy and Equity: Will Machine Age Medicine Benefit All? ..... 165

V. HealthCARE Automation AND APPLyING THE NEW TRIANGle ............ 167

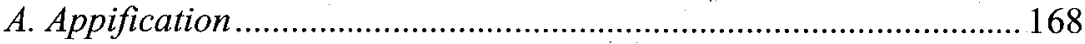

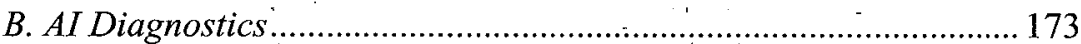

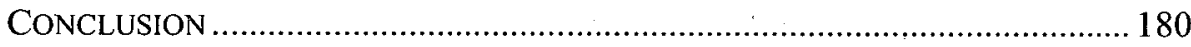

\section{INTRODUCTION}

The relationship between exponentially accelerating technologies and healthcare is simultaneously exciting, challenging, empowering, and (for some) frightening. It has provoked numerous themes in the health law and life sciences law and policy literature. Some scholars have concentrated on the correct regulatory approach to be applied to new health care technologies.' Others have examined the balance between innovation and regulation, exploring how intellectual property and other incentives influence supply side $^{2}$ or demand side behaviors ${ }^{3}$ Still others have surveyed the negative social and economic externalities of emerging health information technologies. ${ }^{4}$

Policymakers tend to reduce healthcare-technology issues to binaries (for example, data liquidity vs. life sciences research or data protection vs. effective clinical care). Yet, the relationships between the various healthcare stakeholders and how emerging technologies impact those relationships or the stakeholders individually are complex and multi-faceted. This complexity will only increase

1. See generally Nathan Cortez et al., FDA Regulation of Mobile Health Technologies, 370 NEW ENG. J. MED. 372 (2014) (discussing the best use of FDA regulatory authority over mobile health technologies); Nathan Cortez, Regulating Disruptive Innovation, 29 BERKELEY TECH. L.J. 175 (2014) (discussing the regulation of new technologies and business practices); Nicolas Terry \& Leslic P. Francis, Ensuring the Privacy and Confidentiality of Electronic Health Records, U. ILL. L. REv. 681(2007) (discussing legal and regulatory steps for electronic inoperable health records to ensure privacy and confidentiality); Nicolas P. Terry, Regulatory Dismiption and Arbitrage in Health-Care Data Protection, YALE J. HEALTH POL'Y L. \& ETHICS 143 (2017) (discussing models of protecting healthcare data).

2.' See, e.g., Rachel E. Sachs, Prizing Insurance: Prescription Drug Insurance as Innovation Incentive, 30 HARV. J.L. \& TECH. 153, 158, 179 (2016) (discussing the potential for prescription drug insurance to remedy a lack of medication treating neglected health conditions).

3. See, e.g., Rebecca S. Eisenberg \& W. Nicholson Price, II, Promoting Healthcare Innovation on the Demand Side, 4 J.L. BIOSCIENCES 3, 4, 6 (2017) (discussing "demand-side innovat[ion]" and its peripheral obstacles and considerations).

4. See, e.g., Frank Pasquale, The Black Box Society: The Secret Algorithms that CONTROL MONEY AND INFORMATION 10 (2015). 
as healthcare assimilates not only patient-facing technologies such as medical apps but also next generation technologies such as robotics and AI.

Healthcare policy choices in this "second machine age" will possess a degree of complexity that will no longer be reducible to policy binaries. Indeed, for students of health law and policy, the level of complexity should recall how we discuss healthcare law and policy generally; by referencing the Iron Triangle of access, quality, and cost containment. Of course, the Iron Triangle lens remains relevant to technologically-mediated healthcare-for example, seeing mobile medical apps as increasing access while containing costs, yet raising questions about quality of care.

However, I contend that the next few decades will see us confronting multifaceted problems that the traditional Iron Triangle does not adequately capture. I propose an additional "New," Triangle to reflect the issues that technologicallymediated healthcare and its stakeholders will be facing. This new triangle's three points are Automation, Value, and Empathy. With this triangle, I hope to provide both an analytical lens and a sorting mechanism for working through the complex issues that will arise. Of course, simply adopting these three new considerations is only the first step; each brings along a subset of issues that, hopefully, will better serve the challenges that lie ahead. Later, I argue that AI healthcare poses some unique challenges. However, some of its other challenges reflect the discussions that have been swirling around technologically-mediated healthcare for the last decade or more; the deprecation of data protection as health data are created or stored outside of traditional healthcare settings, the increasingly difficult task of determining what is a medical "device" in a world ruled by consumer electronics, and the sometimes fraught relationship between healthcare professionals and the machines with which they are forced to interact. ${ }^{5}$

This paper proceeds as follows. First, I reflect on the original Iron Triangle before introducing its young sibling, and discuss their differences, similarities, and intersections. However, just as the points of the original Iron Triangle only begin to tell the healthcare, story so Automation, Value, and Empathy are only the first layer or lens for discussion. Next, I take in order the points of the new triangle, drilling down into some of their properties, suggesting frames for analysis and themes for further study. I conclude by returning to a further comparison of the original and more recent "triangles."

5. See, e.g., Efthimiois Parasidis, Clinical Decision Support, 20 J. HeALth CARE L. \& POL'Y (forthcoming 2018); see also, e.g., Jack M. Balkin, The Three Laws of Robotics in the Age of Big Data, 78 OHIO ST. L.J. (forthcoming 2018); see also, e.g.,Cortez, supra note 1; see also, e.g., Sharona Hoffman \& Andy Podgurski, E-Health Hazards: Provider Liability and Electronic Health Record Systems, 24 BERKELEY TECH. L.J. 1523 (2009); (2014); see also, e.g., Frank A. Pasqualc, Private Certifiers and Deputies In American Health Care, 92 N.C.L. REv. 1661 (2014); see also, e.g., Nicholas P. Terry, Regulatory Disruption and Arbitrage in Healthcare Data Protection, 17 YALE J. HEALTH POL'Y L. \& ETHICS 143 (2016). 


\section{OF TRIANGLES...}

William Kissick's description of his original iron triangle (access, quality, and cost containment) is terse, "All societies confront the equal tensions among access to health services, quality of health care, and cost containment" and that "Tradeoffs are inevitable regardless of the size of the triangle." 6 The triangle's core truth finds expression in his observation that "an expansion of any one angle compromises one or both of the other two." This is immutability laid bare, the recognition that healthcare policymakers always face these trade-offs.

The original triangle is one of the best explanations why healthcare is an

- onerous problem. Consider just two examples that suggest the triangle's legitimacy. First the Affordable Care Act (ACA) did little to live up to its eponym and bend the cost curve. This was because it was skewed towards access (witness the Medicaid expansion and subsidized insurance exchanges). The ACA's most aggressive cost-reducing move was its sanction of accountable care organizations, virtual HMOs, that have yet to prove their triangle-neutering effect. More recently following the election of President Trump, the repeal/replace/repair proposals from the White House and Congress promised a triangle-confounding mixture of better care, cheaper insurance, and overall reduced costs. Alas, those proposals merely signal a return (if we ever fully left it) to what Beatrice Hoffman calls "rationing by price"8-keeping overall healthcare costs relatively stable (in U.S. terms) by treating healthcare as a luxury good that the poor can seldom, if ever access. In both examples, two points of the triangle (Access and Cost) display how most policy shifts improve only one goal and at the expense of another.

The new triangle explores the tension between Automation, Value, and Empathy. As such it seeks to frame the relationship between the upper level properties of healthcare data technologies. As Orly Lobel has discussed, just as Web 3.0 facilitated the growth of platforms for providing (often disruptive) services, so participating firms such as Uber and Airbnb have challenged notions about when and how platforms should be regulated. ${ }^{9}$ There is a distinct whiff of "platform" in the rise of healthcare data technologies. Mobile medical apps "and real healthcare robots (as opposed to the simulacrums of popular fiction) have or will have platform properties. However, all of the technologies discussed here pose or will pose similar questions as to the extent legal principles designed for prior art will take sides between incumbents and disruptive attackers.

\footnotetext{
(1994)

7. Id.

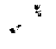

8. BEATRIX HoffMan, Health CARE FOR SOME: RightS AND RATIONING IN THE UNITED STATES SINCE 1930, at 216 (2012) (ebook).

9. Orly Lobel, The Law of the Platform, 101 MiNN. L. REV. 87, 90-91 (2016).
}

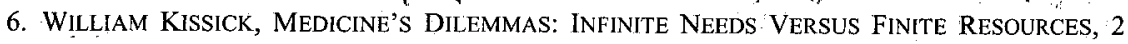


The new triangle does not attempt to directly answer such questions. Rather it suggests a policy framework for approaching those questions. This new construct is driven by a belief that data technologies will increasingly dominate healthcare, fundamentally altering investment priorities, the workforce, and professional interactions with patients. There are, of course, different (and less pretentious) ways to conceptualize some of these issues, and I recognize weaknesses in the new triangle construct. First, it is arguable that an additional triangle is unnecessary - the defect in the original triangle when applied to health data technologies is that it fails to capture the idea of empathy. That defect could be remedied simply by squaring the existing triangle by adding empathy as a fourth element. Even more simply, "Quality" in the original triangle could be read expansively to include empathy.

Both alternates are attractive but fail to deliver much in the way of reframing or the necessary recognition that Automation is about to fundamentally upset our conception of healthcare. Notwithstanding, technical questions about the new triangle remain. There is no doubt that empathy and automation are, or likely will be, in tension just as the three points of the original triangle are. But is Value sufficiently in tension with Automation and Empathy? Certainly, Value (including its safety component) will be in tension with Automation. But what of Value and Empathy? Some aspects of Value, such as safety, seldom will exhibit tension with Empathy. However, other aspects such as the costeffectiveness inquiry will do so; expressing a tension between Value and (patient) values. As I note below ${ }^{10}$ the new triangle is not as immutable as the original, and its points will often nod more towards synergy than tension. It is far more of a thought exercise than the original and assuredly will not have the latter's longevity.

In the larger sections that follow, I describe Automation, Value, and Empathy in detail, outlining what I view to be the more important and/or nuanced properties of each of these points of the triangle. Here, however, I introduce the basic concepts.

\section{A. Automation}

Below I discuss the various types of what I call "healthcare data technologies" and the types of automation they bring to the healthcare space. For the initial purpose of understanding the new triangle however, I also refer to automation in a more normative, even occasionally prescriptive sense. For at least forty years, policymakers and other stakeholders have argued for an increase in the use of information-based (in contrast to clinical) technologies. For example, in 1996 the Health Insurance Portability and Accountability Act ("HIPAA") statute and the regulations that followed pushed the healthcare

10. See infra text accompanying note 366 . 
industry into the world of electronic transactions. ${ }^{11}$ In 2001, the Institute of Medicine ("IOM") argued that information technology ("IT") had a role in improving health care across multiple dimensions; not only safety but also effectiveness, patient-centricity, timeliness, efficiency, and equity. ${ }^{2}$ In 2009 the HITECH Act provided funds to subsidize the purchase of electronic medical records to improve clinical care and care coordination. ${ }^{13}$

Not all of these projects have been successful or popular with healthcare providers or payers. Indeed, value (particularly quality \& safety aspects) and empathy have both been implicated in critiques. In contrast, the enthusiasm of early adopting consumers," policymakers, and technology companies seems palpable, suggesting a sustainable path for innovative healthcare automation. Three examples here: First, the emphasis Apple has put on the wellness, health and health research features of its iPhone and Watch products. Second, the extraordinary acquisition of health records and medically-inflected data by data brokers. Third, the large investment in healthcare projects and services by companies known for their $\mathrm{AI} /$ machine learning, neural networks and algorithms (such as IBM and Google Alphabet).

The original Iron Triangle inevitably led to an apparently never-ending search for a magic bullet to break the unbreakable. That search is what unites ideas such as Accountable Care Organizations, ${ }^{14}$ MACRA's MIPS,,${ }^{15}$ and Section 1332 State Innovation Waivers. ${ }^{16}$ It is easy and appropriate to mock the

11. Health Insurance Portability and Accountability Act of 1996, Pub. L. No. 104-191, §\$261-62 110 Stat. 1,936, 2,021-31 (codified as amended at 42 U.S.C. 1320d (2016)) (creating regulations that require healthcare providers to create secure digital mediums that can be used to facilitate the efficient transfer of sensitive data).

12. Inst. Of Med., Crossing the Quality Chasm: A New Health System for the 21ST Century $164-180$ (Rona Briere ed., 2001).

13. Title XIII (Health Information Technology for Economic and Clinical Health Act, "HITECH") of the American Recovery and Reinvestment Act of 2009 ("AARA"), Pub. L. No. 111-5, 123 Stat. 115, 226-79 (2009) (codified in scattered sections of 42 U.S.C.); see Health IT Legislation, HEALTHIT.Gov, https://www.healthit.gov/policy-researchers-implementers/health-it-legislation (last updated May 12, 2016) (noting the HITECH Act "provides HHS with the authority to establish programs to improve health carc quality, safety, and efficiency through the promotion of health IT, including electronic health records and private and secure electronic health information exchange").

14. See Jenny Gold, Accountable Care Organizations, Explained, KAISER HEALTH NETWORK (Sept. 14, 2015), https://khn.org/news/aco-accountable-care-organization-faq/ ("An ACO is a network of doctors and hospitals that shares financial and medical responsibility for providing coordinated care to patients in hopes of limiting unnecessary spending.").

15. See What's MACRA?, CTRS. FOR MEDICARE \& MEDICAID SERvS., https://www.cms.gov/Medicare/Quality-Initiatives-Patient-Assessment-Instruments/Value-BasedPrograms/MACRA-MIPS-and-APMs/MACRA-MIPS-and-APMs.html (last modified Nov. 16, 2017, 12:32 PM) (explaining the Merit Based Incentive Payments System (MIPS) under the Medicare Access and CHIP Reauthorization Act of 2015 (MACRA)).

16. See generally Heather Howard \& Dan Meuse, State Interest in 1332 Waivers Gaining Steam, HEALTH AFF. BLOG (Nov. 2, 2016), http:/healthaffairs.org/blog/2016/11/02/state-interest-in-1332waivers-gaining-steam/ (reporting state government developments in 1332 waivers to make the Affordable Care Act function better in the state); see also Section 1332: State Innovation Waivers, CTRS. 
comment, "It's an unbelievably complex subject, nobody knew that health care could be so complicated." ${ }^{17}$ However, it is also entirely fair for an observer, whether from Massachusetts, the UK, or the fifth planet orbiting Trappist- $1,{ }^{18}$ to note that the provision of healthcare does not have to be quite so byzantine as the U.S. accidental and dysfunctional model. Couple that with the positive track record of IT solving highly complex problems in other industries and healthcare data technologies look like a realistic solution to those onerous healthcare problems. Inevitably, observations such as these have led to talk of healthcare disruption, a concept based on Schumpeter's model of "creative destruction;" aspirational "industrial mutation...that incessantly revolutionizes the economic structure from within, incessantly destroying the old one, incessantly creating a new one." ${ }^{19}$ Add in the healthcare data technologies examined herein and you have the promise of Clay Christensen's model of "disruptive technologies" that upend incumbents. ${ }^{20}$

\section{B. Value}

This point of the new triangle is the broadest. My starting point is the broad view of "Quality Domains" taken by the Institute of Medicine (IOM). I argue below that some of their inclusions (such as patient centeredness) are more appropriately viewed as part of Empathy. ${ }^{21}$ However, I do agree that the quality domain includes effectiveness, efficiency and (obviously) safety. I also expand the concept somewhat, given the information technologies context to include data protection. Indeed, here quality and safety explicitly include the mandate that data must be safe and of high quality. Specifically, data must be uncorrupted by cyber-breaches, up-to-date, and cross-referenced to evidence bases.

I add an additional layer of abstraction by suggesting that quality, safety, data protection, cost-effectiveness, etc., are part of a still broader concept-

FOR MEDICARE \& MEDICAID SERVS., https:/www.cms.gov/CCIIO/Programs-and-Initiatives/StateInnovation-Waivers/Section_1332_State_Innovation_Waivers-.html (last visited Nov. 27, 2017) ("State Innovation Waivers allow states to implement innovative ways to provide access to quality health care that is at least as comprehensive and affordable as would be provided absent the waiver, provides coverage to a comparable number of residents of the state as would be provided coverage absent a waiver, and does not increase the federal deficit.").

17. Matthew Yglesias, Trump Says "Nobody Knew That Health Care Could Be So Complicated," VoX (Feb. 27, 2017, 11:25 AM), https://www.vox.com/2017/2/27/14750944/trump-health-carecomplicated.

18. See Kenneth Chang, 7 Earth-Size Planets Orbit Dwarf Star, NASA and European Astronomers Say, N.Y. TIMES (Feb. 22, 2017), https://www.nytimes.com/2017/02/22/science/trappist-1-exoplanetsnasa.html (explaining that Trappist-1 is a distant dwarf star with seven planets orbiting).

19. JosePh A. SCHUMPETER, CAPITALiSM, SOCIALISM \& DEMOCRACY 83 (2013) (ebook) (footnote omittcd).

20. Clayton M. Christensen, The Innovator's Dilemma: When New Technologies Cause GREAT FIRMS TO FAIL xv (2013).

21. See infra text accompanying notes 131. 
Value. To express the concept, I adopt the well-known formula, Value $=($ Quality + Outcomes) / Cost. $^{22}$ My normative claim, therefore is that our modern conceptions of quality, safety, and data protection should be further defined and bounded at least by comparative effectiveness research (CER) and preferably by cost effectiveness analysis (CEA).

The Value inquiry involves framing or re-framing regulatory questions in the healthcare data space. Who are the stakeholders and what are their needs and expectations? When do we regulate emerging technologies; at the leading edge, trailing edge, or somewhere in the middle? What are the best models for regulating such technologies, notice and comments, sub-regulatory guidance, or private ordering? To avoid stumbling over every piece of administrative law scholarship from the last two decades I want to emphasize just a couple of issues. First, where do we appear to have under-regulation or overregulation - of healthcare data technologies? In this context, I will be examining the negative effects of what I call the professional distribution stack. Second, I want to examine what I believe to be a critically under-examined characteristic of quality-cost-effectiveness.

\section{Empathy}

The final point of the new triangle is empathy. It was also the most difficult to conceptualize. With empathy, I attempt to capture the aspects of healthcare most valued by pre-patients and patients. The properties captured here include compassion, responsiveness, patient-centeredness, equity, and trust. In addition, empathy values may include other, often unspoken values shared by individual patients and providers.

Overall, these are values that are simultaneously important and very difficult for machines and humans to capture or emulate. As will be discussed below, empathy is also a property that is viewed as one of the last that will be automated..$^{23}$ Empathy also incorporates aspects of autonomy. As such it overlaps with the data protection and consent properties of the Value point of the triangle. However, empathy as used here, goes beyond the somewhat technocratic values of quality, safety, and data protection. Indeed, as noted by ethicists Tom Beauchamp and James Childress, "A rights-based or obligation-based account may neglect appropriate forms of empathy because of its focus on protecting persons from wrongdoing by others. ${ }^{, 24}$ The properties of empathy also offer a

22. See Value $=$ Quality + Outcomes) \%.Cost, MANAGEd CARE (Aug. 2015), https://www.managedcaremag.com/archives/2015/8/value-quality-outcomes-cost (explaining that the value-based care formula is focused on enabling consumers to get the biggest health bang for their healthcare buck and steering the industry away from a high-volume model to a value centric model).

23. See infra Part V.

24. TOM L. Beauchamp \& James F. Childress, Principles of Biomedical Ethics 36 (7th ed. 2013). 
counter to some of the more instrumental values inherent in automation and in some data protection models (in quality and safety).

An automation property such as data determinism typically would be in direct opposition to empathy. Yet, empathy is not always oppositional to automation. Take, for example, a data-mining healthcare bot that automates the calendaring of hospital appointments. A bot which is well provisioned data and possessing sophisticated algorithms should be able to determine where a patient is travelling from and her mode of transportation. It should also be able to learn from the patient's previous arrival times whether she tended to arrive early or late and, from the patient's clinical record, learn about her physical condition or medical constraints (e.g., medication or dialysis times). The bot should then be able to suggest the most convenient appointment time from the perspective of the patient, albeit at the cost of some data protection externalities.

\section{Automation}

As is the case with the other new triangle points, Automation could be labelled differently; maybe healthcare data technologies or even AI. Labels, however, must not obscure the core healthcare Automation proposition. Historically, humans have entered into symbiotic intimate relationships with those with more (or more perceived) knowledge or expertise. We have allowed them to monitor our physical and mental states in the hope that their generalized knowledge can be personalized to improve or sustain our condition. At various points in our history we have made these arrangements with healers, shamans, priests, medicine men, mystics, quacks and sellers of snake oil, doctors and, most recently, healthcare institutions such as hospitals and insurers. Automation is poised to make the most persuasive case yet for such an intimate relationship with our minds and bodies. One must not be naïve here. These technologies will be a forceful presence in our healthcare future. And healthcare necessarily will change as a result. As Kevin Kelly notes, "To demand that artificial intelligence be humanlike is the same flawed logic as demanding that artificial flying be birdlike, with flapping wings. Robots will think different. ${ }^{25}$ One final, general point-we have been promised healthcare technological revolutions before; most recently, precision medicine flowing from Human Genome Project. It was only a few years thereafter that questions began to be raised as to where were the critical mass of practical improvements. ${ }^{26}$ The simple answer is that precision and personalized medicine is still in its infancy. Of course, pharmacogenetics is already delivering operational results suggesting that patients with certain genetic profiles are, for example, better candidates for drug A than drug B.

25. Kevin Kelly, Better Than Human: Why Robots Will-And Must-Take Our Jobs, WIRED (Dec. 24, 2012, 6:30 AM), https://www.wired.com/2012/12/ff-robots-will-take-our-jobs/.

26. See Aaron Rowe, Where's My Personalized Medicine?, WIRED (Mar. 6, 2007, 12:00 PM), https://www.wired.com/2007/03/wheres-my-personalized-medicine/. 
However, it may be decades before we have true personalized treatments. Healthcare data technologies are likely to produce major positive results in a far shorter time span.

Earlier I discussed the normative aspects of Automation. Here I concentrate more on descriptive and classification tasks. I start by reflecting on the argument that we have reached a tipping point into a new machine age. Second, I survey the technologies that seem to be responsible for including healthcare in this revolution. Third, I suggest a typology for these healthcare data technologies.

\section{A Technological Tipping Point?}

Just as the iron triangle has been the dominant meme in healthcare policy, so Moore's $\mathrm{Law}^{27}$ has dominated discussions relating to the development of computer-based technologies. Viewed in its simplified form that "compunting would dramatically increase in power, and decrease in relative cost, at an exponential pace," ${ }^{28}$ Moore's law not only helps explain the past few decades of increases in automation but also is viewed by many futurists as predictive of the new machine age that awaits us. According to Erik Brynjolfsson and Andrew McAfee, "we're at an inflection point-a point where the curve starts to bend a lot-because of computers. We are entering a second machine age." 29

Of course, it is always difficult to objectively analyze a contemporary state, in large part because of the experience of the cutting edge of the arts, science, or whatever. Occasionally, however, there is evidence of a quantum shift. Consider an automobile purchase. Quality and safety are at an all-time high, the handiwork of both markets and regulation. Büt, just how advanced is the modern automobile? Arguably the first modern car was the 1901 Daimler. ${ }^{30}$ It has been viewed as the prototype of modern vehicles. How much changed over the next century? Arguably, relatively little, merely the compounding of incremental improvements, such as electronic ignition, shatterproof glass, disc brakes, radial tires, fuel injection, air bags, anti-lock brakes, etc. All of these improved the

27. See Tom Simonite, Moore's Law is Dead. Now What?, MIT TeCH. REV. (May 13, 2016), https://www.technologyreview.com/s/601441/moores-law-is-dead-now-what/ (explaining that Moore's Law is named after Intel cofounder Gordon Moore who observed "transistors were shrinking so fast that every year twice as many could fit onto a chip.").

28. 50 Years of Moore's Law, INTEL, http://www.intel.com/content/www/us/en/siliconinnovations/moores-law-technology.html (last visited Sept. 12, 2017).

29. ERIK BRyNJOLfSSON \& ANDREW MCAFEe, THE SECONd MACHINE AGE: Work, Progress, AND PROSPERITY IN A TIME OF BRILliANT TECHNOLOGIES 9 (1st ed. 2014) (ebook).

" 30. See Automobile History, HISTORY (2010), http://www.history.com/topics/automobiles ("The 1901 Mercedes, designed by Wilhelm Maybach for Daimler Motoren Gesellschaft, deserves credit for being the first modern motorcar in all essentials."); see also John Clark, Mercedes-Benz History: The . Mercedes $35 \mathrm{HP}$ Super Sports Car, EMERCEDEsBenz (May 14,;,2010), http://www.emercedesbenz.com/autos/mercedesbenz/motorsports/mercedes-benz-history-the-mercedes35-hp/ ("The first modern car was designed by Wilhelm Maybach, chief designer of Daimler-MotorenGesellschaft, in 1900."). 
automobile as we know it, but did not constitute a tipping point. Today, however, we know that the automobile of the next decade is going to be different by an order of magnitude. Tesla, ${ }^{31}$ Waymo, ${ }^{32}$ and other data technology companies ${ }^{33}$ are transitioning automobiles to fundamentally different products, computers with wheels. As noted by Vinod Khosla, "Most commercial flying is now done by auto-pilot, not by the captain." 34 Very soon most driving will be the responsibility of autonomous systems not the driver. The question is; can the same "tipping point" argument can be made for healthcare?

Arguably one can date healthcare from when medicine ceased being just about comforting the dying and we developed the first conceptions of social insurance. ${ }^{35}$ If so, healthcare as we know it is about the same age as the automobile and the airplane. From that time, healthcare technologies (such as drugs and scanning) and outcomes have improved dramatically across multiple dimensions. For example, over the last 50 years the mortality risks associated with anesthesia have declined tenfold. ${ }^{36}$ During the 20th century the U.S. infant mortality rate declined by more than $90 \%{ }^{37}$ and subsequently fell by another $12 \%$ in the first decade of this century. ${ }^{38}$ However, are we convinced that healthcare has out-developed the 1901 Mercedes, that our current healthcare delivery system is radically different from that of 50 years ago?

In the interim, non-healthcare businesses have integrated data technologies throughout their businesses, from internal communications to customer relations and supply chain management. Frequently, they are pushed to do more by disruptive competitors beginning to provide cheaper, more convenient alternatives to their products or services. Data technologies increase coordination

31. TESLA, https://www.tesla.com/ (last visited Nov. 27, 2017); see also Fred Lambert, First Picture of Tesla's New NVIDIA Onboard Supercomputer for Autopilot Installed in a Car, ELECTREK (Jan. 20, 2017, 8:22 AM), https://electrek.co/2017/01/20/first-picture-of-teslas-new-nvidia-onboardsupercomputer-for-autopilot-installed-in-a-car/ (demonstrating how Tesla is using supercomputing to fundamentally change what automobiles are, essentially transitioning them into computers on wheels).

32. WAYMO, https://waymo.com (last visited Sept. 9, 2017).

33. See, e.g., Alex Webb \& Emily Chang, Apple CEO Tim Cook Finally Lifts Lid on Self-Driving Car Project, INDEPENDENT (June 14, 2017, 7:30 http:/www.independent.co.uk/news/business/news/apple-sclf-driving-cars-tim-cook-ceo-launchdriverlessautonomous-system-tech-ai-a7788871.html.

34. Vinod Khosla, Technology Will Replace $80 \%$ of What Doctors Do, FORTUNE (Dec. 4, 2012), http://fortune.com/2012/12/04/technology-will-replace-80-of-what-doctors-do/.

35. See Paul Starr, The Social Transformation of American Medicine 242-43 (1982) (describing the development of the healthcare industry in the early 20th century).

36. See Danicl Bainbridge et al., Perioperative and Anaesthetic-Related Mortality in Developed and Developing Countries: A Systematic Review and Meta-Analysis, 380 LANCET 1075, 1077 (2012) (finding a decrease in anesthetic sole mortality risk from 357 per million in the 1970 s to 34 per million in the $1990 \mathrm{~s}-2000 \mathrm{~s})$.

37. CDC on Infant and Maternal Mortality in the United States: 1900-99, 25 POPULATION \& DEV. REV. 821, 821 (1999).

38. Abby Goodnough, U.S. Infant Mortality Rate Fell Steadily from '05 to '11, N.Y. TimEs (Apr. 17, 2013), http://www.nytimes.com/2013/04/18/health/infant-mortality-rate-in-us-declines.html. 
and improve decision making between and within firms by reducing friction and increasing data liquidity, which furthers data sharing and operational transparency. ${ }^{39}$

It may seem harsh (and is somewhat unfair to HIMSS Level 7 hospitals ${ }^{40}$ ), but sometimes it appears that Moore's Law left healthcare untouched, or at least, that healthcare has been unimpressed by information technologies. The explanations are not hard to find. At root this is has been a function of the reimbursement model. Just as healthcare market failures have perpetuated the original iron triangle, so misaligned incentives have impeded the development of health information technology (HIT). While healthcare heavily invests in high technology, it primarily does so primarily when costs are reimbursable; just count the MRIs and Da Vinci robots in your nearby quaternary hospital. Investments in HIT let alone nimbler -data technologies have been lesssignificant. $^{41}$ The last 30 years have seen healthcare stumble around investment in healthcare data technologies with apparent confusion. Legacy systems were - not replaced, ecommerce models were ignored, HIT not adopted. If you want proof as to the horrors lurking in hospital server closets looks at the number of healthcare cybersecurity breaches and hospital ransomware attacks. ${ }^{42}$ Hackers look for the weak and often find them in healthcare. ${ }^{43}$

39. Nicolas P. Terry, Pit Crews with Computers: Can Health Information Technology Fix Fragmented Care?, 14 Hous. J. HEALTH L. \& POL'Y 129, 141-42 (2014).

40. See HIMMS Analytics EMRAM: Electronic Medical Record Adoption Model, HIMMS ANALYTICS,

http://www.himssanalytics.org/sites/himssanalytics/files/EMRAM\%20Criteria\%20Sheet.pdf (detailing the higher technology implementation requirements for achieving the maximum Level 7 hospital information technology score).

41. See Nathan Cortez, The Mobile Health Revolution, 47 U.C. DAVIS L. REv. 1173, 1180 (2014) (arguing the lack of meaningful FDA oversight impedes development of mobile health technologies); see also Nicolas P. Tcrry, Information Technology's Failure to Disrupt Healthcare, 13 NEV. L.J. 722, $723-$ 24, 727-29 (2013) (discussing business related challenges to adoption of updated HIT).

42. See, e.g., Roland Dela Paz, Off-The-Shelf Ransomware Used to Target the Healthcare Sector, FORCEPOINT (Apr. 5, 2017), https://blogs.forcepoint.com/security-labs/shelf-ransomware-used-targethealthcare-sector (noting that even amateur criminals have the capacity to target healthcare); see also Elizabeth Snell, Healthcare Cybersecurity Attacks Rise 320\% from 2015 to 2016. HEALTH IT SECURITY (Feb. 15, 2017), https://healthitsecurity.com/news/healthcare-cybersecurity-attacks-rise-320-from-2015to-2016 (noting 325 large scale PHI data breaches in 2016).

43. See Melanie Evans, International Cyberattack Affects Some Corners of U.S. Health Care, Including Medical" Devices, WALL" ST. "J. (May 15, 2017, 2:50".PM), https://www.wsj.com/articles/international-cyberattack-affects-some-corners-of-u-s-health-careincluding-medical-devices-1494874222 (reporting an. international cyberattack particularly affecting health-care organizations in the United States); see also Lily Hay Newman, Medical Devices Are the Next Security Nightmare, WIRED (Mar. 2, 2017, 10:30 AM), https://www.wired.com/2017/03/medical-devicesnext-security-nightmare/ (describing distressingly poor security of internet-connected medical devices); see, e.g., Heather Landi, Cyber Attack Update: NH-ISAC Issues Petya Mitigations, Nuance Still Down, Heritage Valley Systems Restored, HeALTHCARE INFORMATICS (July 6, 2017), https://www.healthcareinformatics.com/news-item/cybersecurity/cyber-attack-update-nh-isac-issues-petya-mitigations-nuance- 


\section{B. Identifying the Tipping Point: Healthcare's Machine Age}

There is good reason to believe that this rather negative narrative is poised to change thanks to the maturation of several complementary healthcare data technologies. Together, personal health technologies, the Internet of Things, Data Analytics, Robotics, and Artificial Intelligence (AI) are beginning to make for a compelling story of automation.

\section{Personal Health Technologies (Apps and Wearables)}

The fragmentation, financing, systems, data illiquidity, and locations of U.S. healthcare delivery generate considerable friction. Low-hanging examples include making appointments, acquiring information about costs, procedures, and alternatives, policies, and waiting times (whether "on hold" for an appointment or in a physical waiting room), and monitoring vital signs. ${ }^{44}$ Disruptive technologies, therefore, should be a perfect, ATM-like fit if they provide improved patient convenience and allow patients to perform low-level tasks or transactions independently of professionals or their staff. ${ }^{45}$

Mobile health apps and their accessory wearable devices (such as FitBits or the Apple Watch) promise that independence and have the potential to deliver more convenient and personalized care along with improved convenience and lower cost. Some health apps are "provider-facing," delivering services for medical professionals. Others are developed by traditional healthcare providers or their business partners to improve, for example, access to an EHR. While improving convenience those apps are not disruptive. However, the majority of apps and wearables that collect physiological data and thereafter provide counsel or encouragement operate outside of existing models of health care. ${ }^{46}$ Examples of mobile health apps include allergen-predicting apps such as AllergyCast, ${ }^{47}$

still-down (reporting a cyberattack on a global provider of technology, particularly affecting the company's healthcare business).

44. See generally Terry, supra note 41 at $749-56$ (discussing the potential for mobile technologies to address some common inconveniences in the healthcare industry).

45. See Clayton M. Christensen Et Al., Seeing What's Next: using the Theories of INNOVATION TO PREDICT INDUSTRY CHANGE 181 (2004) (discussing how "disruptive innovations" allowed for "caregivers with less training" to provide "more effective care in convenient, less expensive venues.").

46. Nicolas P. Terry \& Lindsay F. Wiley, Liability for Mobile Health and Wearable Technologies, 25 ANNALS HEALTH L. 62, 62 (2016); see generally Cortez, supra note 41 (discussing the current landscape of mobile health technologies, many operating outside the scope of traditional healthcare providers); see generally Nicolas P. Terry, Mobile Health: Assessing the Barriers, 147 CHEST. J. 1429, 1430-33 (2015) (discussing the current landscape of mobile health technologies, many operating in nontraditional healthcare settings).

47. ZYRTEC, https://www.zyrtec.com/allergy-forecast-tools-apps (last visited Sept. 9, 2017). 
AI-based health "assistants" such as Your.MD, ${ }^{48}$ and chronic disease monitors such as Diabetes $+\mathrm{Me}^{49}$ and the Dexcom G5 Continuous Glucose Monitor. ${ }^{50}$

The first generation of mobile apps and wearables have concentrated on the acquisition of fitness and wellness data and relate to the self-quantification movement. The trending direction for the next generation is self-curation of clinical data and social determinants of health data and sharing those data with smart diagnostic services. ${ }^{51}$ Clinical data storage and data protection models have been built around institutional curation of people's data with carve-outs for other institutions (other clinicians, researchers, public health entities, etc.) interested in that data. However, data-sharing (including interoperability) between electronic medical records systems (EMRs) have been problematic, institutional data custodian security has been exposed as inadequate, and patients are unhappy about the data protection model. If technology continues to outstrip regulation, it is possible that pre-patients and patients will reject the institutionalEMR clinical data hub and move their data to secure enclaves ${ }^{52}$ they control and thereafter decide themselves if, how, and when to share data with institutions whose services they wish to engage. Apple offers the highest level of security and privacy on mobile devices ${ }^{53}$ is reported to be working on one such personal curation model..$^{54}$

The ultimate selling point (and disruptive potential) of mobile apps and wearables is their convenience. Mobile healthcare is nonlinear. Rather than being delivered at centralized, frequently inconvenient locations, mobile technologies are streamed when the patient demands them and are delivered to the patient's

48. YOUR.MD, http://www.your.md (last visited Sept. 9, 2017)

49. DIABETES+ME, https://diabetesplusme.com (last visited Sept. 9, 2017).

50. Introducing the Dexcom G5: Mobile CGM System, DEXCOM, http://www.dexcom.com/g5mobile-cgm (last visited Nov. 11, 2017) (detailing Dexcom G5 capabilities); see also Jordan Kahn, Dexcom CEO Talks 'Game Changer' Diabetes Management Coming to Apple Watch, 9TO5MAC (June 21, 2017, 10:31 AM), https://9to5mac.com/2017/06/21/intervicw-dexcom-ceo-apple-watchcorebluetooth-cgm-diabetes/ (discussing the Dexcom glucose monitor's Apple watch compatibility).

51. See Deborah Lupton, The Quantified Self: A Sociology of SelF-Tracking 89 (2016) (discussing the interactions between HIT and users, and how those interactions affect user behavior).

52. See generally iOS Security: iOS 10, APPLE. 7 (Mar. 2017), https://www.apple.com/business/docs/iOS_Security_Guide.pdf (explaining the iOS secure enclave technology).

53. See Christian Lueg, 8,400 New Android Malware Samples Every Day, GDATA SECURITY BLOG (Apr. 27, 2017), https://www.gdatasoftware.com/blog/2017/04/29712-8-400-new-android-malwaresamples-every-day (noting there were "over 3.2 million new Android malware files in 2016"); see also Lucas Mcarian, Android vs iOS Security: Which is Better?, COMPUTERWORLD (Aug. 7, 2017, 3:09 AM), https://www.computerworld.com/article/3213388/mobile-wireless/android-vs-ios-security-which-isbetter.html (discussing the relative merits of iOS and Android security). Apple's rival, the Android operating system, suffered an increase in new malware samples since 2012.

54. See Christina Farr, Apple Is Quietly Working on Turning Your iPhone into the One-Stop Shop for All Your Medical Info, CNBC (June 14, 2017, 8:23 PM), http://www.cnbc.com/2017/06/14/apple-iphonemedical-record-integration-plans.html (reporting that Apple is developing health information capabilities for the iPhone). 
location. In other words, they represent the "death of queuing," 55 or, at least, the hospital waiting room. Further, sophisticated yet inexpensive monitoring of and coaching for chronic conditions may address some of healthcare's fragmentation problems by better coordinating care when patients are handed off between providers. ${ }^{56}$ Finally, apps (or at least most of them) and wearables are available outside of the professional distribution stack. ${ }^{57}$ For that reason, among others they are also far less expensive than traditional, upstream care.

\section{The Internet of Things}

The Internet of Things (IoT) is "an interconnected environment where all manner of objects have a digital presence and the ability to communicate with other objects and people." 58 Connection to networks imbue "ordinary" or everyday objects with tracking, situational awareness, and sensors that can drive analytics. ${ }^{59}$ Crucially, "people will leave a digital trail wherever they go, even if they are not connected to the internet." ${ }^{\prime 60}$

In the health domain IoT interconnectivity should allow mobile apps and wearables to be more aware of their user's environment, make changes to that environment to improve the user's health and expand the ability to exchange useful data, improve context awareness, and initiate actions within apps based on data that are collected and analyzed from the IoT ${ }^{61}$ For example, smart beds will monitor patient biometric data ${ }^{62}$ and alert family members to a lack of adherence to medication. ${ }^{63}$ Apps and devices that receive inhaler-sourced

55. See generally Nicole Kobie, Will the Internet of Things Mean the Death of Queuing?, GUARDIAN (Mar. 31, 2015, 4:09 AM), http://www.theguardian.com/technology/2015/mar/31/will-the-internet-ofthings-mean-the-death-of-queuing (discussing how connected devices may replace waiting in person for services).

56. See generally Terry, supra note 46 , at 1433 (discussing how some applications allow patients to monitor their health and therefore provides a more consistent record with the patient as opposed to across several providers).

57. See discussion infra Section IV.B.1.

58. FED. TRADE COMM'N, INTERNET OF THINGS: PrIVACY \& SECURITY IN A CONNECTED WORLD 1 (2015), https://www.ftc.gov/system/files/documents/reports/federal-trade-commission-staff-reportnovember-2013-workshop-entitled-internet-things-privacy/150127iotrpt.pdf.

59. Michacl Chui et al., The Internet of Things, MCKINSEY Q. (Mar. 2010),

http://www.mckinsey.com/industries/high-tech/our-insights/the-internet-of-things.

60. Data is Giving Rise to a New Economy, ECONOMIST (May 6, 2017),

$\mathrm{http} / /$ www.economist.com/news/briefing/21721634-how-it-shaping-up-data-giving-rise-new-economy.

61. See generally Nicolas P. Terry, Will the Internet of Things Transform Healthcare?, 19 VAND. J. ENT. \& TECH. L. 327, 328-29 (2016) (discussing potential advantages of HIT integrating with the IOT).

62. See, e.g., Transforming Healthcare and Improving Lives, SLEEP NUMBER, http://bamlabs.com/medical/ (last visited Sept. 7, 2017) (explaining a smart bed can monitor heart and respiration trends and overall sleep quality).

63. See, e.g., Your Personal Home Health Robot, PILLo, http://pillohealth.com (last visited Sept. 7, 2017) (describing how a Pillo can store, dispense, and order refills as well as securely manage vitamins and medication). 
warnings of pollutant levels unfriendly to their, say, asthmatic hosts ${ }^{64}$ potentially could reroute an outdoor activity or automatically increase home or automobile filtration. Similarly, just as GPS systems warn drivers of accidents or construction delays, so a patient restricted to limited activity because of heart problems would be routed away from a destination where, say, the only elevator was out of order. ${ }^{65}$

\section{Data Analytics}

"Data is the new oil"66 or, maybe the "new coal." noted by Paul Sonderegger of Oracle: "Data will be the ultimate externality: we will generate them whatever we do." ${ }^{.68}$ Obviously, data are the fuel for all of the healthcare data technologies discussed herein. However, data collection and processing are themselves key technologiès as well as being intimately related to $\mathrm{AI}$ and its machine learning. As Viktor Mayer-Schönberger and Kenneth Cukier put it, "The key is that these [data analytics] systems perform well because they are fed with lots of data on which to base their predictions. Moreover, the systems are built to improve themselves over time, by keeping a tab on what are the best signals and patterns to look for as more data is fed in." have attempted to model the maturity or trajectory of data analytics. ${ }^{70}$ Initially, analytics tends to be descriptive (what happened?), the next level in is diagnostic (why did it happen?), followed by predictive (what will happen?), and finally prescriptive analytics (how can we make it happen, typically through automation?). Beginning at the predictive but clearly at the prescriptive end of

64. See, e.g., Stephanie Kanowitz, Smart Inhalers Help Monitor Air Pollution, GCN (Nov. 10, 2016), https:/gcn.com/articles/2016/11/10/air-louisville.aspx (describing patient collection and use of inhalers can notify others of location and severity of pollution).

65. Terry, supra note 61 at 349-50; see also John R. Quain, Warning! Traffic Jam Straight Ahead, N.Y." $\quad$ (Oct. TIMES $\quad 30, \cdots \cdots 2$ 2008), http://www.nytimes.com/2008/10/30/automobiles/autospecial2/30traffic.html (describing how GPS models from Tomtom, Garmin, and Navigon are bundled with live traffic feeds).

66. Christopher Heine, Under Armour's CEO Just Hinted at How His Company Can Grow to $\$ 7$ Billion by 2018, ADWEEK (Mar. 14, 2016), http://www.adweek.com/digital/under-armours-ceo-justhinted-how-his-company-can-grow-7-billion-2018-170207/.

67. Alex Hern, Why Data Is the New Coal, GUARdian (Sept. 27, 2016, 6:26 PM), https://www.theguardian.com/technology/2016/sep/27/data-efficiency-deep-learning.

68. Data Is Giving Rise to a New Economy, ECONOMIST (May 6, 2017), http://www.economist.com/news/bricfing/21721634-how-it-shaping-up-data-giving-rise-new-economy.

69. ViKTOR MAYER-SCHÖNBERGER \& KENNETH CUKIER, Big DATA: A REVOLUTION THAT WiLL TRANSFORM HOW WE LIVE, WORK, AND THINK 12 (2013).

70. See, e.g., Timo Elliott, \#GartnerBi: Analytics Moves to the Core, Digital Bus. \& Bus. ANALYTICS BLOG (Feb. 14, 2013), http://timoelliott.com/blog/2013/02/gartnerbi-emea-2013-part-1analytics-moves-to-the-core.html (explaining how analytics is now at the core of Gartner's system); see e.g., Chris Nott, A Maturity Model for Big Data and Analytics, IBM BIG DATA \& ANALYTICS HuB (May 26, 2015), http://www.ibmbigdatahub.com/blog/maturity-model-big-data-and-analytics (explaining how big data and analytics maturity model is a well-suited and durable approach). 
the maturity model there is a clear overlap between data analytics and AI/Machine Learning.

Healthcare agencies are already significant users of data analytics. For example, funded by ACA and required by the Small Business Jobs Act of $2010,{ }^{71}$ CMS runs a pre-payment predictive analytics program called the Fraud Prevention System (FPS) against Medicare fee-for-service claims. ${ }^{72}$ The VA's Recovery Engagement and Coordination for Health - Veterans Enhanced Treatment (REACH VET) program uses data analytics to identify veterans with an elevated risk for suicide. ${ }^{73}$ Various techniques are used to discover fraud including pattern tracking, anomaly detection ${ }^{74}$ and link analysis examines "relationships among claims, people and transactions." " Similarly, the Food and Drug Administration (FDA) has several major projects that leverage big data. ${ }^{76}$ For example, Janus (seeing into the past and the future $)^{77}$ combines historical clinical trial data with post-marketing studies and surveillance-based adverse event data. Another project, the Sentinel Initiative, is a post-marketing product safety system for drugs, vaccines, biologics and devices. ${ }^{78}$ Public health

71. Small Business Jobs Act of 2010, Pub. L. No. 111-240, § 4241(b)(1), 124 Stat. 2504, 2599 (codified at 42 U.S.C. $\S 1320 \mathrm{a}-7 \mathrm{~m}(\mathrm{~b})(1)$ ).

72. Press Release, Ctrs. for Medicare \& Medicaid Servs., CMS Cutting-Edge Technology Identifies \& Prevents \$820 Million in Improper Medicare Payments in First Three Years (July 14, 2015), https://www.cms.gov/Newsroom/MediaReleaseDatabase/Press-releases/2015-Press-releasesitems/2015-07-14.html.

73. Press Release, U.S. Dep't of Veterans Aff,, VA REACH VET Initiative Helps Save Veterans Lives: Program Signals When More Help is Needed for At-Risk Veterans (Apr. 3, 2017), https://www.va.gov/opa/pressrel/pressrelease.cfm? id=2878.

74. $I d$.

75. Russ Schreiber, Fighting Fraud with Predictive Analytics and Link Analysis, HEALTHCARE FIN. (Aug. 8, 2012), http://www.healthcarefinancenews.com/news/fighting-fraud-predictive-analytics-andlink-analysis.

76. See, e.g., Jill Wechsler, FDAAA Empowers FDA to Have Greater Control Over Drug Safety, FORMULARY WATCH (Dec. 1, 2007), http://formularyjournal.modernmedicine.com/formularyjournal/news/clinical/clinical-pharmacology/fdaaa-empowers-fda-have-greater-control-over-d (discussing FDAAA mandating enhanced communication between pre- and post-market review staff members and bi-annual reporting).

77. See Janus, U.S. FOOD \& http://www.fda.gov/ForIndustry/DataStandards/StudyDataStandards/ucm155327.htm (last updated Mar. 17,2017 ) ("The Janus is a data repository for subject-level clinical and nonclinical study data submitted to FDA as part of a regulatory submissions.").

78. See U.S. FOOD \& DRUG ADMIN., The Sentinel Initiative, http://www.fda.gov/safety/FDAsScntinelInitiative/ucm2007250.htm (last updated Nov. 21, 2017); see also Steven Findlay, Health Policy Brief: The FDA's Sentinel Initiative, HealTh AfF. BLog (June 4, 2015), http://hcalthaffairs.org/healthpolicybriefs/brief_pdfs/healthpolicybrief_139.pdf. 
authorities are increasingly likely to use big data for disease modeling, ${ }^{79}$ epidemic prediction ${ }^{80}$ and pharmacovigilance. ${ }^{81}$

Private healthcare entities "have used big data to predict life expectancy, genetic predisposition to disease, likelihood of hospital readmission, and likelihood of adherence to a treatment plan in order to tailor medical treatment to an individual's characteristics." 82 For example, some technology companies use big data techniques to decrease readmissions and improve outcomes for its customer hospitals. ${ }^{83}$ Similarly, other companies tackle readmissions by analyzing patient-specific data from admission, discharge, follow-up calls, postdischarge patient interactions, and other clinical data. ${ }^{84}$ Many health data technologies and their analytic implementations remain in the world of "small data." Even if the data are "big," many of the analytics remains at a low level (descriptive analytics or "what was it that happened?"). ${ }^{85}$. However, this is" changing and it is reasonable to assume that big data should -improve "personalization of care, assessment of quality and value for many more conditions" as well as helping "providers better manage population health and risk-based reimbursement approaches." 86

79. See, e.g., Steve Lohr, Sizing Up Big Data, Broadening Beyond the Internet, N.Y. TIMES (June 19, 2013, 11:09 PM),http://bits.blogs.nytimes.com/2013/06/19/sizing-up-big-data-broadening-beyondthe-internet/(explaining how exploration of genetic and other medical data could lead to some breakthroughs in disease modeling and treatment).

80. See, e.g., Jeremy Ginsberg et al., Detecting Influenza Epidemics Using Search Engine Query Data, 457 NATURE 1012, 1012-13 (2009) (cxplaining how monitoring google search queries can help track illncsses).

81. See generally Ryen W. White et al., Web-Scale Pharmacovigilance: Listening to Signals from the Crowd, 20 J. AM. MED. INFORMATICS ASS'N 404, 404-08 (2013).

82. Fed. TRade COMM'N, Big Data: A TOOL For InClusion OR Exclusion? 7 (2016), https:/www.ftc.gov/system/files/documents/reports/big-data-tool-inclusion-or-exclusion- - * understandingissues/160106big-data-rpt.pdf.

83. Crimson Quality Reporting, ADVISORY BOARD, https://www,advisory.com/technology/crimsonquality-reporting (last visited Sept. 9, 2017); see, e.g., Anna Wilde Mathews, Hospitals Prescribe Big Data to Track Doctors at Work, WALL ST. J. (July 11, 2013), https:/www.wsj.com/articles/hospitalsprescribe-big-data-to-track-doctors-at-work-1377188043?tesla=y (discussing how length of inpatient stays fell $2.9 \%$ and readmissions fell $4.5 \%$ ); see also Sarah Needlcman, Rx to Avoid Health-Law Fines, WALL ST. J. (Aug. 7, 2013, 7:20 PM), https://www.wsj.com/articles/rx-to-avoid-healthlaw-fines1375917632 (discussing how Medicare readmissions has dropped 1.25\%).

" 84. See, e.g., CÖORDINATED CARE, https://www.patientsafesolutions.com/platform/coordinated'care/ (last visited Feb. 20, 2018).'

85. See Tom Sullivan, Big Data: Hardest Part of Population Health and Precision Medicine?, HEALTHCARE IT NEwS (Mar. 28, 2016, 11:09 AM), http:/www.healthcareitnews.com/ncws/big-datahardest-part-population-health-and-precision-medicine ("I haven't seen big healthcare data. I've only seen little healthcare data.").

86. Robert Kocher \& Bryan Roberts, Meaningful Use of Health IT Stage 2: The Broader Meaning, HEALTH AFF. BLOG (Mar. 15, 2012), http:/healthaffairs.org/blog/2012/03/15/meaningful-use-of-healthit-stage-2-the-broader-meaning/. 


\section{Robotics}

Simply put, robots are machines that use data and computational power to help them interact with the physical world. To be robotic is to perform repetitive tasks. Robots are everywhere. They may be as inconsequential as the Roomba that sweeps your floor ${ }^{87}$ and occasionally entertains your household pets ${ }^{88}$ or as frightening as autonomous drones being tested by the Pentagon. ${ }^{89}$ There are innumerable types of robots, including swarm robots and tactile robotics. ${ }^{90}$ However, popular culture tends to concentrate on a narrow subset-humanoid robots, whether Sonny in $I$, Robot, ${ }^{91}$ the Synths in Humans, ${ }^{92}$ or the Hosts in Westworld..$^{93}$

Ironically, the most frequently identified healthcare "robot" barely fits that description. The da Vinci Surgical System ${ }^{94}$ is not (at least, not yet) autonomous but an example of teleoperation. It merely (if impressively) translates live human interactions to perform minimally-invasive micro surgery. However, there are plenty of "real" robots already working in the health domain, such as disinfectant robots, ${ }^{95}$ service robots such as the autonomous vehicles that pull laundry and

87. iRobot Roomba 980, IROBOT, https://store.irobot.com/default/roomba-vacuuming-robotvacuum-irobot-roomba-980/R980020.html (last visited Sept. 9, 2017).

88. See, e.g., Listen to That Engine Purr! Cat Hitches a Ride on a Customized Roomba Complete with a Box, Cushion and,Blanket, DAILY MAIL (Sept. 9, 2016, 7:24 PM) http://www.dailymail.co.uk/news/article-3782539/Feline-hitches-ride-customized-Roomba-turned-catcar.html (describing a viral video where a cat rides a Roomba, "snuggled inside a plastic box fitted with a blanket and cushion, and adorned with a sticker that read "cat in a car"').

89. See Matthew Rosenberg \& John Markoff, The Pentagon's 'Terminator Conundrum': Robots

That Could Kill on Their Own, N.Y. Times (Oct. 25, 2016), https://www.nytimes.com/2016/10/26/us/pentagon-artificial-intelligenceterminator.html (describing how artificial intelligence has replaced man and machine on the battlefield).

90. See Zach Weinersmith and Kelly Weinersmith, Buildings of the Future Might be Constructed by Swarms of Robots, POPULAR SCI. (Oct. 27, 2017), https://www.popsci.com/buildings-future-might-beconstructed-by-swarms-robots (describing how a large amount of small robots could construct a house); see Tactile Robotics, BRISTOL ROBOTIC LABORATORY, http://www.brl.ac.uk/research/researchthemes/tactilcrobotics.aspx (last updated Dec. 10, 2016) (explaining tactile robotics as the development of artificial touch).

91. See Sonny (Character), IMDB, http:/www.imdb.com/character/ch0005853/?ref_=tt_cl_t3 (last visited Sept. 6, 2017); see also Russell Scott Smith, Real Man of Steel-Meet Sonny, The Star of 'I-Robot'And Maybe Our Future, N.Y. PosT (July 11, 2014, 4:00 AM), https://nypost.com/2004/07/1 1/real-manof-steel-meet-sonny-the-star-of-irobot-and-maybe-our-future/ (describing Sonny, a "highly advanced prototype robot").

92. Humans: About the Show, AMC, http://www.amc.com/shows/humans/cxclusives/about (last visited Nov. 18, 2017) (describing "Synths," synthetic humanoid robot characters in the television show).

93. See Megan Eisenfelder, Westworld: Are the Hosts Human?, Grounds (Jan. 3, 2017), http://www.vabioethics.com/content/2017/1/3/westworld-are-the-hosts-human (explaining the "Hosts," humanoid robots in the television show).

94. DA VINCI SURGERY, http://www.davincisurgery.com (last visited Sept. 6, 2017).

95. See, e.g., Why Choose Xenex?, XENEX, http://xenex.com/about-xenex (last visited Sept. 6, 2017) (explaining that the Pulsed Xenon Full Spectrum UV system is an example of a disinfectant robot). 
pharmaceutical carts around hospitals, ${ }^{96}$ and automated pharmacy systems that eliminate human "picking" errors. ${ }^{97}$

In the words of Ryan Calo, "Robots combine, arguably for the first time, the generative promiscuity of data with the capacity to do physical harm." 98 Primitive healthcare robots already cause physical harms ${ }^{99}$ and have the attention of regulators. ${ }^{100}$ These are issues that are within the purview of both the original and new triangles. It is the subtler uses of robots in the healthcare environment (and here Calo's discussion of embodiment is on point ${ }^{101}$ ) that will be more challenging. Outside of healthcare facilities robots and their integration with other monitoring technologies are viewed as a major solution to the caregiving problems faced by countries such as Japan and the United States because of ageing populations. ${ }^{102}$ Increasingly these robots will provide medication assistance, move patients, and once part of the care team,- inevitably communicate with human professionals. ${ }^{103}$

One dystopian proof-of-concept posted online demonstrates not only the healthcare role that a comfort robot can fill but also the profound social change that artificial empathy suggests. The "End of Life Care Machine" comforts a person in the last few minutes of her life, stroking her arm while delivering chilling platitudes such as "I am sorry that your family and friends cannot be

96. See, e.g., TUG Robots - Healthcare Benefits, AETHON, http://www.acthon.com/tug/tughealthcare (last visited Sept. 6, 2017); sec also Matt Simon, Meet Tug, the Busy Little Robot Nurse Will See You Now, WIRED (Nov. 10, 2017, 8:00 AM), https://www.wired.com/story/tug-the-busy-little-robot-nursewill-see-you-now/ ("[I]n hospitals around the world, this robot is helping nurses and doctors care for patients by autonomously delivering food and drugs[.]").

97. James ManyiKa et al., Mckinsey Global INSt., A Future That Works: Automation, EMPLOYMENT, AND PRODUCTIVITY . 23 (2017), http://www.mckinsey.com/ /media/McKinsey/Global\%20Themes/Digital\%20Disruption/Harnessing\%2 0automation $\% 20$ for $\% 20 \mathrm{a} \% 20$ future $\% 20$ that $\% 20$ works/MGI-A-future-that-works_Full-report.ashx.

98. Ryan Calo, Robotics and the Lessons of Cyberlaw, 103 CAL. L. REv. 513, 534 (2015).

99. See, e.g., Homa Alemzadeh et al., Adverse Events in Robotic Surgery: A Retrospective Study of 14 Years of FDA Data, PLOS ONE 1, 8 (2016) (describing that there were 58 deaths, 938 patient injuries, and 4,124 malfunctions in 2013).

100. See, e.g., Warning Letter from Elizabeth A. Kage, Acting Dist. Dir., Food \& Drug Admin., to Gary S. Guthart, President \& CEO, Intuitive Surgical Inc. (July 16, 2013), https:/www.fda.gov/iceci/enforcementactions/warningletters/2013/ucm 363260 htm (indicating that inspection revealed that robots are misbranded).

101. See Calo, supra note 98 , at 532-37.

102. See Christian Crisostomo, Robots: Japan's Future Elderly Care Workers, VRWORLD (Jan. 22, 2015), https://vrworld.com/2015/01/22/robots-japans-future-elderly-care-workers/ (reporting that Japan has begun developing robots for its elderly population); see also Gabriella Mulligan, Is Robotics a Solution to the Growing Needs of the Elderly? BBC NEws (Mar. 17, 2017), http:/www.bbc.com/news/business39255244 ? (stating that U.S. technology company, IBM, is performing research on the idea of robotic nurses to provide quality care for the elderly).

103. Drew Simshaw et al., Regulating Healthcare Robots: Maximizing Opportunities While Minimizing Risks, 22 RICH. J.L. \& TECH., no. 3, 2016, at 10-11. 
with you right now, but don't be afraid, I am here to comfort you" and "You are not alone, you are with me."104

So far, few healthcare robots have adopted human-like form factors. However, that design ethos will likely develop from existing companion robots $^{105}$ and many robots will become considerably more humanoid in their shape and affect. ${ }^{106}$ However, "real" healthcare robots are unlikely to be humanoid. Their shape will follow their function. A good, but as discussed later, ${ }^{107}$ controversial example is the Sedasys anesthesiology machine. This FDA approved $^{108}$ device applies moderate sedation for procedures such as colonoscopies using the drug Propofol and is monitored by nurse,s not anesthesiologists. ${ }^{109}$ No child asked to draw a picture of a robot would come up with anything resembling such a functional design. However, machines that combine real-world interaction with $\mathrm{AI} /$ Machine Learning will likely make up the majority of healthcare "robots." Whether we end up talking about healthcare robots or healthcare machine learning likely will be a function of whether the product of the AI will find physical expression through a machine or a person.

\section{Artificial Intelligence and Machine Learning}

The relationship between Data Analytics, AI, and Machine Learning is complex. In essence, they form something of a data technology turducken! Diagnostic, predictive, and prescriptive analytics increasingly are powered by $\mathrm{AI}$, and currently the most powerful type AI flavor features Machine Learning. ${ }^{110}$ As Bernard Marr notes, given the power of computing delivered by Moore's Law and the almost limitless amount of data available on the Internet, "engineers realized that rather than teaching computers and machines how to do everything,

104. End of Life Care Machine, DAN CHEN, http://www.pixedge.com/lastmoment (last visited Sept. $9,2017)$.

105. See, e.g., PARO Therapeutic Robot, PARO, http://www.parorobots.com (last visited Sept. 9, 2017) (explaining that the PARO Therapeutic Robot can learn to behave in a way that users prefer and explains how it relates to animal therapy).

106. See Joe Bush, Widening the Appeal of Humanoid Robots, ELEC. SPECIFIER (Apr. 21, 2016), http:/www.electronicspecifier.com/robotics/widening-the-appeal-of-humanoid-robots (explaining the capabilitics of of ZoraBots and how they provide companionship benefits); see, e.g., Asha McLcan, CES 2017: Panasonic Unveils 'Human-Like' Companion Robot, ZDNET (Jan. 5, 2017, 3:24 PM), http:/www.zdnet.com/article/ces-2017-panasonic-unveils-human-like-companion-robot/ (explaining how the robots will have human-like capabilities such as vision, navigation, and motion control skills).

107. See infra note 188 (explaining that the Sedasys machine was created to "sedate patients for routine medical procedures").

108. U.S. FOOD \& DRUG ADMIN, PREMARKET APPROVAL (PMA), https://www.accessdata.fda.gov/scripts/cdrh/cfdocs/cfpma/pma.cfm?id=p080009 (last updated Sept. 4, 2017).

109. Tom Simonite, Automated Anesthesiologist Suffers a Painful Defeat, MIT TECH. REV. (Mar. 29, 2016), https://www.technologyrevicw.com/s/601141/automated-anesthesiologist-suffers-a-painfuldefeat/ (explaining that the machine will only require a nurse, not a doctor).

110. See generally Tom Simonite, The Wired Guide to Artificial Intelligence, WIRED (Feb. 01, 2018). 
it would be far more efficient to code them to think like human beings, and then plug them into the internet to give them access to all of the information in the world." 11 According to Brynjolfsson and McAfee, "[T]he exponential, digital, and recombinant powers of the second machine age have made it possible for humanity to create two of the most important one-time events in our history," AI and "a common digital network." 12

The shift from information technologies and personal or business computing to data technologies, algorithms, and machine learning may appear to be progressive, the running of a steady continuum of technological progress. This is unlikely to be the case. Machine Learning, and hence AI, is developing very quickly in large part because programmers are making neural networks that essentially mimic some of the ways that humans think. "Dry brains" that can think like "wet" brains are pushing the technology forward at a dramatic rate.This is an area that is deeply complex. For example, Pedro Domingos talks about the "five tribes of machine learning," each with its own "master algorithm, a general-purpose learner that you can in principle use to discover knowledge from data in any domain," noting "What we really want is a single algorithm combining the key features of all of them: the ultimate master algorithm." 113 It is also an area that is developing rapidly. Recently, Google researchers reported that they had made progress in solving the "catastrophic forgetting" problem in neural networks ${ }^{114}$ such that $\mathrm{AI}$ will be able to learn tasks sequentially. ${ }^{115}$

Much of the critical analysis of AI has focused on its eçonomic impact, particularly its adverse effects on employment and the distribution of wealth. ${ }^{116}$

111. Bernard Marr, What is the Difference Between Artificial Intelligence and Machine Learning?, FORBES (Dec. 6, 2016, 2:24 AM), https://www.forbes.com/sites/bernardmarr/2016/12/06/what-is-thedifference-between-artificial-intelligence-and-machine-learning/ $\$ 4090 \mathrm{e} 19 \mathrm{e} 2742$.

112. BRYNJOLFSSON \& MCAFEE, supra note 29, at 89.

113. Pedro Domingos, The Master Algorithm: How the Quest for the Ultimate Learning MACHINE WILl REMAKE OUR WORLD, at xvii (2015).

114. See, e.g., Shelly Fan, New Artificial Synapse Bridges the Gap to Brain-Like Computers, SINGULARITYHUB (Mar, 15, 2017), https://singularityhub.com/2017/03/15/new-artificial-synapse-bridgesthe-gap-to-brain-like-computers/? (providing an example of an artificial neural network technology that is effective at making brain-like computations); see also James Kirkpatrick et al., Overcoming Catastrophic Forgetting in Neural Networks, 114 PROC. NAT'L ACAD. SCI. U.S. 3521, 3524 (2017), http://www.pnas.org/content/1 14/13/3521.full.pdf (cxplaining catastrophic forgetting as a phenomena in artificial neural networks where knowledge of a previous programmed taks is abruptly lost as a new task is incorporated).

115. See Kirkpatrick et al., supra note 114 (explaining the approach to making AI able to learn tasks sequentially in which old tasks are remembered selectively slowing.down learning on the weights important for those tasks).

116. See David Rotman, Technology and Inequality, MIT TECH. REv. (Oct. 21, 2014), https:/www.technologyreview.com/s/531726/technology-and-inequality/ (noting that Al may be the biggest factor in recent increases in economic inequality) [hereinafter Rotman, Technology and Inequality]; see also David Rotman, The Relentless Pace of Automation, MIT TECH. REv. (Fcb. 13, 2017), 
Indeed, an Obama-era White House report concluded that, at the macro level, automation would drive considerable economic growth but at the potential cost of forty-seven percent of U.S., primarily lower-skilled jobs. ${ }^{177}$ It seems within the realm of the probable that AI will have a similar impact on healthcare.

One vision of that impact is bell-shaped, with AI having the most impact on the outliers-replacing not only the least skilled manual tasks such as custodial or transportation but also tasks that require great skills or concentration that humans seldom can master. For example, when IBM's Watson is used to interpret whole genomic data it can deliver "clinically actionable insights within 10 minutes, compared to 160 hours of human analysis and curation typically required to reach similar conclusions." 18 Similarly, the FDA recently cleared cloud-based, machine learning cardiac imaging software that takes 15 seconds per case compared to the thirty minutes a human would take. ${ }^{119}$ As, Kevin Kelly argues, "While the displacement of formerly human jobs gets all the headlines, the greatest benefits bestowed by robots and automation come from their occupation of jobs we are unable to do. We don't have the attention span to inspect every square millimeter of every CAT scan looking for cancer cells." 20

A 2017 McKinsey study developed a metric based on eighteen "capability requirements" of various "work activities," grouped by "sensory perception, cognitive capabilities, natural language processing, social and emotional capabilities, and physical capabilities." 21 The study concluded "While less than 5 percent of occupations can be fully automated, about 60 percent have at least 30 percent of activities that can technically be automated"122 Work activities that were more susceptible to automation involved predictable physical activities,

https:/www.technologyreview.com/s/603465/the-relentless-pace-of-automation/ (suggesting that automation may improve the economy at the cost of jobs and increases in income inequality) [hereinafter Rotman, The Relentless Pace of Automation].

117. Exec. OfFice of the President, Artificial intelligence, Automation, and the $\begin{array}{lllll}\text { ECONOMY } & 2, & 8, & 13 & \text { (2016), }\end{array}$ https:/obamawhitehouse.archives.gov/sites/whitchouse.gov/files/documents/Artificial-IntelligenceAutomation-Economy.PDF.

118. Bernic Monegain, AI Can Speed up Precision Medicine, New York Genome Center-IBM Watson Study Shows, HEALTHCARE IT NEwS (July 14, 2017, 1:15 PM), http:/www.healthcareitnews.com/news/ai-can-speed-precision-medicine-new-york-genome-center-ibmwatson-study-shows.

119. Bernard Marr, First FDA Approval for Clinical Cloud-Based Deep Learning in Healthcare, FORBES (Jan. 20, 2017, 2:11 AM), https://www.forbes.com/sites/bernardmarr/2017/01/20/first-fdaapproval-for-clinical-cloud-based-deep-learning-in-healthcare/.

120. Kelly, supra note 25.

121. MANYIKA ET AL., supra note 97, at 4.

122. Id. at 5 exhibit $\mathrm{E} 2$. 
data collection and data processing, ${ }^{123}$ generally jobs that are commodified. ${ }^{124}$ In contrast, current automation technologies had relatively low scores on social and emotional capabilities, such as sensing emotional states (inputs) or displaying empathy (outputs). ${ }^{125}$ The study scored healthcare and professionals generally as having relatively lower potential for automation. Overall, jobs that value creativity, complex relationships, or involve unpredictable tasks or locations are least likely to be automated. ${ }^{126}$ As importantly, a healthcare case study McKinsey performed suggested that most of the gains would be in labor substitution with only a relatively small performance gain. ${ }^{127}$ The conclusions seem broadly consistent with a hypothesis in the White House report that while "AI technology ... may improve early detection of some cancers or other illnesses," nevertheless it will take a human "to work with patients to understand and translate patients' symptoms, inform patients"of-treatment options, and guide patients through treatment plans." 128 .

\section{Technology Typology and Properties}

Automation's present and future healthcare data technologies (Apps, IoT, Robotics, and AI) are closely related. However, their properties are not rectilinear, although some through lines are identifiable based on function. For example, some of these technologies primarily collect data, such as smartphones, wearables, IoT, and data-miners. Both smartphones and IoT provide real-world contextualization. However, data analytics and AI tend to be more about processing data (many smartphones still process data but increasingly will offload these tasks to cloud-based AI).

These technologies do share some other, more conceptual properties. First, generally they produce nonrival goods. Marcus Welby, MD probably saw eight patients a day. However, the algorithms of the neural networks located in the

123. Id. at 6 exhibit E3.

124. See generally Thomas H. Davenport, When Jobs Become Commodities, MITSLOAN MGMT. REV. (July 21, 2017), http://sloanreview.mit.cdu/article/when-jobs-become-commodities/amp/ ("Simply put, if a job is viewed as a commodity, it won't be long before it is automated.").

125. See MANYIKA ET AL., supra note 97, at 35 exhibit 3 (illustrates that current automation performance is below median for social and emotional capabilities in comparison to human performance).

126. See Arwa Mahdawi, What Jobs Will Still Be Around in 20 Years?, GuARdian (June 26, 2017, 6:00 AM), https:/www.theguardian.com/us-news/2017/jun/26/jobs-future-automation-robots-skillscreative-health.

127. See MANYIKA ET AL., supra note 97, at 35 exhibit 3 (suggesting technology is above median capability for gross motor skills in comparison to human performance).

128. Exec. Office of the President, ARtificial Intelligence, Automation, and the ECONOMY 18

(2016),

https://obamawhitehouse.archives.gov/sites/whitehouse.gov/files/documents/Artificial-IntelligenceAutomation-Economy.PDF. 
cloud-enabling solar-powered server farm in Moncks Corner, South Carolina ${ }^{129}$ could "see" 100 , or a thousand, or a million patients at the same time. ${ }^{130}$ As a result, the impact of noncompeting technologies on the Access point of the original triangle could be substantial.

Second, the data being collected and processed by these technologies go far beyond the clinical data used to treat individual patients or even the predictive personalized data that will profile those individuals and their likely future health. The data will also address "the health of populations," part of the Triple Aim ${ }^{131}$ discussed below. ${ }^{132}$ The population and public health potential of these data troves and greater understanding of the health of the whole "herd" should be significant. $^{133}$ Of course, what happens with that data will depend on policymakers' interest in preventative care and the extent to which they believe that herd-health impacts individual health.

Third, these data technologies increasingly operate outside of what for a century we have understood as our healthcare system, a system dominated by bricks-and-mortar, professional hegemony, and until quite recently, paper files. The health information technologies such as EMRs and clinical decision support systems that emerged at the end of the twentieth century were part of the incumbents' clinical, domain and so had little disruptive effect. ${ }^{134}$ However, Clay Christensen's disruption theory is far more relevant to the health data technologies considered here. These are not sustaining technologies owned by incumbents but disruptive ones controlled by potential attackers. ${ }^{135}$ Increasingly, data collection is performed by patients or pre-patients, IoT sensors or by big data brokers leveraging medically-inflected data. The disruption could be quite significant. Assuming that healthcare providers will be slow to transform themselves into data companies, it is likely that data companies with massive scale and cloud-based storage and processing will aggregate and process future

129. Berkeley South Carolina Datacenter, DATACENTERS.COM, https://www.datacenters.com/googleberkelcy-south-carolina-2 (last visited Dec. 22, 2017).

130. I concede the oversimplification here. For example, hardware or personnel required at some of the bricks-and-mortar nodes will be rival. See Artificial Intelligence Will Redesign Healthcare, MEDICAL FUTURIST INST., http://medicalfuturist.com/artificial-intelligence-will-redesign-healthcare/ (last visited Nov. 17, 2017) (discussing the "efficiency of diagnosing patients can increase by multiple times" that can be accomplished with artificial intelligence applications in comparison to traditional health examinations).

131. Donald M. Berwick et al., The Triple Aim: Care, Health, and Cost, 27 Health AfF. BLog. 759, 768 (2008), http://www.healthaffairs.org/doi/pdf/10.1377/hlthaff.27.3.759.

132. See infra text accompanying notes $358-61$.

133. See Tae Hyong Kim et al., Vaccine Herd Effect, 43 SCANDINAVIAN J. INFECTIOUS DiSEASES 683 , 688 (2011) (explaining that the "herd effect" gives benefits to a population beyond the original "targeted population").

134. See generally Terry, supra note 41 (explaining that information technology is underutilized in healthcare).

135. See generally Clayton M. Christensen, The Innovator's Dilemma: When New TECHNOLOGIES CAUSE GREAT FiRMS To FAil 55 (Tushman et al. eds., 2016) (cxplaining that entrance firms have an "attacker's advantage" in innovation). 
health data. Pre-patients and patients will be both consumers of data services and the nodes that collect much of the data. Patients will become more like automobiles whose computers announce when they have a fault or require routine maintenance. A healthcare system that has struggled using payment reform to change from services pushed by providers to those pulled by patients will face an entirely new paradigm; algorithms determining what services are to be offered based on patient condition, cost-effectiveness of procedure, insurance status, patient compliance, etc. A particularly interesting question will be the identity of the healthcare service providers in this new world. Will they not only be the customers of the data companies but also their subsidiaries? ${ }^{136}$

Fourth, AI and its increasingly massive number of nodes (phones, IOT, etc.) benefit from a phenomenon similar to the network effect. ${ }^{137}$ These technologies and the products built on top of them will continually and exponentially improve: As AI harvests more data it will also learn more about its own performance and through a feedback loop instruct the nodes about improving their data collection. AI health data analysis likely will increase in volume and accuracy at a rate unknown for traditional healthcare analyses such as outcomes research. ${ }^{138}$

\section{VALUE}

As already noted the Value point of the new triangle includes quality, safety, effectiveness, efficiency and data protection. Most of these inclusions should be unsurprising given the many reports of quality, safety, and data protection flaws inherent in health data technologies. The addition of efficiency and effectiveness considerations may seem less obvious: The policy choice is to subject Automation to cost-effectiveness analysis. (CEA), or comparative effectiveness research (CER) such that new healthcare data technologies are not adopted solely because the technology is available but that they provide Value as determined by the (Quality + Outcomes) / Cost formulä.

This approach is not particularly novel. Its roots are visible in some common-law concepts, notably the Hand formula. ${ }^{139}$ Further, for decades, the

136. See generally Ben Thompson, Amażon's New Customer, STRATECHERY (June 19, 2017), https://stratechery.com/2017/amazons-new-customer/ (comparing Amazon's new buying of Whole Foods).

137. See generally Alex Housley \& Santiago Tenorio, AI Innovation Will Trigger the Robotics Network Effect, VentureBEAT (Nov. 29, 2017) (explaining that the network effect..."s a dynamic in which increased use of a service actually improves the service, such as how machine-learning models generally grow more accurate as a result of training from larger and larger volumes of data.").

138. See generally The World's Most Valuable Resource Is No Longer Oil, but Data, ECONOMIST (May 6, 2017), http://www.economist.com/news/leaders/21721656-data-economy-demands-newapproach-antitrust-rules-worlds-most-valuable-resource? . ("Meanwhile, artificial-intelligence (AI) techniques such as machine learning extract more value from data.").

139. See generally United States v. Carroll Towing, 159 F.2d 169, 173 (1947) (discussing liability for injuries such that liability depends on whether the burden is less than the injury multiplied by probability); see Kenneth W. Simons, The Hand Formula in the Draft Restatement, 54 VAND. L. REV. 901, 903 (2001) 
approach to determining whether a product is defective (aka unsafe) in products liability cases has been to instruct juries on risks vs benefits. ${ }^{140}$ Essentially, the courts use CER, instructing juries to compare a plaintiff's contended-for substitute design with the manufacturer's original design. Similar reasoning also underpins the well-known case of Helling $v$. Carey; ${ }^{141}$ plaintiff there used costbenefit analysis as a substitute for custom in a medical malpractice case. More recently, courts and scholars have raised the question of the appropriate use of clinical practice guidelines (that are based on CER or $\mathrm{CEA}^{142}$ ) in setting the liability standard in medical malpractice cases. ${ }^{143}$

Value has been less successful in establishing explicit adoption in the health regulatory space. Although historically the FDA has only used effectiveness (in the sense of efficacy) in drug and device assessment, increasingly the agency acknowledges a "risk-based" approach to approvals. ${ }^{144}$ Any hesitancy regarding the broad adoption of CEA in healthcare is likely attributable to its core metric of the quality-adjusted life year (QALY) that is routinely used by other

(stating that "'risk-bencfit,' 'cost-benefit,' and 'balancing' are all acceptable descriptions because they all express the "simple idea' that '[c]onduct is negligent if its disadvantages exceed its advantages" in characterizing the Hand formula) (internal citation omitted).

140. See, e.g., Barker v. Lull Engineering Co., Inc., 573 P.2d 443, 446 (Cal. 1978) (explaining that "when a product is claimed to be defective because of an unsafe design or an inadequate warning, the contours of the defect concept may not be self-evident"); see also RESTATEMENT (THIRD) OF TORTS: PRODUCTS LIABILITY § 2(b) (AM. LAW INST. 1998) ("A product... is defective in design when the foreseeable risks of harm posed by the product could have been reduced or avoided by the adoption of a reasonable alternative design by the seller or other distributor, or a predecessor in the commercial chain of distribution, and the omission of the alternative design renders the product not reasonably safe[.]").

141. 519 P.2d 981, 982 (Wash. 1974) (explaining that although the defendant physicians were compliant with the standard of care in treatment, the benefit of performing a routine pressure test outweighed the risk of the patient's loss of vision).

142. See, e.g., Jeffrcy L. Anderson et al., ACC/AHA Statement on Cost/Value Methodology in Clinical Practice Guidelines and Performance Measures, 22 AM. HEART ASs'N J. 2329, 2335 (2014) (explaining the key concept of cost-effectiveness "is that the value of an intervention or a program must be considered in incremental terms compared with the relevant alternatives.")

143. See, e.g., Hinlicky v. Dreyfuss, 848 N.E.2d 1285, 1290 (N.Y. 2006) (court discusses whether "clinical practice guidelines" should be admissible as "cvidence of the standard of care" for medical liability); see also Michelle M. Mello, Of Swords and Shields: The Role of Clinical Practice Guidelines in Medical Malpractice Litigation, 149 U. PA. L. REV. 645, 647 (2001) ("Because they derive from the consensus of experts, CPGs are thought to represent the prevailing standard of care in the medical profession.").

144. See U.S. Food \& Drug Admin., Pharmaceutical cGMPS for the $21^{\text {st }}$ Century-A RiskBASED APPROACH: SECOND PROGRESS REPORT AND IMPLEMENTATION PLAN (2015), https://www.fda.gov/Drugs/DevelopmentApprovalProcess/Manufacturing/QuestionsandAnswersonCurr entGoodManufacturingPracticescGMPforDrugs/UCM071836 (explanatory parenthetical?); see also Scott Gottlieb, Fostering Medical Innovation: A Plan for Digital Health Devices, FDA VolCE (June 15, 2017), https://blogs.fda.gov/fdavoice/index.php/2017/06/fostering-medical-innovation-a-plan-for-digitalhealth-devices/. 
technology assessment agencies, ${ }^{145}$ but which is politically toxic in the U.S. ${ }^{146}$ This may change as value becomes a touchstone for meaningful healthcare cost containment though programs such as Hospital Value-Based Purchasing ${ }^{147}$ and the MACRA Quality Payment Program. ${ }^{148}$

The IOM Quality Domains (and hence my conception of Value) include effectiveness, efficiency, equity, patient centeredness, safety, and timeliness. ${ }^{149}$ I both subtract and add properties to Value: For example, I take the position that equity and patient centeredness are more appropriately positioned under Empathy. On the other hand, I add data protection (including data quality) to Value. Finally, I prefer the term cost-effectiveness to the concepts of effectiveness and efficiency.

The broad range of Value can be illustrated with some real-world cases.

- In early 2016, a patient was undergoing cardiac catheterization in a hospital cath lab. The lab used a real-time monitoring module connected to a PC. During the catheterization, the monitor suddenly went black and the patient has to be sedated while the computer was rebooted. The reason? The computer had automatically launched an anti-malware scan. After the reboot the procedure was successfully completed. ${ }^{150}$

- The infamous Target stores case was first reported by Charles Duhigg. He explained how Target Corp. used predictive analytics to identify potential customers in their second trimester of pregnancy so that they could send them targeted advertising. This was achieved primarily by using analytics to discover changes in

145. See, e.g., Michael D. Rawlins, NICE: Moving Onward, 369 NEw ENG. J. MED. 3, 4 (2013) ("Priorities must be set on the basis of evidence of both clinical effectiveness and cost-effectiveness. However, priority setting in health care must encompass more than the technical and scientific demands of health technology assessment.")

146. See, e.g., Peter J. Neumann \& Milton C. Weinstein, Legislating Against Use of Cost-Effectiveness Information, 363 NEW ENG. J. MED. 1495, 1496 (2010) ("At a time when health care costs loom as the greatest challenge facing our country's fiscal well-being, legislating against the use of the standard metric in the field of cost-effectiveness analysis is regrettable.").

. 147. Hospital Value-Based Purchasing (VBP) Program, CTRS. FOR MEdICARE \& MEdICAID SERVS., https://www.cms.gov/Medicare/Quality-Initiatives-Patient-Assessment-Instruments/Valie-Based-

Programs/HVBP/Hospital-Value-Based-Purchasing.html (last modified Jan: 11, 2017).

148. What's MACRA?, supra note 15.

149. Understanding Quality Measurement, AHRQ, https://www.ahrq.gov/professionals/qualitypatient-safety/quality-resources/tools/chtoolbx/understand/index.html (last reviewed Nov. 2017).

150. MAUDE Adverse Event Report: Merge Healthcare Merge Hemo Programmable Diagnostic Computer, U.S. FOOD \& DRUG . ADMIN., https:/www.accessdata.fda.gov/scripts/cdrh/cfdocs/cfmaude/detail.cfm?mdrfoi- id =5487204 (last " updated Nov. 30, 2017); see also Dan Goodin, That Time a Patient's Heart Procedure Was Interrupted by $a$ Virus Scan, ARs. TECHNICA (May 16, 2016, 1:58PM), https://arstechnica.com/security/2016/05/faulty-av-scan-disrupts-patients-heart-procedure-whenmonitor-goes-black/. 
the patterns of product purchases (e.g., changing to unscented lotion). Target then sent coupons for baby clothes and cribs to those identified, leading to angry complaints from the father of a teenage recipient. A few days later the father spoke with a Target manager again, "It turns out there's been some activities in my house I haven't been completely aware of. She's due in August." 151

- On October $21^{\text {st }} 2016$, there was widespread disruption of Internet services in the US. The cause was a series of Distributed Denial of Service (DDoS) attacks. These particular DDoS attacks were unique because the bot net used was not constructed of infected PCs. Rather, it used malware-infected members of the Internet of Things, primarily unsecured routers, connected cameras, and DVRs. Frequently, these devices are still using their default security settings and are extremely soft targets. ${ }^{152}$

- The adoption of interoperable EMRs have long been touted as an effective strategy for the improvement of healthcare across broad quality, safety, and cost-containment metrics. However, then strong policy arguments in favor of adoption proved unable to overcome some classic market failures and the prevalence of underperforming products. The response of the federal government was to turn to a subsidy model, CMS's "meaningful use" program introduced by the HITECH Act of 2009. ${ }^{153}$ However, neither the meaningful use criteria nor its certification hardware-software corollary delivered cost-effective EMRs. Rather, the devices have been broadly criticized for their ineffective interfaces, their empathy-sapping interference with the physician-patient relationship, and other quality and safety lapses. ${ }^{154}$

151. Charles Duhigg, How Companies Learn Your Secrets, N.Y. TIMES (Feb. 16, 2012), http://www.nytimes.com/2012/02/19/magazine/shopping-habits.html?.

152. See Stephen Cobb, 10 Things to Know About the October 21 IoT DDos Attacks, WELIVESECURITY (Oct. 24, 2016, 07:16 PM), https://www.welivesecurity.com/2016/10/24/10-thingsknow-october-21-iot-ddos-attacks/ ("Default usernames and passwords for most devices can easily be found on the Internet, making devices with default passwords extremely vulnerable."); see also Eric Limer, How Hackers Wrecked the Internet Using DVRs and Webcams, POPULAR MECHANICS (Oct. 21, 2016), http://www.popularmechanics.com/technology/infrastructure/a23504/mirai-botnet-internet-ofthings-ddos-attack/ ("Designed to target the Internet of Things specifically, Mirai can scoop up connected devices and add them to a botnet simply by attempting to log into them with their factory-default username and password.").

153. Health Information Technology for Economic and Clinical Health Act (HITECH), Pub. L. No. $111-5, \S 4101,123$ Stat. 115, 469-70 (codified at 42 U.S.C. $\S 1395 w-4(0)(2)$ (2016)) (stating that meaningful use of certified EHR technology must be certified by the Secretary of the Department of Health and Human Services).

154. See generally Nicolas P. Terry, Meaningful Adoption: What We Know or Think We Know about the Financing, Effectiveness, Quality, and Safety of Electronic Medical Records, 34 J. LEGAL MED. 7, 32 
- Online app stores increasingly sell physical improvement apps. One broadly available app is Ultimeyes that featured visual exercises related to reading speed, contrast sensitivity and low light conditions. The developer claimed the app "[i]mprove[d] vision on average by $31 \%$ and two lines on the Snellen eye chart, and improves contrast sensitivity by $100 \%$. . [and] [r]everse[d], delay[ed], or correct[ed] aging eye or presbyopia." The FTC successfully charged the marketers with making deceptive claims. ${ }^{155}$ Now in the app store the Ultimeyes value proposition has been restated as "just follow the on-screen prompts and complete four 25-minute sessions per week for a total of eight weeks. Although results vary from person to person many ULTIMEYES $®$ users see their 'vision score-in ULTIMEYES $B$ improve in a few weeks." 156 .

This section suggests broad conceptions of Value and how they are regulated, outlines how health data technologies are regulated today and in the near future, and highlights some areas of over-regulation and under-regulation.

\section{A. Regulation of Value}

Value, particularly through its elements of safety, quality, and data protection properties, is frequently operationalized by regulation. Regulatory models largely play out as hard law. Thus, healthcare data technologies may be subject to legislation, formal notice and comment regulation, (softer) subregulatory guidance, agency determinations (e.g. emanating from FTC or OCR), or judicial regulation (e.g., common law principles). A few "regulatory" models will also be the function of private ordering (e.g., accreditation), curation (e.g., by providers or app stores), or market threats (e.g., the exercise case of guild power).

The Economist, in a discussion of robot regulation has stated, "overly rigid regulations might stifle innovation. But a lack of legal clarity leaves devicemakers, doctors, patients and insurers in the dark." ${ }^{57}$ Nathan Cortez, a prominent law professor, splits from the conventional approach that favors a light regulatory touch during a time of technological innovation. Rather, he has argued, "agencies need not be so deliberate and tentative with regulating innovations-even

(2013) (noting that although some issues may resolve over time, empirical studies support persistent usability issues in health information technology).

155. FTC Approves Final Order Prohibiting "Ultimeyes" Manufacturer from Making Deceptive Claims That the App Can Improve Users' Vision, Fed. TRADE COMM'N (Feb. 23, 2016), https://www.ftc.gov/news-events/press-releases/2016/02/ftc-approves-final-order-prohibiting-ultimeyesmanufacturer.

156. Complaint at 4, In the Matter of Carrot Neurotechnolgy, Inc. (2015) (No. 142 3132).

157. You, Robot?, ECONOMIST (Sept. 1, 2012), http:/www.economist.com/node/21560986 (quoting Noel Sharkey, a computer scientist at the University of Sheffield). 
disruptive ones [and] [t]he public interest demands that agencies maintain their fortitude in the face of regulatory disruption. And, somewhat counterintuitively, new technologies can benefit from decisive, well-timed regulation." 158

There should be a premarket review of healthcare that avoids current regulatory lacunae between FDA, FTC, and HHS-OCR to mitigate some of the timing issues. Robots should be subject to a foundational regulatory framework that will foster innovation and confidence in robotics and ensure that we maximize robotic potential in healthcare. ${ }^{159}$ Space and automotive pioneer Elon Musk has argued for a preemptive regulatory approach to $\mathrm{AI}$ : " $\mathrm{AI}$ is a rare case where we need to be proactive in regulation instead of reactive because if we're reactive in AI regulation it's too late." 160

A difficult question arises when healthcare apps, devices, or even robots are aimed at a primarily consumer rather than medical market and whether such a distinction is salient. There is little doubt that anesthesiology machines or robotic physician-extenders should be regulated as medical devices and be subject to premarket approval. However, can the same be said for carer robots sold for inhome use ${ }^{161}$ A similar dynamic exists regarding mobile apps. ${ }^{162}$ Although some hardware or software products are clearly designed for only professional use (such as providing an interface for a surgical monitoring device or controlling a medical device) or are replacements for regulated devices, ${ }^{163}$ the propagation of separate regulatory models should be resisted. Differential regimes merely tempt unscrupulous businesses to manipulate product descriptions that facilitate regulatory disruption. ${ }^{164}$ As discussed below, this professional-consumer divide is particularly troubling in the context of data protection. ${ }^{165}$ While some

158. Cortez, supra note 1 at 179-80, 227.

159. See Simshaw et al., supra note 103 , at 4 (advocating for a "foundational regulatory framework for both medical devices and consumers that is attuned to safety, security, and privacy").

160. James Titcomb, AI Is the Biggest Risk We Face as a Civilisation, Elon Musk Says, TELEGRAPH (July 17, 2017, 9:46 AM), http://www.telegraph.co.uk/technology/2017/07/17/ai-biggest-risk-facecivilisation-clon-musk-says.

161. See Simshaw et al, supra note 103, at 16-17 (indicating that care robots may begin to show autonomous or semi-autonomous characteristics).

162. See Terry, supra note 46 , at 1430 (explaining that different categories of apps call for varying safety and privacy regulations).

163. See, e.g., U.S. FoOd \& Drug Admin., Mobile Medical Applications: Guidance for INDUSTRY AND FOOD AND DRUG ADMINISTRATION STAFF 27-29 (2015) [hereinafter 2015 FDA MOBILE MEDICAL APPLICATIONS GUIDANCE]

http://www.fda.gov/downloads/MedicalDevices/DeviceRegulationandGuidance/GuidanceDocuments/U CM263366.pdf (noting that some mobile apps meet the definition of a medical device and are the focus of FDA regulatory oversight).

164. Terry, supra note 1 , at 146, 156 (stating that initial regulation disruption allows for businesses to exploit regulatory gaps and further turbulence).

165. See The Digital Divide Over Consumer Data Widens, FIn. TIMEs (July 26, 2017), https://www.ft.com/content/0799f6be-720d-11e7-aca6-c6bd07dfla3c (stating that as the rush for consumer data intensifies, technology companies face issues keeping up with privacy and data protection). 
regulatory models recognize a provider-facing consumer-facing dichotomy, any such dichotomy is based on poorly though out policy.

Some easing of FDA scrutiny of medical devices was previewed in the $21 \mathrm{st}$ Century Cures $\mathrm{Act}^{166}$ signed by President Obama. For example, review of "breakthrough devices" is expedited" 167 and several examples of HIT were removed from the definition of medical device. ${ }^{168}$ President Trump's administration promises to go much further and has taken an avowedly antiregulatory posture ${ }^{169}$ aimed at the "deconstruction of the administrative state." President Trump's FDA Commissioner Scott Gottlieb has already announced a Digital Health Innovation Action Plan that will move digital health products to a post-marketing surveillance model and increasingly rely on third-party certification. ${ }^{171}$ Indeed, certification and other forms of soft law or private ordering are likely to be increasingly important substitutes for formal regulation of emerging healthcare data technologies.

Different models promote various goals. For example, accreditation and curation models fixate on trust, while ratings or recommendation models promote transparency. Some healthcare data technologies are distributed exclusively through app stores owned by platform owners. The question then becomes whether the platform owner has sufficient incentive to police its store for quality and safety. For example, Google, whose own business model rotates around collecting user data, has only very basic rules on the Android store. ${ }^{172}$

166. 21 st Century Cures Act, Pub. L. No. 114-255, 130 Stat. 1033 (2016).

167. $\S 3051,130$ Stat. at 1121 (codified as amended at 21 U.S.C. $360 \mathrm{e}-3$ (2017)).

168. $\S 3060,130$ Stat. at 1130-31 (codified as amended at 21 U.S.C.360j(o)(1) (2017)).

169. See, e.g., Bourree Lam, Trump's 'Two-for-One' Regulation Executive Order, ATLANTIC (Jan. 30, 2017), https://www.theatlantic.com/business/archive/2017/01/trumps-regulation-eo/515007 (cxplaining that the Trump Administration plans to deregulate "massively for small businesses and for large businesses").

170. Philip Rucker \& Robert Costa, Bannon Vows a Daily Fight for 'Deconstruction of the Administrative State, 'WASH. POST (Feb. 23, 2017), https://www.washingtonpost.com/politics/top-whstrategist-vows-a-daily-fight-for-deconstruction-of-the-administrative-state/2017/02/23/03f6b $8 \mathrm{da}-\mathrm{f} 9 \mathrm{ea}-$ 11 e6-bf01-d47f8cf9b643_story.html.

171. Nathan G. Cortez et al., Questions About the FDA's New Framework for Digital Health, HEALTH AFF. BLOG (Aug. 16, 2017), http://healthaffairs.org/blog/2017/08/16/questions-about-the-fdas-newframework-for-digital-health; U.S. FOOD \& DRUG ADMIN., DIGITAL HEALTH INNOVATION ACTION PLAN 5-6 (2017) [hereinafter 2017 FDA ., DigITAL HEALTH INNOVATION ACTION Plan] https:/www.fda.gov/downloads/MedicalDevices/DigitalHealth/UCM568735.pdf.

172. See Google Play Developer Program Policies, ANDROID, https://www.android.com/developercontent-policy.html (last visited Oct. 10, 2017) (indicating that the policy simply says, "[w]e don't allow unauthorized publishing or disclosure of people's private and confidential information, such as credit card numbers, Social Security numbers, driver's and other license numbers, non-public contacts, or any other information that is not publicly accessible"); There are some additional restrictions associated with the Google Ad platform. See id. (stating that policies similarly apply to ads placed on the Google platform).For an example of the apparent reach of Google's own data processing regarding the IoT, see Jonny Lieberman, 13 Cool Facts About the 2017 Porsche 911, MOTORTREND . (Oct. 5, 2015) http://www.motortrend.com/features/mt_hot_list/13-cool-facts-about-the-2017-porsche-911/ (noting that 
However, Apple professes not to be in that business ${ }^{173}$ and, whether because of principle or product differentiation (or both), seems committed to its consumers' privacy and security, ${ }^{174}$ even though that increases its costs with regard to providing personalized and context-aware services. ${ }^{175}$ For U.S. iPhone users, Apple's App Store Review Guidelines may provide more protection for usergenerated healthcare data than any extant formal regulatory model. ${ }^{176}$

\section{B. Value's Regulatory Failures}

U.S. regulatory failure can take many different forms (frequently depending on the critic's ideology). Three failure involving aspects of value are discussed below. First, regulations can linger after their sell-date. Products or services (or whatever the subject of regulation) are likely to improve over time or their users may simply become better informed of risks. However, during that timeframe regulatory models can acquire a form of permanence detached from any original regulatory purpose, tempting stakeholders to leverage them for other, often anticompetitive purposes. Second, regulation often offers too little, too late. As already discussed ${ }^{177}$ there is considerable disagreement when to regulate technologies, for example at the leading or trailing edge. Third, some regulatory models simply lack traction in a system that professes to place its faith in markets. For example, price controls or new technology assessments that threaten a market-based approach are a very hard sell in the U.S.

\section{The Professional Distribution Stack}

Healthcare is not the only industry that suffers because of inefficient distribution models supported by outdated laws. For example, the automobile

Google tried to form an agreement with Porsche where they would receive vehicle speed, throttle position, coolant and oil temp, and engine revs whenever Android Auto was activated).

173. See Apple's Commitment to Your Privacy, APPLE, http://www.apple.com/privacy (last visited Oct. 8, 2017) (noting that Apple uses strong encryption and strict policies to govern the handling of user data).

174. Rich Mogull, Why Apple Really Cares about Your Privacy, MACWORLD (June 25, 2014, 4:00 AM), http://www.macworld.com/article/2366921/why-apple-really-cares-about-your-privacy.html (stating that Apple does not share personal information with third parties for marketing purposes).

175. See, e.g., Julia Love, Exclusive: Apple Ups Hiring, but Faces Obstacles to Making Phones Smarter, REUTERS (Sept. 7, 2015, 9:08 AM), https://www.reuters.com/article/us-applemachinelearning/exclusive-apple-ups-hiring-but-faces-obstacles-to-making-phones-smarter-

idUSKCNOR71H020150907 (stating that "Apple largely relies on analyzing the data on each user's iPhone rather than sending it to the cloud, where it can be studied alongside information from millions of others").

176. See App Store Review Guidelines $\S$ 5.1.3, APPLE DEVELOPER, https://developer.apple.com/appstore/review/guidelines/\#healthkit (last visited Oct. 8, 2017) (providing additional rules for health, fitness, and medical data to ensure customer privacy protection).

177. See supra text accompanying note 158 . 
industry incurs unproductive costs because of franchise laws. ${ }^{178}$ Over the years, however, healthcare has accumulated an embarrassing number of barriers, from Certificate of Need Laws, ${ }^{179}$ to the Corporate Practice of Medicine doctrine, ${ }^{180}$ and state (rather than federal) licensure. ${ }^{181}$

The FDA itself acknowledges it "does not regulate the practice of medicine - how and which physicians can use a device." 182 However, FDA decisionmaking does determine the extent that a device is subject to regulated (including physician) distribution. ${ }^{183}$ Oversimplified, a product may be unregulated (not a "device"), regulated as a device but approved for over-the-counter (OTC) distribution, or regulated as a prescription-only $(\mathrm{Rx})$ device. Few medical devices are available OTC. ${ }^{184}$ In particular the FDA approves very few in vitro diagnostic products (IVDs) for OTC distribution (there are notable exceptions such as pregnancy testing kits). ${ }^{185}$ In a sense, therefore, the FDA preserves professional hegemony: if devices tend to do what doctors do (diagnose, treat, etc.) or are sufficiently high risk that they should only be used by professionals, then FDA regulation will consign them to distribution through professional

178. See Daniel A. Crane, Tesla, Dealer Franchise Laws, and the Politics of Crony Capitalism, 101 IOWA L. REV. 573, 576, 592 (2016) (cxplaining the development of U.S. franchising laws meant to restrict car manufacturers and dealers to protect consumers).

179. CON-Certificate of Need State Laws, NAT'L CONF. OF ST. LeGiSlaTures (Aug. 25, 2016); http://www.ncsl.org/rescarch/health/con-certificate-of-need-state-laws.aspx ("Certificate of Need (C.O.N.) programs are aimed at restraining health care facility costs and facilitating coordinated planning of new services and facility construction.").

180. Corporate Practice of Medicine, AM. HEALTH LAW Ass'N, https://www healthlawyers.org/hlresources/Health\%20Law\%20Wiki/Corporate\%20Practice\%20of\%20 Medicine.aspx (last visited Oct. 10, 2017) (prohibiting "a business corporation from practicing medicine or employing a physician to provide professional medical services").

181. See generally Credentialing, Licensing, and Education, NAT'L CTR. COMPLIMENTARY \& INTEGRATIVE HEALTH, https://nccih.nih.gov/health/decisions/credentialing.htm (last modified Mar. 25, 2016) (noting that state licensing regulations for complementary health practitioners tend to vary "from state to state and from discipline to discipline").

182. Overview of Devices Regulation, U.S. FOOD \& - DRUG ' ADMIN., https://www.fda.gov/MedicalDevices/ProductsandMedicalProcedures/HomeHealthandConsumer/Home UseDevices/ucm204884.htm (last updated Aug. 14, 2015).

* 183. Nicolas Terry, Hearing Aids and The Sound Of Mobile Health Disruption, HeAtTH AfF. BLOG (Jun. 17, 2016), http://healthaffairs.org/blog/2016/06/17/hearing-aids-and-the-sound-of-mobile-bealthdisruption/

184. See, Medical 'Devices and Accessories,' 'AMAZON, https://www.amazon.com/gp/help/customer/display.html?ie=UTF8\&nodeld=200277400 (lasit visited Sept. 5, 2017) (displaying a list of devices where only a few are approved by the FDA to be sold over-thecounter, whereas, most medical devices are only available by prescription).

185. See In Vitro Diagnostics,

FDA, http://www.fda.gov/medicaldevices/productsandmedicalprocedures/invitrodiagnostics/ (last updated Jan. 13,2017 ) (explaining that in vitro diagnostic tests are used at in the laboratory, in health professional settings, and in the home); see also Thomas Jull, FDA Approval Trending for In Vitro Diagnostics Over the Counter Devices, LiNKEDIN fig.2 (Jan. 18, 2017), https://www.linkedin.com/pulse/fda-approvaltrending-vitro-diagnostics-over-counter-devices-jull (noting that compiled data from the FDA's approval of IVD OTC products indicates a trend that approvals are on the rise). 
channels.

History is replete with regulators spurning disruptive technologies because of concerns about their impact on incumbents. For example, in the late $1600 \mathrm{~s} \mathrm{a}$ curate named William Lee, apparently spurned by a lady who preferred knitting to dating, spent three years inventing a stocking-weaving machine. ${ }^{86}$ It transpired that Queen Elizabeth I was a big fan of knitted stockings so Lee travelled to London to request a patent from the queen. According to lore he was turned down because the queen was concerned that individual stocking makers would be made redundant. ${ }^{187}$

The stakes tend to be higher when the work of professional guilds is involved. Five hundred years after the Queen disappointed curate Lee the American Society of Anesthesiologists campaigned against ${ }^{188}$ the Sedasys anesthesiology machine discussed above. ${ }^{189}$ After a couple of denials the FDA approved robot for colonoscopy and esophagogastroduodenoscopy procedures. ${ }^{190}$ The anesthesiologists begrudgingly published guidelines consistent with the device's limited scope of practice. ${ }^{191}$ However, sales were modest with the device being used in just four hospitals even though it provided

\footnotetext{
186. Spurned love leads to Knitting Invention, BBC (July 22, 2009), $\mathrm{http}: / /$ www.bbc.co.uk/nottingham/content/articles/2009/07/20/william_lee_knitting_frame_feature.shtml
}

187. Id.

188. See Todd C. Frankel, It's Game over for the Robot Intended to Replace Anesthesiologists, WASH. PosT (Mar. 28, 2016), https://www.washingtonpost.com/news/the-switch/wp/2016/03/28/its-game-overfor-the-robot-intended-to-replace-anesthesiologists/?utm_term $=435 \mathrm{ae} 7 \mathrm{f} 4717 \mathrm{~d}$ (explaining that the Amcrican Socicty of Anesthesiologists campaigned against the Sedasys anesthesiology machine before it entered the market).

189. See supra text accompanying note 97; see also Anthony Cuthbertson, Plug Pulled on Robot Doctor after Humans Complain, NEwsweEK (Mar. 30, 2016, 6:53 AM), http:/www.newsweek.com/plugpulled-robot-doctor-after-humans-complain-442036 (explaining that the Sedasys is a "computerized sedation system" that provides "anesthesiology services to endoscopy and colonoscopy patients.").

190. See Letter from Christy Foreman, Office Dir., Office of Device Evaluation, Ctr. for Devices \& Radiological Health, to Neelu Medhekar, Dir. Regulatory Affairs U.S. FOOD \& DRUG ADMIN., SEDASYS Computer-Assisted Personalized Sedation System, Approval Order, (May 3, 2013), https://www.accessdata.fda.gov/cdrh_docs/pdf8/p080009a.pdf (indicating that the FDA approved Sedasys in 2013); see also Rachel Fobar, Meet the Machine That Could Replace Anesthesiologists, POPULAR SCI. (May 12, 2015), http://www.popsci.com/meet-machine-could-replace-anesthesiologists (indicating that the FDA approved Sedasys in 2013 after first rejecting it in 2010).

191. See American Society of Anesthesiologists (ASA) Offers Recommendations for Using SEDASYS, AM. SOC'Y OF ANESTHESIOLOGISTS (Jan. 22, 2014), http://www.asahq.org/about-asa/newsroom/newsreleases/2014/01/sedasys (explaining that the ASA has issued a guidance document with recommendations for "computer-assisted personalized sedation" systems); see Anesthesia Automation May Widen GI Professionals' Use of Propofol, P\&T COMMUNITY (Sept. 30, 2015), https://www.ptcommunity.com/news/2015-09-30-000000/anesthesia-automation-may-widen-giprofessionals-use-propofol ("The American Society of Anesthesiologists issued guidelines for the use of the device in 2014 , but did not endorse the system."). 
a dramatic cost-savings compared to a human anesthesiologist, and in March 2016, the manufacturer pulled the product from the market. ${ }^{192}$

The American Medical Association frequently has opposed disaggregated healthcare models such as retail clinics citing increased costs through duplicative services and quality concerns. ${ }^{193}$ Equally, organized medicine has not been friendly towards technologically-mediated care such as traditional telemedicine ${ }^{194}$ or contemporary video "visits." 195 The courts seem to support our antitrust agencies when they occasionally poke the guilds. For example, Justice Kennedy refused to apply state antitrust immunity when government "abandon[s] markets to the unsupervised control of active market participants, whether trade associations or hybrid agencies." ${ }^{\prime 196}$ And such scrutiny does seem to produce results such as the recent liberalizing of the Texas telemedicine statute. ${ }^{197}$.

These examples are symptoms of a deeper issue rooted in the history of physician hegemony, ${ }^{198}$ a professional distribution "stack" that insinuates medical professionals into the distribution of products. Here the guilds leverage outdated regulatory models to protect income streams in cases where, arguably, the technologies have become safer or new alternative technologies are available. Historic examples include the distribution of contact lenses, ${ }^{199}$, products that are

192. Cuthbertson, supra note 189; Esther Inglis-Arkell, Robot Anesthesiologists Lose Their Jobs Because of Humans, GIZMODO (Mar. 29, 2016, 7:05 PM), http://gizmodo.com/robot-anesthesiologistslose-their-jobs-because-of-huma-1767844468.

193. But see Rachel O. Reid \& Ateev Mehrotra, Commentary, Primary Care Practice Response to Retail Clinics, 15 AMA J. ETHICS 937,938 (2013) ("Retail clinics have been shown to deliver care that is comparable in quality and lower in cost than primary careOffices."); see also Bruce Japsen, AMA Takes on Retail Clinics, CHI. TRIB. (June 25, 2007), http://articles.chicagotribune.com/2007-0625/business/0706240383_1_retail-clinics-ama-doctors-groups (explaining that the AMA believes that retail clinics endanger patient care).

194. See, e.g., Abby Goodnough, Texas Medical Panel Votes to Limit Telemedicine Practices in State, N.Y. Times (Apr. 10, 2015), https://nyti.ms/1Nn03B3 (explaining that the Texas Medical Board had taken steps to impose restrictions on the use of telemedicine due to patient safety concerns).

195. See, e.g., Jayne O'Donnell, Video Visits Blocked Despite Doctor Shortage, USA ToDAY (Dec. 7, 2014, 3:31 PM), https://www.usatoday.com/story/news/nation/2014/12/07/telemedicine-state-laws-ruralhospitals-specialists/70094828/ (explaining that although there is no organized opposition to video medicine, nearly thirty states have laws which discourage doctors from practicing video medicine).

196. N.C. State Bd. of Dental Exam'rs v. F.T.C., 135 S. Ct. 1101, 1117 (2015)

197. Eric Wicklund, Texas Telemedicine Law Prompts Feds to Close Medical Board Probe, MHEALTH INTELLIGENCE (June 29, 2017),https://mhealthintelligence.com/news/texas-telemedicine-law-prómpts-

$\therefore$ : feds-to-close-medical-board-probe (explaining that the FTC's probe into Texas' prohibitive telemedicine regulations ended after Texás' governor signed less restrictive telemedicine regulations).

198. See, e.g., Paul StarR, The Social Transformation of AMERICAN Medicine 21-24 (1982) (explaining that the prohibition of alternative practitioners and the limited supply of physicians has led to a monopoly of the medical profession by physicians).

199. See FTC Contact Lens Rule, 16 C.F.R. $§ 315.1-3$ (2015) (requiring ophthalmologists and optometrists to provide their patients with contact lens prescriptions, ending the tying of an eye examination to the provision of lenses); see Christopher Versace, The FTC Finally Sees the Light on Contact Lenses, ForBES (Jan. $17, \quad 2017, \quad 3: 29 \quad$ PM), 
sold inexpensively OTC outside the U.S., ${ }^{200}$ and there are growing controversies regarding the exclusive distribution of hearing aids by physicians or audiologists ${ }^{201}$ and online vision tests. ${ }^{202}$

\section{Under Regulated Data Protection}

The Breach of Confidence ${ }^{203}$ and Intrusion into Seclusion ${ }^{204}$ torts may have sufficed in a world of paper files locked up in the file cabinet of Marcus Welby, $M . D .{ }^{205}$ However, HIPAA privacy and security rules ${ }^{206}$ were needed to deal with the new world of diagnosis or treatment code sets and paperless billing. ${ }^{207}$ HIPAA was revised by HITECH $^{208}$ to reflect the consensus policy decision to expand HIT, a decision itself accelerated by the HITECH meaningful use

https:/www.forbes.com/sites/chrisversace/2017/01/17/the-ftc-finally-sees-the-light-on-contactlenses/\#8a735746dde0 (explaining that contact lens manufacturers have a monopoly over the market because optometrists and ophthalmologists force consumers to buy contact lenses directly from them).

200. See, e.g., Elisabeth Rosenthal, When a Health Journalist Walks into a Pharmacy..., MEDIUM (July 24, 2017) https://medium.com/@RosenthalHealth/when-a-health-journalist-walks-into-a-pharmacy$6 \mathrm{cb} 60 \mathrm{~b} 519 \mathrm{~b} 5 \mathrm{c}$ (explaining that a prescription for hydrocortisone suppositories, without insurance, costs $\$ 277$ in the United States, while in the U.K., the same medicine is available over-the-counter for approximately $\$ 7.50$ ).

201. See generally Terry, supra note 201 (explaining that the FDA's regulation requiring hearing aids to be distributed solely by physicians and audiologists has led to low innovation and high costs); see generally Over-the-Counter Hearing Aid Act of 2017, S. 670, 115 th Cong. $\$ 2$ (a)-(c) (creating a category of over-the-counter hearing aids and regulations for hearing aids under that category).

202. See, e.g., Bradley George, Online Eye Exam Site Makes Waves in Eye Care Industry, NPR (May 25, 2016, 4:54 AM), http://www.npr.org/sections/alltechconsidered/2016/05/25/479346651/online-eyeexam-site-makes-waves-in-eye-care-industry (explaining that many states and the American Optometric Association are against online vision tests because online vision tests cannot replace the benefits of a traditional eye exams); see also Meg Graham, Opternative Fighting States That Try to Ban Its Online Eye Exams, CHI. TRIB. (Apr. 5, 2016, 2:50 PM), http://www.chicagotribune.com/bluesky/originals/ctopternative-legislation-online-exams-bsi-20160405-story.html (explaining that the FDA has not approved online vision tests and the American Optometric Association opposes online vision tests).

203. See RESTATEMENT (SECOND) OF TORTS $\S \S 652$ A-652I (AM. L. INST. 1977) (setting out the law regarding invasion of privacy); see RESTATEMENT (THIRD) OF RESTITUTION AND UNJUST ENRICHMENT $\S$ 43 (AM. L. INST. 2011) (establishing that a Breach of Confidence tort arises when a person obtains a benefit through "breach of [a] ... duty imposed by a relation of trust and confidence").

204. RESTATEMENT (SECOND) OF TORTS $\$ 652 \mathrm{~B}$ (1966).

205. Marcus Welby, M.D., TV GUIDE, http:/www.tvguide.com/tvshows/marcus-welbymd/cast/202918/ (last visited Sept. 7, 2017) ("Popular Emmy-winning drama about a sage general practitioner and his headstrong younger partner.").

206. See generally 45 C.F.R. $\S \S 160,162,164$ (2016) (establishing privacy and sccurity regulations to protect electronic health information).

207. HIPAA for Professionals, U.S. DEP'T OF HEALTH \& HUM. SERVS., https:/www.hhs.gov/hipaa/for-professionals/index.html (content last reviewed June 16, 2017) (explaining that HIPAA required the Department of Health and Human Services to adopt national standards for electronic health care transactions).

208. See American Recovery and Reinvestment Act of 2009, Pub. L. No. 111-5, $\S 13001,123$ Stat. 115,226 (enacting the Health Information Technology for Economic and Clinical Health (HITECH) Act). 
subsidies for the purchase of EHRs. ${ }^{209}$ HIPAA and HITECH were written to protect patient data collected by healthcare providers, HIPAA “covered entities," and their "business associates."210

Even within the HIPAA-protected space, security and data quality issues are close to a breaking point as the unprepared healthcare industry contemplates data losses that cause patient quality/safety adverse events ${ }^{211}$ and attacks on data clusters that result in cascading failures. ${ }^{212}$ However, increasingly clinical, wellness, fitness, and medically-inflected data are collected and processed outside of that HIPAA-protected space by entities to whom HIPAA does not apply. ${ }^{213}$. Indeed, that would be true of most of the healthcare data technologies discussed herein. ${ }^{214}$ Certainly, this is the case with most health apps that are built by technology companies and sold through app stores. ${ }^{215}$ It is also true of wearables and the IoT: ${ }^{216}$ As ONC recognized in a-2016 report to "Congress; "Wearable fitness trackers, health social media, and mobile health apps are premised on the idea of consumer engagement. However, our laws and regulations have not kept pace with these new technologies." 217 These apps and devices are operating in the HIPAA-free zone with little or no regulation as to

209. Meaningful Use Definitions \& Objectives, HEALTHIT.gOV, https://www.healthit.gov/providersprofessionals/meaningful-use-definition-objectives (last visited Sept. 7, 2017) (explaining that "meaningful use is using certified electronic health record (EHR) technology to," for example, "improve quality," "engage patients," "maintain privacy and security," and improve public health); see Ashish Jha, The Final State of Meaningful Use Rules: Will EHRs Finally Pay Off?, HEALTH AfF. BLOG (Mar. 25, 2015), http://healthaffairs.org/blog/2015/03/25/the-final-stage-of-meaningful-use-rules-will-chrs-finallypay-off/ (explaining that HITECH's meaningful use incentives for adopting EHRs were prompted by the desire to improve the "safety, effectiveness, and efficiency of health care delivery").

210. 45 C.F.R. $\S 160.103$ (2016); Summary of the HIPAA Security Rule, U.S. DEP'T OF HEALTH \& HUM. SERVS., https://www.hhs.gov/hipaa/for-professionals/security/laws-regulations/index.html (content last reviewed July 26, 2013).

211. See, e.g., Jose Pagliery, 90\% of Hospitals and Clinics Lose Their Patients' Data, CNN (Aug. 20, 2014, 11:06 AM), http://money.cnn.com/2014/08/20/technology/security/hospitals-data/index.html (explaining that between 2012 and 2013, 90\% of healthcare providers exposed or lost their patients' data because they were not adequately protecting medical files and because they were using outdated technology that is vulnerable to attack).

212. See, e.g., Casey Newton, How a Typo Took down S3, the Backbone of the Internet, VERGE (Mar. 2, 2017, 1:24 PM), http://www.theverge.com/2017/3/2/14792442/amazon-s3-outage-cause-typo-internetserver? (cxplaining how an Amazon server outage caused a ripple effect that led to the failures of several other Amazon services, such as Quora, Trello, and IFTTT).

213. See Terry, supra note 1 , at 181-82 (explaining that many mobile health apps operate outside of HIPAA-regulàted space).

- 214. See id. at 182-83 (explaining wearable fitness trackers, health social media, and mobile health apps are not subject to regulations).

215. Id. at 181 .

216. See Terry, supra note 61 , at $342-45$ (cxplaining that HIPAA protections do not extend to wearables and the loT).

217. U.S. DeP'T OF HeAlTh \& HuM. ServS., EXamining OVERSight OF THE PRIVACY \& SECURITY of Health DAta Collected by ENTIties Not Regulated by HIPAA 32 (2016), https://www.healthit.gov/sites/default/files/non-covered_entities_report_june_17_2016.pdf. 
how they should share data with third parties or what the security is expected of any off-device data storage.

As they proliferate robots undoubtedly will pose similar privacy and security risks. Like their fellow members of the Internet of Things they tend to be "always on" and equipped with copious sensors. Robots, like other IoT devices may evolve or acquire new sets of privacy threats, unsettling privacy expectations. For example, a cordless vacuum scurrying around your home likely would soon fade into the background, dismissed from the consciousness of privacy strategies. What, however, if several years after the robot "disappeared" it was re-tasked with collecting data about your home, with the robot developer selling these data about your physical environment? ? $^{218}$

Robots also pose similar regulatory indeterminacy problems. Thus, inside healthcare institutions laundry and pharmacy tugs and pickers designed to separate waste products with toxic properties that incidentally capture patient data likely will face HIPAA regulation. The same is probably true of hospitalbased telepresence or carer robots. However, as we give robots access to our homes and places of employment we will not only lose HIPAA protection but also increase data protection and related threats. As Ryan Calo notes, "Robots' social meaning could have a profound effect on privacy and the values it protects..."219 As we interact more with our robots so we tend to anthropomorphize them and act somewhat differently, impacting our privacy strategies. This tendency could become more likely with robots into which human characteristics have been designed.

As an example, consider the therapeutically positive experience of patients at a Boston hospital. Some children recovering from urological surgery need less care than hospitalization but more than parents at home can provide, and so the hospital sometimes sends a VGo remote telepresence robot ${ }^{220}$ home with the child. Children apparently connect positively with the robot to the benefit of increased engagement in their health. ${ }^{221}$ HIPAA privacy and security presumably would apply to hospital-provided robots although it is less clear that HIPAA would apply to non-child-health-related data (say some parental financial

218. See, e.g., Jan Wolfe, Roomba Vacuum Maker iRobot Betting Big on the 'Smart' Home, REUTERS (July 24, 2017, 7:09 AM), http:/www.reuters.com/article/us-irobot-strategy-idUSKBN1A91A5 (explaining the increasing popularity and compatibility of smart home interfaces as well as the potential of simultaneous localization and mapping technology, as used in robovacs).

219. M. Ryan Calo, Robots and Privacy, in RoBOT ETHICS: THE ETHICAL AND SOCIAL IMPLICATIONS OF ROBOTICS 187, 195 (Patrick Lin et al. eds., 2012).

220. See Patient Monitoring, VGo, http://www.vgocom.com/patient-monitoring (last visited Sept. 13, 2017) (describing the benefit of VGo technology which allows for two-way audio video communication between patients and providers).

221. Erin McCann, Health IT Promises New Paradigm of Patient Care, HEALTHCARE IT News (Sept. 12, 2012, 11:49 AM), http://www.healthcareitnews.com/news/health-it-promises-new-paradigm-patientcare. 
transaction or spousal abuse) observed by the robot. And, obviously, if a family purchased their "synth" from a big box store, ${ }^{222}$ HIPAA protections would not apply, leaving the family hoping for help from some state privacy law would or that the over-extended FTC would investigate their violated privacy. ${ }^{223}$

The data-crunching, AI-based robots, and machine-learning systems of our near future will raise the privacy stakes still higher. Big data brokers have been voracious consumers of healthcare data, including de-identified clinical data and identifiable medically-inflected data. ${ }^{224}$ Only a few of their activities are subject to specific regulation, for example under The Fair Credit Reporting Act (FCRA) ${ }^{225}$ Thereunder the FTC has been able to take action against data custodians that, for example, sell consumer information to those who have no legitimate need for it. ${ }^{226}$ Several branches of government have decried the data industry's adverse impact on privacy, yet there has been no meaningful progress towards legislation to better regulate the industry. ${ }^{227}$ As I have noted elsewhere, unregulated big data goes far beyond the enabling of targeted advertising. Health scoring, body scores, and other data segmentation carry very real threats of discrimination. ${ }^{228}$ Data analytics have immense potential for improving healthcare and it is no surprise that healthcare entities are entering into datasharing arrangements with data-mining companies. However, as the controversy over the relationship between Alphabet's DeepMind and the Royal Free NHS Trust hospitals ${ }^{229}$ and the subsequent scathing report by the UK Information

222. Humans, IMDB, http://www.imdb.com/title/tt4122068/ http:/www.vgocom.com/patientmonitoring (last visited Dec. 17, 2017) (television series about a futuristic parallel universe where the "must-have gadget" is a "Synth'- a highly-developed robotic servant that's so similar to a real human it's transforming the way we live").

223. See 15 U.S.C. $\$ 45(a)(1)$-(2) (2016) ("[U]nfair methods of competition in or affecting commerce, and unfair or deceptive acts or practices in or affecting commerce, are hereby declared unlawful:").

224. See generally Nicolas P. Terry, Big Data Proxies and Health Privacy Exceptionalism, 24 HEALTH MATRIX 65, 80, 102 (2014) (examining that "big data" brokers are consuming large amounts of health data and because big data facilitates the creation of health data proxies that are relatively unprotected, the level of protection for health data is ultimately reduced).

225. See 15 U.S.C. $\S 1681(\mathrm{~g})(1)-(4)$ (2016) (regulating privacy, accuracy, and fairness in the collection of individual credit information by credit reporting agencies).

226. See, e.g., Data Broker Defendants Settle FTC Charges They Sold Sensitive Personal Information to Scammers, FED. TRADE COMM'N (Feb. 18, 2016), https://www.ftc.gov/news-events/pressreleases/2016/02/data-broker-defendants-settle-ftc-charges-they-sold-sensitive (announcing a group of defendants settled with the FTC admitting they knowingly provided scammers with hundreds of thousands of consumers' sensitive personal information).

227. See generally Nicolas Terry, Navigating the Incoherence of Big Data Reform Proposals, 43 J.L. MED. \& ETHICS 44 (2015) (explaining that although the federal government has issued several reports which favor an increase in big data regulation, there has been limited efforts in actually issuing regulations).

228. Terry, supra note 1 , at 180-81, 199 (explaining concerns of mobile health apps that share data and are not subject to HIPAA regulations).

229. See Ben Heather, Google DeepMind and Royal Free in Five-Year Deal, Digital hEALTH (Nov. 22, 2016), https://www.digitalhealth.net/2016/11/google-deepmind-and-royal-free-in-five-year-deal/ (describing the deal between Google-owned artificial intelligence company, DeepMind, and the Royal 
Commissioner ${ }^{230}$ make clear, providers must do far more to preserve the autonomy and privacy of their patients.

\section{Cost-Effectiveness Determination}

Assuming that an increasing number of healthcare data technologies will present and that we can overcome product safety and data quality issues, other Value issues will arise. One of the most important is the determination whether any such technology is cost-effective. Both effectiveness and efficiency are IOM Quality Domains. "Effectiveness" is defined as relating to "providing care processes and achieving outcomes as supported by scientific evidence," and "Efficiency" as "maximizing the quality of a comparable unit of health care delivered or unit of health benefit achieved for a given unit of health care resources used."231

There is a prevailing but somewhat naïve hypothesis that, referencing the original triangle, health services disaggregation or technologies reduce cost while increasing access. The evidence tends to disagree. ${ }^{232}$ Indeed, U.S.

Free NHS Foundation Trust to further develop the acute kidney injury detecting app, "Streams"); see also Hal Hodson, Revealed: Google AI has Access to Huge Haul of NHS Patient Data, NEw SCIENTIST (Apr. 29, 2016), https://www.newscientist.com/article/2086454-rcvealed-google-ai-has-access-to-huge-haulof-nhs-patient-data/ (explaining how the partnership between Google's DeepMind and the NHS extends beyond what was publically announced, giving Google access to millions of patients health information over several years);see also Natasha Lomas, Concerns Raised Over Broad Scope of DeepMind-NHS Health Data-Sharing Deal, TECHCRUNCH (May 4, 2016), https://techcrunch.com/2016/05/04/concernsraised-over-broad-scope-of-deepmind-nhs-health-data-sharing-deal/ (explaining concerns about the vast amount of data-sharing occurring between DeepMind and the NHS, after it was revealed they have access to extensive patient data, rather than just acute kidney injury data); see also Natasha Lomas, DeepMind Wants its Healthcare AI to Charge by Results-But First it Needs Your Data, TECHCRUNCH (Sept. 20, 2016), https://techcrunch.com/2016/09/20/deepmind-wants-its-healthcare-ai-to-charge-by-results-butfirst-it-needs-your-data/ (explaining DeepMind's announcement of a future Patient Portal that would allow for increased data collection and aims to boost engagement and improve health outcomes).

230. See Royal Free - Google DeepMind Trial Failed to Comply with Data Protection Law, INFo. COMM'RS OFF. (July 3, 2017), https://ico.org.uk/about-the-ico/news-and-events/news-andblogs/2017/07/royal-free-google-deepmind-trial-failed-to-comply-with-data-protection-law/ ("There's no doubt the huge potential that creative use of data could have on patient care and clinical improvements, but the price of innovation does not need to be the erosion of fundamental privacy rights.").

231. Understanding Quality Measurement, AgENCY OF HEALTHCARE RESEARCH \& QUALITY (2012), https://www.ahrq.gov/professionals/quality-paticnt-safety/quality-

resources/tools/chtoolbx/understand/index.html.

232. See, e.g., J. Scott Ashwood et al., Direct-To-Consumer Telehealth May Increase Access to Care But Does Not Decrease Spending, 36 HEALTH AFF. BLOG 485, 485-91 (2017) (examining the patterns of utilization of direct-to-consumer telchealth and noting that while telehealth may offer increased paticnt access, it also increases overall health care spending because it is more likely to result in follow-up appointments and increases the total number of patient visits); see also M. Thygeson et al., Use and Costs of Care in Retail Clinics Versus Traditional Care Sites, 27 HEALTH AFF. BLOG 1283, 1287-91 (2008) (observing utilization trends, patient mix, and cost of care at retail clinic chains as compared to other care settings and noting that its impact on overall quality and cost remains undetermined); see also RAND CORP., THE EVOLVING ROLE OF RETAIL CLINICS 3 (2016), https://www.rand_org/content/dam/rand/pubs/research_briefs/RB9400/RB9491- 
regulatory systems are particularly poor at analyzing cost effectiveness or even addressing comparative effectiveness questions. For example, although the FDA does address effectiveness in the sense of efficacy, its pre-marketing scrutiny does not compare that effectiveness with drug, device, or clinical benchmarks. Neither does the FDA assess the dollar value of the proposed new drug or device. technology.

Outside of the professional distribution stack, the FTC's scrutiny is limited to scientific efficacy. In POM Wonderful the DC Circuit upheld the agency's position that randomized and controlled human clinical trials were required to adequately substantiate claims that consumption of pomegranate-based products could treat, prevent, or reduce the risk of heart disease, prostate cancer, and erectile dysfunction. ${ }^{233}$ This is the standard that has been applied by the agency in the app cases involving melanoma detection ${ }^{234}$ and eyesight improvement. ${ }^{235}$ :

In contrast, New Technology Assessment (NTA) is' a crucial part of healthcare policymaking in other industrialized countries. For example, The National Institute for Health and Care Excellence (NICE) in the UK ${ }^{236}$ and Institute for Quality and Efficiency in Health Care (IQWiG) in Germany evaluate new drugs on the basis of their comparative or cost effectiveness. Their findings determine whether such drugs are included in the national formulary (UK) or subject to reference pricing (Germany). However, there are few regulatory analogs in the U.S. and the work of the closest one, the Patient-Centered

2/RAND_RB9491-2.pdf (explaining how despite being promoted as cost-saving vehicles, retail clinics actually increase health care utilization and spending); see also Carolyn Y. Johnson, Free-Standing ERs Offer Care without the Wait. But Patients Can Still Pay $\$ 6,800$ to Treat a Cut, WASH. POST. (May 7, 2017), https://www.washingtonpost.com/business/economy/free-standing-cmergency-rooms-offercostly-convenience/2017/05/07/6255d052-2b98-11e7-b605-33413c691853 story.html? (disputing the conception that free-standing emergency rooms offer less expensive services than hospital emergency rooms).

233. POM Wonderful, LLC v. FTC, 777 F.3d 478, 484-85 (D.C. Cir: 2015), cert denied, 136 S. Ct. 1839 (2016).

234. See FTC Cracks Down on Marketers of "Melanoma Detection" Apps, FED. TRADE COMM'N (Feb. 23, 2015), https:/www.ftc.gov/news-events/press-releases/2015/02/ftc-cracks-downmarketers-melanoma-detection-apps (explaining how the FTC's Bureau of Consumer Protection requires app developers and marketers to have scientific evidence to support any health or disease claims asserted for their apps).

235. FTC Charges Marketers of 'Vision Improvement' App with Deceptive Claims, FED. TRADE COMM'N (Sept. 17, 2015), https://www.ftc.gov/news-events/press-releases/2015/09/ftc-chargesmarketers-vision-improvement-app-deceptive-claims (explaining how the FTC ordered marketers and developers of the app "Ultimeyes" to substantiate its claims about the app's ability to improve users' vision with legitimate scientific evidence).

236. What We Do, NAT'L INST. FOR HEALTH \&. CARE EXCELLENCE, https://www.nice.org.uk/about/what-we-do (citing the use of evidence-based guidance in advising heath-care workers) (last visited Oct. 10, 2017); Responsibilities and Objectives of IQWiG, INST. OF QuAliTY \& EFFICIENCY IN HEALTH CARE, https://www.iqwig.de/en/about-us/responsibilities-andobjectives-of-iqwig.2946.html (citing the use of evidence-based reports for evaluating drugs) (last visited Oct. 10, 2017). 
Outcomes Research Institute (PCORI), is fatally hampered by the ACA's prohibition on the use of quality-adjusted life-years (QALYs) as a metric.

Of course, even without formal mechanisms cost-effectiveness frequently will be behind individual, market-based decisions regarding Automation. For example, Alphabet's Verily Life Sciences has retooled around more modest goals and dropped its "moonshot" rehetoric. ${ }^{237}$ Meanwhile, the celebrated partnership between the MD Anderson Cancer Center and IBM to use the Watson platform for cancer research was put on hold after Watson reportedly after cost issues and a failure to meet its goals. ${ }^{238}$ Subsequently, IBM was the subject of a particularly scathing report from an analyst suggesting that Watson was falling behind other technology companies in the deep learning space. ${ }^{239}$

\section{EMPATHY}

Empathy is an intensely human trait. It refers to the ability to understand, feel, or share the feelings of another, ${ }^{240}$ even though some argue it propagates out biases. ${ }^{241}$ In the context of the new triangle it seeks to capture the interpersonal or caring aspects of healthcare most valued by patients (and the majority of doctors ${ }^{242}$ ). It embodies quintessentially human values such as compassion, responsiveness, patient-centeredness, equity and trust. These are traits and values that are important to patients. They are also instrumental, with great value to healthcare providers because, as acknowledged by Tom Beauchamp and James Childress, "Good healthcare often involves insight into the needs of patients and considerate attentiveness to their circumstances, which may derive more from emotional or sympathetic responsiveness than from reason." 243

237. Caroline Chen \& Mark Bergen, Google's Health Moonshot Comes Back to Earth, BLoOMBERG (Apr. 19, 2017, 8:00 AM), https://www.bloomberg.com/news/articles/2017-04-19/google-s-healthmoonshot-comes-back-to-earth?.

238. Matthew Herper, MD Anderson Benches IBM Watson in Setback for Artificial Intelligence in

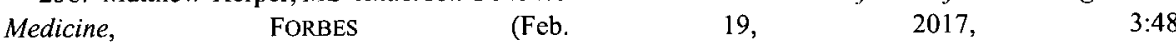
PM), https://www forbes.com/sites/matthewherper/2017/02/19/md-anderson-benches-ibm-watson-insetback-for-artificial-intelligence-in-medicine/\#50720f0f3774.

239. John Mannes, Jefferies Gives IBM Watson a Wall Street Reality Check, TECHCRUNCH (July 13, 2017), https://techcrunch.com/2017/07/13/jefferies-gives-ibm-watson-a-wall-street-reality-check/.

240. See Empathy, MERRIAM-WEBSTER DICTIONARY, https:/www.merriamwebster.com/dictionary/empathy (last visited Dec. 24, 2017)

241. See Paul Bloom, Against Empathy: The Case for Rational Compassion 16 (1st ed. 2016) ("Empathy is the act of coming to experience the world as you think someone else does.").

242. See generally Sandra G. Boodman, How to Teach Doctors Empathy, ATLANTIC (Mar. 15, 2015), https://www.theatlantic.com/health/archive/2015/03/how-to-teach-doctors-cmpathy/387784/ (describing how doctors' empathy is increasingly considered a vital element for the establishment of a strong and trusting doctor-patient relationship).

243. Tom L. Beauchamp \& James F. Childress, PrinCiPles of Biomedical 36 (7th ed. 2013). 
Empathy is not only in tension with Automation but also with Value. This latter, subtler, tension is between our technocratic (often econometric) concept of value and our sociological (philosophical or ethical) sense of values. In contrast, the tension between Automation and Empathy is far more obvious. The more sophisticated deep learning becomes the harder it will be for to understand its reasoning. For example, Deep Patient, a research project at Mount Sinai Hospital exposed 700,000 patient records to AI and then used the deep learning machine the records of new patients. The machine results were consistently strong. Notably, it was able to predict the onset of puzzling psychiatric disorders. However, the research team was unable to determine how that was occurring. The question arises, "at some stage we may have to simply trust AI's judgment or do without using it." ${ }^{244}$ As a result, it is relatively easy to paint an empathyoppositional image of healthcare data technology; say, a virtual hospital populated by patient avatars surrounded by adbots. In passing, recall the dystopian, machine world of The Matrix ${ }^{245}$ and appreciate the irony of GE using the AI-driven villain Agent Smith as the ,hero in its healthcare "brilliant machines" advertising campaign. ${ }^{246}$

One does not have to accept a fully dystopian image of future healthcare ruled by data bots in order to appreciate the tensions between automation and empathy (and value?). Consider a far more benign example, the EHR Meaningful Use program. Policymakers, for probably good reasons, decided that the universal adoption of interoperable EHRs would improve both individual care and, because of their generation of outcomes and other data, population health. Initial application of the original iron triangle would have suggested an increase in healthcare quality (and safety) offset by an initial but declining cost hit because of the government subsidies and some provider costs. ${ }^{247}$ However, as the program unfolded a very different set of problems arose. Essentially, automation created an interpersonal wedge between physicians and patients. The patient became secondary to the enterprise as the EHR and its computer interface dominated the examination room. Indeed, Abraham Verghese coined the word iPatient noting how it "threatened to become the real focus of our attention, while the real patient in the bed often feels neglected, a mere placeholder for the virtual

244. Will Knight, The Dark Secret at the Heart of AI, MIT TECH. REV. (Apr. 11, 2017), https://www.technologyreview.com/s/604087/the-dark-secret-at-the-heart-of-ai/.

245. THE MATRIX (Warner Brothers 1999).

246. See David Gianatasio, The Matrix's Agent Smith Takes a Shine to GE's Brilliant Machines, ADWEEK (Apr. 15, 2013), http:/www.adweek.com/creativity/agent-smith-matrix-takes-shine-gesbrilliant-machines-148584/ (criticizing GE's new advertising campaign featuring The Matrix's Agent Smith for its "brilliant machines" in the health care sector).

247. See Terry, supra note 154, at 9-12 (discussing the history and projected benefits of adopting electronic medical records). 
record." 248 And what is the "solution" of healthcare stakeholders? To assign scribes to doctors who can fill in the EHR fields while the doctor examines the patient, ${ }^{249}$ because nothing says empathy better than showing your new rash to a recent graduate from stenography school! $!^{250}$

The following are some of the issues that impact Empathy in the new triangle and some examples of the tensions and questions that will arise.

\section{A. Will Automation Make Us Rethink Our Conception of Carers and Caring?}

As Kevin Kelly has remarked, "We aren't giving "good jobs" to robots. Most of the time we are giving them jobs we could never do. Without them, these jobs would remain undone." 251 Increasingly, those jobs are going to be in healthcare. Those jobs will not just be custodial (e.g., robot tugs hauling infected laundry) but positions taken over from human carers by comfort robots. ${ }^{252} \mathrm{~A}$ useful starting point, therefore, is to reflect briefly on the current carer industry. Social historian Guian McKee has explained how, in the wake of deindustrialization, the rise of healthcare (and its high costs) increasingly has supported "the economies of communities throughout the country, employing millions of workers." 253 This macroeconomic impact of the healthcare economy has played out particularly strongly in the carer industry with the U.S. employing at least 3.6 million workers with strong growth predicted over the next decade. ${ }^{254}$ Because of high demand in an ageing country and the low pay that carers receive these are jobs that some believe are immune from the disruption by e-commerce

248. Abraham Verghese, Treat the Patient, Not the CT Scan, N.Y. TIMES (Feb. 26, 2011), http:/www.nytimes.com/2011/02/27/opinion/27verghese.html?_r=2\&ref=homepage\&src=me\&pagewan ted $=$ all.

249. See Katic Hafnerjan, A Busy Doctor's Right Hand, Ever Ready to Type, N.Y. TIMES (Jan. 12, 2014), https://www.nytimes.com/2014/01/14/health/a-busy-doctors-right-hand-ever-ready-to-type.html (demonstrating how scribes have allowed physicians a reprieve from note taking).

250. Obviously the meaningful use generation of EHRs had problems other than those impacting empathy. See, e.g., Press Release, Dep't of Justice, Electronic Health Records Vendor to Pay \$155 Million to Settle False Claims Act Allegations (May 31, 2017), https://www.justice.gov/opa/pr/electronic-healthrecords-vendor-pay-155-million-settle-false-claims-act-allegations (explaining how abuse of EHR can cause the submission of falsc claims).. See generally Terry, note 39 at 129 (explaining how HIT suffers from market failures and underperforming products that can result in an array of problems).

251. Kelly, supra note 25.

252. See Sota Home Robot to Care for Japan's Elderly, RoBotics TRENDS (July 31, 2015), http://www.roboticstrends.com/article/sota_home_robot_to_care_for_japans_elderly/Houschold? (explaining how Japan's largest telecommunications company has created a robot that interacts with other smart objects in the home by turning on air conditioners and urging people to drink water).

253. Guian McKee, What's the Connection Between the Baltimore Riots and the Affordable Care Act?, WASH. POST (Dec. 3, 2015), https:/www.washingtonpost.com/news/monkey-cage/wp/2015/12/03/thisexplains-how-the-baltimore-riots-and-the-affordable-care-act-are-two-sides-of-one-problem/?

254. Soo Oh, The Future of Work is the Low-Wage Health Care Job, Vox (July 3, 2017, 10:00 AM), https://www.vox.com/2017/7/3/15872260/health-direct-care-jobs. 
or robots. ${ }^{255}$ However, that position may be optimistic. The healthcare funding that supports those jobs is under serious attack ${ }^{256}$ and the drive of data-driven technologies to lower costs of labor-intensive tasks seems unrelenting. A related example from the gig economy ${ }^{257}$ are the ride-sharing services such as Uber and Lyft. These services provide several hundred thousand relatively low-paying jobs. However, they are already under threat as both companies invest heavily in self-driving "robot" vehicles. ${ }^{258}$ Noel Sharkey and Amanda Sharkey argue "there will be fewer carers available as the population ages" and that "[w[ith very advanced smart sensing systems and robots that can lift and carry, bathe and feed, as well as keep their charges safe, there will be less need for care visits. ${ }^{259}$

Just as automation may change the identity of our carers, so it may also impact the social nature of the caring experience. ${ }^{260}$ First, how much progress in the practice of caring will be made if we continue to view comfort and care as the worst jobs? Second, Sharkey and Sharkey further note the replacement of human carers with automation as, "bad news for many elderly people for whom visiting carers are the only human companionship they have on a daily basis.".261 As Frank Tobe has observed, "The many stakeholders in robotic healthcare (family members and caregivers, healthcare providers, technology providers, aging or disabled individuals) all have similar goals: To provide independence, preserve dignity, empower those with special needs and provide peace of mind to all of the stakeholders." ${ }^{262}$ Yet, those social empathetic interactions will be difficult to replicate. According to the Obama Administration's AI report,

255. Id. ("...direct care workers are not in immediate danger of being edged out by automation or internet commerce.").

256. See Danielle Paquette, The GOP Health-Care Plan Threatens to Kill Jobs Nationwide, WASH. POST (June 27, 2017), https://www.washingtonpost.com/news/wonk/wp/2017/06/27/the-gop-health-careplan-could-gut-nursing-homes-and-kill-jobs-nationwide/? (explaining how the Better Care Reconciliation Act, which is being considered by Congress, would reduce the country's Medicaid spending that could lead to the shutting down of several nursing homes).

257. See Nathan Heller, Is the Gig Economy Working?, NEW YORKER (May 15, 2017), http://www.newyorker.com/magazine/2017/05/15/is-the-gig-economy-working (explaining how the gig economy is a new model of business that allows in-app payments and gives workers more flexibility).

258. See Johana Bhuiyan, As Uber's Robot Cars Hit the Streets in Pittsburgh, the Fears of its Human Drivers . Have Become $a_{r}$ Reality, RECODE (Sept. 14, 2016, 9:10 PM), https://www.recode.net/2016/9/14/12917436/uber-self-driving-cars-pittsburgh-drivers-cars (explaining how the development of self-driving vehicles puts human drivers at risk of losing their jobs); see Andrew J. Hawkins, Lyfi is Now Building its Own Self-Driving Technology, Which is a Big Deal, VERGE (July 21, 2017, 11:00 AM), https://www.thevergc.com/2017/7/21/16005636/lyft-self-driving-car-technologyhardware-software.

259. Noel Sharkey \& Amanda Sharkey, The Rights and Wrongs of Human Care, in ROBOT ETHICS: THE ETHICAL AND Social IMPLiCATIONS OF RoBotics 267, 276 (Patrick Lin et al. eds., 2012).

260. See Lauren Smiley, What Happens When We Let Tech Care for Our Aging Parents, WIRED (Dec. 12, 2017), https://www.wired.com/story/digital-puppy-seniors-nursing-homes/.

261. $I d$.

262. Frank Tobe, Where are the Elder Care Robots?, IEEE SPECTRUM (Nov. 12, 2012), http://spectrum.icec.org/automaton/robotics/home-robots/where-are-the-eldercare-robots. 
"While AI detects patterns and creates predictions, it still cannot replicate social or general intelligence, creativity, or human judgment."263

Of course, developers will attempt to build personalities ${ }^{264}$ and even emotional intelligence into their AI constructs. ${ }^{265}$ At the University of Southern California researchers have built the SimSensei Kiosk, populated by Ellie, a virtual human possessing verbal and non-verbal cues designed to encourage human interaction in the interviews she conducts. These characteristics are particularly important because Ellie's task is to assess distress, anxiety or PTSD. ${ }^{266}$ Consider Ellie, the end-of-life bot discussed above, ${ }^{267}$ and the prototype robot Buddhist monk launched in China. ${ }^{268}$ Will programmed, even "fake" empathy, be considered a trade-off for automation's lack of "real" empathy? The issues are similar to those we must soon confront with regard to the "relationships" humans will develop with pleasure robots. ${ }^{269}$ Indeed, while much of the controversy about robotics tends to be economic, with concerns over jobs and human de-skilling, the more important issue we will have to face may be moral deskilling. ${ }^{270}$

263. Exec. Office of the President, Artificial. Intelligence, Automation, AND the 1 , 14

(2016), https://obamawhitehouse.archives.gov/sites/whitehouse.gov/files/documents/Artificial-IntelligenceAutomation-Economy.PDF.

264. See Rani Molla, Most People Prefer Friendly Robots - But Not in France and Japan, RECODE (May 20, 2017, 9:27 AM), https://www.recode.net/2017/5/20/15667494/robot-personality-customerservice-us-japan-france (reporting the results of a survey on personality types consumers would prefer be implemented in customer service robots).

265. See Geraldine Cremin, Robots Are Learning to Fake Empathy, MoTHERBOARD (Apr. 6, 2016, 7:00 AM), https://motherboard.vice.com/en_us/article/robots-are-learning-to-fake-empathy (explaining how developers at ICT are adding emotional intelligence to the AI they install in their virtual agents).

266. David DeVault et al., SimSensei Kiosk: A Virtual Human Interviewer for Healthcare Decision $\begin{array}{lllll}\text { Support, U.S.C. } & \text { FOR } & \text { CREATIVE } & \text { TECH., }\end{array}$ http://www.ccs.neu.edu/home/marsella/publications/pdf/Devault_AAMAS14.pdf (2014).

267. See supra text accompanying 104.

268. Harrict Sherwood, Robot Monk to Spread Buddhist Wisdom to the Digital Generation, GUARDIAN (Apr. 26, 2016, 9:06 AM), https:/www.theguardian.com/world/2016/apr/26/robot-monk-tospread-buddhist-wisdom-to-the-digital-generation.

269. See Jenny Kleeman, The Race to Build the World's First Sex Robot, GuARDIAN (Apr. 27, 2017, 12:30 AM), https://www.theguardian.com/technology/2017/apr/27/race-to-build-world-first-sex-robot (citing concerns that robots created by sex tech industries will create "an unnatural culture"); see also Sarah Knapton, Sex Robots on Way for Elderly and Lonely...But Pleasure-Bots Have a Dark Side, Warn Experts, TELEGRAPH (July 5, 2017, 12:01 AM), http://www.telegraph.co.uk/science/2017/07/04/sexrobots-way-elderly-lonelybut-pleasure-bots-have-dark-side/ (citing concerns about problems that may arise in use of sex robots, for example Alzheimer's patients mistaking the robots for real people).

270. See generally Shannon Vallor, Moral Deskilling and Upskilling in a New Machine Age: Reflections on the Ambiguous Future of Character, 28 PHIL. \& TECH. 107, 112 (2015) (explaining how new information and communications technologies leads to moral deskilling, the devaluation of knowledge and skillset). 


\section{B. Can Automation Handle Provider and Patient Heterogeneity?}

Healthcare is local, diverse, and personal, properties of what the late Kenneth Arrow described as healthcare's "uncertainty."271 We are continually reminded that health and healthcare problems are local. For example, health insurance markets operate differently across the country, ${ }^{272}$ the opioid crisis has differential impact across the states, ${ }^{273}$ and the ACA invested millions of dollars in patient-centered research through PCORI. ${ }^{274}$ Indeed, much of the immutability of the original triangle can be laid at the feet of patient differences. Take prescription drugs. Whatever the upside of a particular drug is, some percentage of the population will experience a quality or safety-related adverse event. ${ }^{275}$ Even more important are the cost implications - we tend to treat all patients the same, prescribing the same diagnosis-correct treatments or drugs. ${ }^{276}$ But, few patients will actually benefit. That is one of the main thrusts behind pharmacogenetics and our heavy investment in other personalized and precision medicine initiatives. ${ }^{277}$

Whether sourced in a caring clinician or mediated by pharmacogenetics, being treated as an individual is an important property of empathy. In contrast, one of the core meanings of "robotic" is the performance of serial tasks without variation. There is, therefore, a significant question as to the whether the empathy value of being treated as an individual can be transplanted to data-driven healthcare.

The different ways that a heterogeneous patient group might interface with healthcare data technologies also impacts empathy. Technology companies have successfully increased, to use the big data meme, the volume, variety and

271. See Kenneth J. Arrow, Uncertainty and the Welfare Economics of Medical Care, 53 AM. ECON. REV. 941, 951-52 (1963).

272. See, e.g., MichaEl MorRisey et al., How has ObamaCare IMPaCted STATE Health Care MARKETPLACES? (2017), https:/www.brookings.edu/research/how-has-obamacare-impacted-statehealthcare-marketplaces/ (explaining how the Affordable Care Act changed differently health care marketplaces in California, Florida, North Carolina, Michigan; and Texas).

273. See John S. Kiernan, Drug Use by State: 2017 's Problem Areas, WalletHub (May 15, 2017), https://wallethub.com/edu/drug-use-by-state/35150/\#main-findings (comparing drug use among the states based on 15 key metrics, including overdose rates and opioid prescription incidents).

274. PATIENT-CENTERED OUTCOMES RES. INST, https://www.pcori.org/about-us (last visited Sept. 10, 2017).

275. The Value of Precision Medicine, GENETIC SCI. LEARNING : CTR., http://learn.genetics.utah.edu/content/precision/value/ (last visited Sept. 10, 2017) (explaining that "[c]ven when medications are prescribed properly, certain people will have adverse drug reactions.").

276. See Dhruv S. Kazi et al., Cost-Effectiveness of Genotype-Guided and Dual Antiplatelet Therapies in Acute Coronary Syndrome, 160 ANNALS INTERNAL MED. 221, 226-27 (2014) (contrasting personalized genotype-guided therapies with cost-effective one-size-fits-all approaches).

277. See Anton Yuryev, Personalized vs. Precision Medicine, ElsEviER, (Feb. 3, 2017), https:/pharma.elsevier.com/pharma-rd/personalized-vs-precision-medicine/ (explaining how the government budget included $\$ 215$ million for $\mathrm{NIH}, \mathrm{NCI}$, and the FDA to fund precision medicine initiative). 
velocity, of data. However, they have been less successful in the words of Carly Fiorina, to "transform data into information and information into insight." 278 Automation must be responsive to the difficulty many will have in accessing the benefits of new healthcare data technologies. Under the ACA there was innovation funding for care coordination through a patient "home."279 Increasingly, data curators may need a home or some form of patient Navigator service. $^{280}$

\section{Empathy and Equity: Will Machine Age Medicine Benefit All?}

As already noted, automation raises very difficult questions about employment and wealth distribution. ${ }^{281}$ The rise of automation is likely to continue the process of economic hollowing-out ${ }^{282}$ or "spread," 283 and as already discussed the rise of healthcare automation will have its own macroeconomic impact if it reduces the demand for human custodians and carers. ${ }^{284}$ Those

278. Carly Fiorina, Address at the Oracle OpenWorld (Dec. 6, 2004) (available at http://www.hp.com/hpinfo/execteam/speeches/fiorina/04openworld.html).

279. See Patient-Centered Medical Homes, HealTH Aff. Blog (Sept. 14, 2010), http:/www.healthaffairs.org/healthpolicybriefs/brief.php?brief_id=25 (explaining that within Medicare or Medicaid, the HHS secretary has been extended the authority to further innovate patient-centered medical homes).

280. See Ana Natale-Pereira et al., The Role of Patient Navigators in Eliminating Health Disparities, 117 CANCER 3543, 3548 (2011) (explaining that patient navigators help racial/ethnic groups access resources and systems according to their needs).

281. See supra text accompanying note 116; see Yuval Noah Harari, Are We About to Witness the Most Unequal Societies in History?, GUARDIAN (May 24, 2017, 7:20 PM), https://www.theguardian.com/inequality/2017/may/24/are-we-about-to-witness-the-most-unequalsocieties-in-history-yuval-noah-harari (" $[\mathrm{B}]$ ioengineering coupled with the rise of $\mathrm{AI}$ - may result in the separation of humankind into a small class of superhumans, and a massive underclass of 'useless' people.").

282. See Andrew Soergel, 'Hollowing Out' of Middle Class Holding Back Economy, Says IMF, U.S. News (June 29, 2016, 3:34 PM), https://www.usnews.com/news/articles/2016-06-29/hollowing-out-ofmiddle-class-holding-back-economy-says-imf (discussing how income inequality has caused "hollowing out of the middle class" due to polarization in income).

283. See supra BRYNJOLFSSON \& MCAFEE note 29 at 198 (discussing how the advancement of technology will replace workers with "ordinary" skills at an extraordinary rate).

284. See supra text accompanying note 254. 
phenomena may only worsen the health disparities problem in the U.S., ${ }^{285}$ disparities based on race and ethnicity ${ }^{286}$ or income. ${ }^{287}$

Therefore, Automation must address a fundamental question-will healthcare data technologies increase or decrease healthcare disparities? As a politician foolishly remarked, "rather than getting that new iPhone that they just love and they want to go spend hundreds of dollars on, maybe [Americans] should invest it in their own health care. They've got to make those decisions for themselves." ${ }^{288}$ Hubris (and a false dichotomy) aside this does raise questions about equity and access. Some believe that presenting easily digestible information on a smartphone could be a major weapon to attack the nation's health illiteracy problem. ${ }^{289}$ However, consumer-facing technologies such as smartphones and high-end wearables (and their data plans) or health-conscious connected automobiles are not the healthcare tools of the poor or the unhealthy:

The above discussion impliedly accepts that Automation would be applied neutrally to healthcare. However, the owners of future data-driven services may not be particularly interested in the poor or the unhealthy, except perhaps to exclude them from services. Data-based decision-making may contain implicit biases; for example, by making choices solely on the basis of the data rather than taking into account other characteristics (empathy would be one) or for failing to control for the implicit biases of the programmers. ${ }^{290}$ And, as Frank Pasquale suggests, there may be only baby steps between implicit and explicit bias,

285. See generally Petry Ubri \& Samantha Artiga DisPariTies In HEALTH AND HEALTh CARE: Five KEY QUESTIONS AND ANSWERS; KAISER FAMILY 'FOUND.' $1-2$ (2016), http://files.kff.org/attachment/Issue-Brief-Disparities-in-Health-and-Health-Care-Five-Key-Questionsand-Answers (explaining that minority groups and low-income populations are at a disproportionate risk of reduced access to health care and having worse health outcomes).

286. See INST. OF MED., UNEQUAL TREATMENT: CONFRONTING RACIAL AND ETHNIC DisPaRITIES IN HEALTH CARE 5 (Brian D. Smedley et al. eds., 2003) (explaining that racial and ethnic minorities often face many barriers to accessing healthcare due to factors such as language or culture, which leads to lower quality of care)

287. See Joachim O. Hero et al., The United States Leads Other Nations in Differences by Income in Perceptions of Health and Health Care, 36 HEALTH AFF. BLOG 1032, 1034-37 (2017) (discussing how the large income difference in the United States has led to health disparities due to issues such as high outof-pocket expenses because of incomplete insurance coverages).

288. Meridith McGraw, Chaffetz Suggests Americans May Need to Choose Between iPhone and Health Care, ABC News (Mar. 7, 2017, 12:16 PM), http://abcnews.go.com/Politics/chaffetz-suggestsamericans-choose-iphone-health-care/story?id=45965471 (quoting Republican Jason Chaffetz, the chairman of the House Oversight Committee).

289. See Ahmed Albaiti, How Smartphones Can Actually be Good for Your Health, STAT (Mar." 10, 2017), https:/www.statnews.com/2017/03/10/smartphones-health-literacy/ '(describing studies shốwing that cell phones provide users with access to easily understandable and digestible information to help with the health illiteracy problem).

290. See Danny Bradbury, Sorry, Dave, I Can't Code That: AI's Prejudice Problem, REGISTER (May 8, 2017, 9:01 AM), https://www.theregister.co.uk/2017/05/08/algorithmic_bias/? (explaining that algorithms can be bias due to reasons such as the data-set used could be bias or the bias could have been deliberately coded into the algorithm). 
"Reputation systems are creating new (and largely invisible) minorities, disfavored due to error unfairness... [algorithms] are programmed by human beings, whose values are embedded into their software. And they must often use data laced with all-too-human prejudice."291

Any improvements in clinical care triggered by increased automation must also be viewed through a resource allocation lens and placed, for example, in the broader context of what factors influence length of life and quality of life. For example, it has been argued that clinical care has a relatively minor (twenty percent) compared to social determinants of health (fifty percent) and health behaviors (thirty percent). ${ }^{292}$ Microsoft founder Bill Gates has suggested ${ }^{293}$ and the EU briefly considered ${ }^{294}$ that we should tax robots or AI and use the proceeds to fund re-engineering of the workforce. As we move from a moment of near healthcare universality ${ }^{295}$ to a system that practices healthism ${ }^{296}$ and ageism would a redistribution of some sort be appropriate to guarantee equal access to the most advanced health care.

\section{HeAlthCARE Automation AND APPLying THE NEW Triangle}

Lists of how technology should improve healthcare are not hard to come by. ${ }^{297}$ As alluded to above, ${ }^{298}$ the IOM said HIT would improve safety, HIPAA's Administrative Simplification was designed to further electronic transactions to

291. Frank Pasquale, The Black Box Society: The Secret algorithms That Control MONEY AND INFORMATION 38 (2015). See generally Alex Campoloet al., AI Now 2017 Report (2017) https://assets.contentful.com/8wprhhvnpfc0/1 A9c3ZTCZa2KEYM64Wsc2a/8636557c5fb14f2b74b2be6 4c3ce0c78/_AI_Now_Institute_2017_Report_pdf

292. See, e.g., Geoffrey R. Swain, How Does Economic and Social Disadvantage Affect Health?, Focus, Fall/Winter 2016-17, at 1, 2 fig.1, http://www.irp.wisc.edu/publications/focus/pdfs/foc331a.pdf (explaining that health outcomes are often determined by health behaviors, clinical care, social and economic factors, and the physical environment as well).

293. Kevin J. Delaney, The Robot That Takes Your Job Should Pay Taxes, Says Bill Gates, QuARTZ (Feb. 17, 2017), https://qz.com/911968/bill-gates-the-robot-that-takes-your-job-should-pay-taxes/.

294. European Parliament Calls for Robot Law, Rejects Robot Tax, REUTERS (Feb. 16, 2017, 2:03 PM), http://www.reuters.com/article/us-europe-robots-lawmaking-idUSKBN15V2KM; see also Jane Wakefield, MEPS Vote on Robots' Legal Status - And if a Kill Switch is Required, BBC (Jan. 12, 2017), http://www.bbc.com/news/technology-38583360 (discussing the "new industrial" robot revolution where the European Commission once considered adding regulations to regulate human robot interaction).

295. See Nicole Hubcrfeld, The Universality of Medicaid at Fifty, 15 YALE J. HEALTH POL'Y L. \& ETHICS 67, 68-69 (2015) (discussing how the Affordable Care Act shifted the law of healthcare to federally-based public law and created universal access to health insurance).

296. See Jessica L. Roberts \& Elizabeth Wecks Leonard, What is (and Isn't) Healthism?, 50 U. GA. L. REV. 833, 835 (2016) (discussing the shift towards a system that practices healthism, "discrimination on the basis of health status").

297. See, e.g., Booz Allen Lists Top Nine Ways Information Technology Is Transforming Health Care, Bus. WIRE (Nov. 15, 2011, $11: 31$ AM), http:/www.businesswire.com/news/home/20111115006639/en/Booz-Allen-Lists-Top-WaysInformation-Technology.

298. See supra text accompanying notes 11-12. 
reduce administrative costs, HITECH's Meaningful Use and its EHRs would improve clinical care and improve care coordination, Big Data Analytics would help OIG root out fraud and hospitals cut readmission rates, and so on, ad infinitum:

In this section I offer two examples of automation that is changing or will likely have a major impact on healthcare. They are Appification and AI Diagnosis, and I use them as case studies applying the new triangle.

\section{A. Appification}

The medical app ecosystem is well known, ubiquitous, and it's regulatory issues extensively documented. ${ }^{299}$ Mobile platforms (phones, smartwatches, skin sensors, and other wearables) include a multitude of healthcare-relevant sensors, including those for proximity, ambient light, and pressure, together with" cameras, accelerometers, gyroscopes, compasses, and barometers. Built on these platforms are apps that measure activity goals, help manage diabetes, monitor blood pressure, track sleep cycles, quantify activity, track stress, listen to fetal heartbeats, test vision, track fertility, examine skin imperfections... the list is endless! Mobile medical or health apps share a basic characteristic with other apps. Compared to traditional (and, usually, complicated) computer platforms or operating systems, app-based systems simplify the user experience by shifting the user experience within the interface to narrower, even isolated functions or transactions. ${ }^{300}$ Essentially the user experience is shifted from complex, multifaceted applications to simplified, highly focused ones. The user experience is "appified."

This changed experience extends beyond the mere disaggregation of computer tasks and impacts the level of abstraction. Grossly oversimplified; the lower the level of abstraction the more computing layers (hardware, software, user interface, etc.) are exposed. For example, MS-DOS and MacOS Terminal use command line interfaces that essentially feature machine language conversations between computer and user. Most users prefer interfaces that hide these layers, that have a higher level of abstraction such as Microsoft Windows or the MacOS Aqua interface. Clicking on an application in a graphical user interface typically opens the application and exposes the user to menus of actions

299. See, e.g., Cortez et al., supra note 1, at 372 (discussing the FDA regulation of mobile health technologies to ensure safety and effectiveness); see also Cortez, supra note 1. at 181; see also Terry, supra note 1, at 146 47see also Cortez, supra note 41, at 1181,1199; see also Terty, supra note 46, at 1430-33; see also Terry \& Wiley, supra note 46, at 63-64.

300. See Managing THE Growing "Appification" of Business, ACCEnTure 3 (2013), https://www.accenture.com/t20150523T130138_w_/be-en/acnmedia/Accenture/Conversion-

Assets/DotCom/Documents/Global/PDF/Technology_6/Accenture-13-3975-Appification-POV.pdf ; see also Monica Mehta, 5 Best Practices in Application User Experience, ForBES (Nov. 12, 2015, 6:15 AM), https://www.forbes.com/sites/oracle/2015/11/12/5-best-practices-in-application-userexperience//26b48d5a3824. 
and properties. Although the computer's basic systems are hidden by those interfaces, users still have to select among the application's functions and deal with issues such as where to store data generate by the application. In contrast, touching an app icon in, say iOS or Android involves far more abstraction; typically, an app will be launched that in most cases will perform one or at most only a handful of tasks (the use of the diminutive is not an accident) and the user will not be expected to make many complex decisions (e.g., the file system is hidden from the end user). ${ }^{301}$

Appification doubles as a useful metaphor for understanding contemporary attempts to disaggregate healthcare. Disaggregation efforts can be seen both inside and outside of conventional healthcare. Examples from the former would include "retail medicine" such as walk-in clinics or urgent care centers. The latter feature direct-to-consumer products (DTC), such as diabetes monitoring kits and pregnancy tests. These are all fragments of healthcare broken away from the monolith. Toby Cosgrove, CEO of the Cleveland Clinic, sees the hospital of the future built around local and remote monitoring-"When I think of the hospital of the future, I think of a bunch of people sitting in a room full of screens and phones." 302

Much of this fragmentation has been designed to placate the original triangle by offering better access at a similarly or slightly lower cost and with a minimal hit on quality. DTC products and decentralized clinics shield the patient from healthcare complexity. Rather than making an appointment at a centralized and complex healthcare entity, travelling there, waiting, being seen by several clinicians-interrupted by more wait times - receiving a prescription to be filled in yet another location, etc., the patient with a sore throat metaphorically "clicks" on a conveniently located retail clinic that can diagnose and prescribe for strep throat.

However, it is the DTC market, one not controlled by the professional distribution stack $^{303}$ and one that includes medical apps, that is the most interesting. Bill Sage and Kelley Mcllhattan have described the dividing line between upstream and downstream healthcare as the point at which a person becomes a patient. They note how our system deploys most of its resources to downstream healthcare and argue that "right-sizing health care" must include

301. See generally Gcorge Kassabgi, A Look at the Evolution of the User Interface in Healthcare, MEDCITY NEWS (Apr. 7, 2017, 12:37 PM), http://medcitynews.com/2017/04/user-interfaces-inhealthcare/ (discussing how applications appeal to a broader population since they have become more user fricndly).

302. How Hospitals Could Be Rebuilt, Better Than Before, ECONOMIST (Apr. 8, 2017), https:/www.economist.com/news/international/21720278-technology-could-revolutionise-way-theywork-how-hospitals-could-be-rebuilt-better.

303. See supra text accompanying note 182 . 
"buy[ing] health without becoming a patient." 304 Further, in the words of Kevin Maney, "As startups bring these technologies online, they're often doing an end run around insurance companies, instead finding demand among consumers or employers who offer health coverage. ${ }^{.305}$

Medical apps have the potential to do much more than tallying footsteps. Many also disaggregate the complexity associated with traditional healthcare. They peel off various aspects of the healthcare system, frequently aspects that should be made available upstream, and present them in exceptionally easy-touse software. The most impressive contemporary impressive apps operate downstream, monitoring patients in their homes and managing diagnosed chronic conditions. ${ }^{306}$. However, driven by AI-based analytics, pre-diagnosis, upstream apps that diagnose and monitor physical and mental health will improve rapidly.

Assuming that rise in automation, how will appification withstand scrutiny from the other points of the new triangle, value and empathy? There is some research that suggests health apps are simply this generation's snake oil; electronic multivitamin supplements that are a scam or, at least, a waste of money. Certainly, there is justifiable skepticism about wearable devices and their claims to promote fitness and weight loss. ${ }^{307}$ For example, most weärables are relatively poor at measuring key vital signs. ${ }^{308}$

304. William M. Sage \& Kelley Mcllhattan, Upstream Health Law, 42 J. L. MED. \& ETHICS 535, 53536 (2014).

305. Kevin Maney, How Artificial Intelligence Will Cure America's Sick Health Care System, NEwSWEEK (May 24, 2017, 9:27 AM), http://www.newsweek.com/2017/06/02/ai-cure-america-sickhealth-care-system-614583.html.

306. See, e.g., Laura Landro, How Apps Can Help Manage Chronic Diseases, WaLL St. J. (June 25, 2017, 10:12 PM), https://www.wsj.com/articles/how-apps-can-help-manage-chronic-diseases1498443120 (explaining that digital medicine can now help paticnts manage their costly and hard to manage chronic conditions, such as lung disease); see also Heather Mack, FDA Clears Duo, A Combination Smart Heart Monitor and Stethoscope from Eko, MoBIHEALTHNEws (June 7, 2017), 'http://www.mobihealthnews.com/content/fda-clears-duo-combination-smart-heart-monitor-andstethoscope-eko (noting that new devices can help avoid episodic care and allows for continuous patient monitoring, especially for patients with complex conditions).

$\because$ 307. See Travis M. Andrews, Fitness Trackers Are Largely Inaccurate When Counting Calories, Stanford Researchers Say, WASH. POST (May 25, 2017), https:/www.washingtonpost.com/news/morning-mix/wp/2017/05/25/fitness-trackers-are- ' largelyinaccurate-when-counting-calories-stanford-researchers-say/? (discussing the accuracy of fitness trackers and implications for health); see also Aaron E. Carroll, Wearable Fitness Devices Don't Seem to Make You Fitter, N.Y. TiMEs (Feb. 20, 2017), https:/www.nytimes.com/2017/02/20/upshot/wearable-fitnessdevices-dont-seem-to-make-you-more-fit.html? (explaining that cvidence does not support the claim that wearable fitness devices make the wearer more fit).

308. See Katic Glenn, Wrist-Worn Heart Rate Monitors Less Accurate than Standard Chest Strap, AMERICAN COLL. OF CARDIOLOGY (Mar. 8, 2017), http://www.acc.org/about-acc/pressreleases/2017/03/08/14/02/wrist-worn-heart-rate-monitors-less-accurate-than-standard-chest-strap (discussing a study comparing fitness trackers which determined that chest strap monitors provided optimal data). 
There is little traditional value regulation of upstream mobile apps, notwithstanding the serious questions raised about data protection, safety, and cost-effectiveness. HHS essentially admitted that most apps operate outside of the HIPAA protected zone, ${ }^{309}$ while the FDA has exercised considerable discretion in a risk-based sub-regulatory guidance. ${ }^{310}$ Meanwhile, both the FTC and the guardians of the Apple app store seem to be playing a long game of whack-a-mole when it comes to weeding out the useless and the fraudulent. ${ }^{311}$ Thus, the obvious regulatory route for resolving or at least ameliorating the automation-value tension is missing in action. One forthcoming tension does seem to be clear; the power and usefulness of apps increases considerably when data storage and processing is off-loaded to cloud-based systems, but that also increases the likelihood of privacy and security breaches compared to onboard storage and processing. ${ }^{312}$ This traditional automation-value therefore may be in flux. The autonomy-based strict liability position of HIPAA and the breach of confidence tort that heretofore have dominated the interaction of man and medicine may see a renewed instrumental attack during the time of healthcare data technologies. For example, Ignacio Cofone has proposed embedding privacy concerns in QALY-based CEA. ${ }^{313}$

The automation-empathy tension regarding Appification is less clear-cut. The first generation of health-related apps have concentrated on selfquantification. There is research suggesting that quantifying enjoyable life activities undermines intrinsic motivation and makes the activities seem "more like work," 314 "[r]educing daily experience to a series of boxes checked and numerical goals met colonize consumers' leisure time with the same disciplined logic of their working days." 315 These tendencies will likely increase as employers and life insurers create pressure through, for example, wellness

309. HITPC PRIVACy \& SEC. WORkgRoup, HEAlTh Big Data ReCOMMEndations 4 (2015), https://www.healthit.gov/sites/faca/files/HITPC_Health_Big_Data_Report_FINAL.pdf.

310. 2015 FDA MOBILE MEdICAL APPLICATIONS: GUIDANCE FOR INDUSTRY AND FOOD AND DRUG ADMINISTRATION STAFF, supra note 163, at 4, 15-18; 2017 FDA DIGITAL HEALTH INNOVATION ACTION PLAN, supra note 171, at 5-6.

311. See supra notes $175,230-31$ and accompanying text.

312. See, e.g., Tom Simonite, Apple's Privacy Pledge Complicates Its AI Push, WIRED (July 14, 2017, 9:00 AM), https://www.wired.com/story/apple-ai-privacy/ (describing the increased risk of privacy breaches as the cost for utilizing efficient cloud-based storage models).

313. Ignacio N. Cofone, A Healthy Amount of Privacy: Quantifying Privacy Concerns in Medicine, 65 CLEV. ST. L. REV. 1, 12, 14 (2016) (proposing to include privacy in the costs of health treatments or include privacy concerns as a moderating element in the benefit section of ICER evaluations, within the QALYs).

314. Jordan Etkin, The Hidden Cost of Personal Quantification, 42 J. CONSUMER RES. 967, 969 (2016).

315. Frank Pasquale, How Fitness Trackers Make Leisure More Like Work, ATLANTiC (Mar. 2, 2016), http://www.theatlantic.com/business/archive/2016/03/how-trackers-make-leisure-like-work/471864/ (describing a study that found exercise tracking applications reduced user enjoyment of the exercise). 
plans ${ }^{316}$ or insurance premium discounts designed to promote the use of fitness devices. ${ }^{317}$ The question may quickly evolve into how and where we can be forced to use trackers ${ }^{318}$ and what limitations apply to the use of data they collect. ${ }^{319}$

Those empathy-based objections may not carry through to next-generation apps. Some studies suggest that consumers already prefer, even trust some apps more than their real-world physicians. ${ }^{320}$ Intuitively, that seems plausible. Recently, when planning a journey how many of us have gone to a bookstore to buy a map or visited AAA to get a TripTik? ${ }^{321}$ When lost, how many of us still stop to ask a nearby pedestrian for directions? Of course not, why would we indulge in such human interactions when we can simply click on Waze or Apple Maps? Instrumental reasoning potentially will reduce empathy-based criticisms of apps and appification in the same way that convenience has tended to lessenother values-based concerns such as privacy. This is increasingly likely as next generation apps introduce sophisticated diagnostics and condition-monitoring.

Notwithstanding, reduced appification-empathy tension may be short-lived as apps become less about layering convenience on top of conventional patientprovider relationships and increasingly data-driven. Further, as apps (and other

316. See James A. Martin, Pros and Cons of Using Fitness Trackers for Employee Wellness, CIO (Mar. 24, 2014, 8:00 AM), https:/www.cio.com/article/2377723/it-strategy/pros-and-cons-of-usingfitness-trackers-for-employee-wellness.html (describing employer authority to approve benefits as leverage to pressure employees into health and fitness tracking programs).

317. See, e.g., Christina Farr, Weighing Privacy vs. Rewards of Letting Insurers Track Your Fitness,

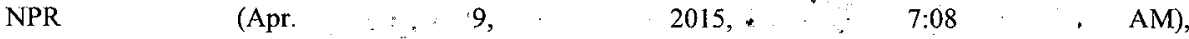
http:/www.npr.org/blogs/alltechconsidered/2015/04/09/398416513/weighing-privacy-vs-rewards-ofletting- insurers-track-your-fitness (stating that insurance company, John Hancock, offers a voluntary program where fit customers may exchange activity data for lower insurance premiums).

318. See Suzanne McGee, How Employers Tracking Your Health Can Cross the Line and Become Big Brother, GUARDIAN (May 1, 2015, 8:30 AM), https://www.theguardian.com/lifeandstyle/us-moneyblog/2015/may/01/employers-tracking-health-fitbit-apple-watch-big-brother (discussing the manner in which employers use wearable devices to implement wellness plans and the impact on employees).

319. See Patience Haggin, As Wearables in Workplace Spread, So Do Legal Concerns, WALL ST. J. (Mar. 13, 2016, 10:12 PM), https://www.wsj.com/articles/as-wearables-in-workplace-spread-so-do-legalconcerns-1457921550 (discussing growing trend of employer fitness track and employer efforts to receive only anonymized data, avoiding privacy challenges); see also Jocelyn Samuels, How HIPAA Applies to Certain Workplace Wellness Programs, U.S. Dep'T OF HeAlTH \& HuMAN SERvs. (Mar. 14, 2016), https://wayback.archive-it.org/8315/201701 19093303/https://ww.hhs.gov/blog/2016/03/14/how-hipaaapplies-certain-workplace-wellness-programs.html\# ("While the Health Insurance Portability and Accountability Act (HIPAA) does not apply to all workplace wellness programs, it does apply to programs offered as part of an employer-sponsored group health plan.").

320. See generally Jennifer Elias, In 2016, Users Will Trust Health Apps More Than Their Doctors, FORBES (Dec. 31, 2015, 6:00 AM), https:/www.forbes.com/sites/jenniferelias/2015/12/31/in-2016-userswill-trust-health-apps-more-than-their-doctors/\#6a0657777cb6 (describing multiple surveys that address trends in digital health management, mobile health and fitness application use and the impact of applications on the traditional patient-doctor relationship).

321. AAA Travel Information, AM. AUTO. ASS'N, http:/www.aaa.com/travelinfo/maps-directions.htm (last visited Sept. 7, 2017). 
AI healthcare data technologies) become increasingly autonomous questions abound as to both the possibility and the desirability of programming ethical frameworks (aka artificial moral agents) into machines. ${ }^{322}$ The kind of challenge faced by programmers is illustrated by the "Trolley Problem," a well-known thought experiment that addresses how actors react to the relative worth of persons when a technology threatens life or serious injuries. ${ }^{323}$

\section{B. AI Diagnostics}

While it is unlikely that robots or AI will take over all of healthcare in, say, the next two or three decades, it is far more probable that AI will have primary responsibility for at least some medical tasks in that same timeframe. Just as disaggregation flows into real and metaphorical appification, so some components of healthcare likely will be peeled off and given over to the machines.

Not surprisingly, AI is being tested or used in various healthcare settings and against a variety of tasks. For example, it is being used to create smarter decision support environments, ${ }^{324}$ classify skin cancers, ${ }^{325}$ the VA has been testing IBM's Watson to determine its worth in searching the clinical literature and patient records to generate a patient "problem list," 326 Alphabet's Deep Mind is working with the Royal Free Hospital in London on a system that uses AI to alert clinicians about acute kidney disease in their patients, ${ }^{327}$ and Boston-area

322. See generally Colin Allen \& Wendell Wallach, Moral Machines: Contradiction in Terms or Abdication of Human Responsibility, in ROBOT ETHICS: THE ETHICAL AND SOCIAL IMPLICATIONS OF RовотіCS 55 (Patrick Lin et al. eds., 2011) (arguing for the need of increasingly autonomous systems that have the capabilities of factoring ethical and moral considerations into the decision-making process).

323. See generally Sarah Bakewell, Clang Went the Trolley, N.Y. TIMES (Nov. 22, 2013), http:/www.nytimes.com/2013/11/24/books/review/would-you-kill-the-fat-man-and-the-trolleyproblem.html (explaining the moral dilemma of whether to save five people on a trolley by diverting the trolley from its current collision course, but killing one person tied to the tracks); see also Patrick Lin, The Ethics of Autonomous Cars, ATLANTIC (Oct. 8, 2013), http://www.theatlantic.com/technology/archive/2013/10/the-ethics-of-autonomous-cars/280360/ (applying the trolley moral dilemma to the development of advanced sensors and programming for autonomous cars).

324. See, e.g., Thomas H. Davenport \& John Glaser, Just-In-Time Delivery Comes to Knowledge Management, HARV. BUS. REV. (July 2002), https://hbr.org/2002/07/just-in-time-delivery-comes-toknowledge-management (describing the development of integrated information systems that reduce medical errors by offering medical records systems at time of diagnosis).

325. See Andre Esteva et al., Dermatologist-Level Classification of Skin Cancer with Deep Neural Networks, 542 NATURE 115, 115 (2017) (utilizing convolutional neural networks for the classification of skin cancers). See also Dom Galcon, AI-Assisted Detection Identifies Colon Cancer Automatically and in Real-Time, FUTURISM (Oct. 30, 2017), https://futurism.com/ai-assisted-detection-identifies-colon-cancerautomatically-and-in-real-time/.

326. Nicole Blake Johnson, 5 Facts About the VA's IBM Watson Pilot, FedTECH (Jan. 21, 2015), http://www.fedtechmagazine.com/article/2015/01/5-facts-about-va-s-ibm-watson-pilot.

327. James Temperton, DeepMind Hits Back at Criticism of its NHS Data-Sharing Deal, WIRED (Nov. 22, 2016), http://www.wired.co.uk/article/deepmind-nhs-data-sharing-streams-app-privacy (describing 
hospitals are testing machine-learning algorithms that are vastly improving early predictions of diabetes and heart disease. ${ }^{328}$

The question is, where will AI make its first major impression on what heretofore has been viewed as traditional, physician-centric healthcare? According to Vinod Khosla, "Much of what physicians do (checkups, testing, diagnosis, prescription, behavior modification, etc.) can be done better by sensors, passive and active data collection, and analytics. ${ }^{329}$ Indeed, the next generation apps and their platforms discussed above are part of this data collection apparatchik for the automated systems of our near future. Increasingly (and perhaps less transparently) the Io $T$ bots and carer robots surrounding humans will collect immense quantities of data. ${ }^{330}$ Khosla continues, "doctors aren't supposed to just measure... [but also] consume all that data, consider it in context of the latest medical findings and the patient's 'history, and figure out if something's wrong." 331

. The focus here on diagnostics is not only because of a contended-for good "fit" with automation's data-gathering and data processing, ${ }^{332}$ but also because improving diagnostics is an important policy goal. According to the IOM, "5 percent of U.S. adults who seek outpatient care each year experience a diagnostic error" and that such errors account for 6 to 17 percent of adverse events in hospitals." 333 The IOM went so far as to describe diagnostic errors as a decadeslong "blind spot." 334 To give one specific example, a recent clinical report that followed a single patient's lumbar MRI results in 10 different MRI centers over a three-week period. The disparity between diagnoses was marked with a missrate of $43.6 \% .^{335}$ Diagnostic errors also are responsible for the largest percentage

the application Streams, which uses artificial intelligence to send physicians real-time alerts about acute kidney disease).

328. See Yannis Paschalidis, How Machine Learning is Helping Us Predict Heart Disease and Diabetes, HARV. BuS. REV. (May 30, 2017), https://hbr.org/2017/05/how-machine-learning-is-helpingus-predict-heart-disease-and-diabetes (analyzing anonymized electronic health records of Boston area hospitals to predict future hospitalization with $82 \%$ accuracy).

329. Khosla, supra note 34.

330. Simshaw et al., supra note 103, at 11-15 (describing the increased use of autonomous robotic functions in providing medical assistance).

331. Khosla, supra note 34.

332. See, e.g., AI has Surpassed Cardiologists in Predicting the Heart Attack Efficiency, STARTLR (Apr. 18, 2017), http:/www.startlr.com/ai-has-surpassed-cardiologists-in-predicting-the-heart-attackefficiency/ (describing the use of artificial intelligence to predict the likelihood of a heart attack with $72.8 \%$ accuracy).

333. Committee on Diagnostic Error in Health Care, InSt. Of MED., Improving Diagnosis IN HEALTH CARE (Erin P. Balogh et al. eds., 2015) (ebook); see Hardeep Singh et al., The Frequency of Diagnostic Errors in Outpatient Care: Estimations From Three Large Observational Studies Involving U.S. Adult Populations, 23 BMJ QuAliTY \& SAFETY 727, 727-30 (2014) (combining data sources to determine the rate of diagnostic errors in healthcarc).

334. COMmitTEe ON Diagnostic ERROR IN HEALTH CARE, supra note 333 at 19.

335. Richard Herzog et al., Variability in Diagnostic Error Rates of 10 MRI Centers Performing Lumbar Spine MRI Examinations on the Same Patient Within a 3-Week Period, 17 SPINE J., 554, 559 
of medical malpractice paid claims both as to error type $(28.6 \%)$ and total payments $(35.2 \%){ }^{336}$

AI is not the only automation in play here. Clinical decision support systems have been focused on the diagnostic problem ${ }^{337}$ and energy directed at creating much smarter decision support environments with full physician participation. ${ }^{338}$ Unfortunately, according to AHRQ, "reliable decision support or feedback systems do not yet exist." 339 Indeed, "computerized diagnostic decision support has not yet been proven to improve overall diagnostic accuracy, although active research continues in this area." ${ }^{340}$ Almost inevitably, decision support systems as we know them will cede ground to AI systems. Increasingly, clinical decision support systems will shift from programmed existing knowledge rule-based expert systems to data-mining-based systems that rely on pattern recognition and advanced algorithms. ${ }^{341}$

Recently, the HumanDX project has approached the diagnosis gap by combining a professional crowdsourcing model with fledgling $\mathrm{AI}^{342}$ Such a hybrid approach is attractive. After all, according to Brynjolfsson \& McAfee, note "creating a machine-based system for covering all possible medical cases is

(2017), http://www.thespinejournalonline.com/article/S1529-9430(16)31093-2/pdf; see generally Monica Van Such et al., Extent of Diagnostic Agreement Among Medical Referrals, 23 J. EvALUATION ClinICAL PraC., 870, 870-72 (2017), http://onlinelibrary.wiley.com/doi/10.1111/jep.12747/epdf (discussing diagnostic uncertainty through a study comparing levels of consistency in medical diagnoses).

336. Ali S. Saber Tehrani et al, 25-Year Summary of U.S. Malpractice Claims for Diagnostic Errors 1986-2010: An Analysis From the National Practitioner Data Bank, 22 BMJ QUALITY \& SAFETY 672, 676 (2013).

337. See Alejandro Rodríguez-González et al., Analysis of a Multilevel Diagnosis Decision Support System and its Implications: $A$ Case Study, 2012 COMPUTATIONAL \& MATHEMATICAL METHODS IN MED. 1, 2 (2012), https://www.hindawi.com/journals/cmmm/2012/367345/ (describing the use of multilevel diagnosis support systems to provide diagnoses in unfavorable conditions).

338. See Davenport \& Glaser, supra note 324 (highlighting that the key to success is baking specialized knowledge into the jobs of highly skilled workers to the extent that the knowledge is unavoidable); see generally Andrew Q. Ninh, DocBot: A Novel Clinical Decision Support Algorithm, ENGINEERING IN MEDICINE \& BIOLOGY SOC'Y 6290 (2014) (explaining that DoctBot is a web-based system that uses patient interaction and electronic health record analytics to assist medical practitioners with decision making).

339. Diagnostic Errors within Primers, PATIENT SAFETY NETWORK, https://psnet.ahrq.gov/primers/primer/12/diagnostic-errors (last updated June 2017).

340. Id.

341. See generally Bunyamin Ozaydin et al., Data Mining and Clinical Decision Support Systems, in Clinical Decision Support Systems: Theory and Practice 45, 46-48 (Eta S. Berner ed., 3d ed. 2013) (stating that the decision maker must provide facts and rules to the inference engine in rule-based expert decision support, while decision support systems that employ data mining tools do not require such a priori knowledge.).

342. Project Background, HUMANDX, https://www.humandx.org/context/background (last visited Nov. 12, 2017); see also Megan Molteni, Want a Diagnosis Tomorrow, Not Next Year? Turn to AI, WIRED (Aug. 10, 2017, 12:00 AM), https://www.wired.com/story/ai-that-will-crowdsource-your-next-diagnosis/ (explaining the evolution and potential ramifications of the HumanDX project as it bridges the gap between traditional healthcare and $\mathrm{AI}$ ). 
radically more difficult than building one for the most common situations," leading to the suggestion that "a partnership between Dr. Watson and a human doctor will be far more creative and robust than either of them working alone." 343 The more difficult question is whether such hybrid systems will end up being merely an interim step on the way to machine-based diagnostics for all, or at least most, conditions. For example, the U.K. startup Babylon Health is trialing an AIpowered chatbot 'triage' service that is edging away from human participation. ${ }^{344}$ Thus, human-based or human-participating diagnostic models could be chasing a fast receding target. According to Khosla, "Most doctors couldn't possibly read and digest all of the latest 5,000 research articles on heart disease. And, most of the average doctor's medical knowledge is from when they were in medical school, while cognitive limitations prevent them from remembering the $10,000+$ diseases humans can get. ${ }^{3345}$ In contrast, according to doctors at the University of Tokyo IBM Watson recently diagnosed a rare form of leukemia that had been missed by the treatment team. ${ }^{346}$ Further, it should be no surprise that Google already has a patent for automated diagnosis. ${ }^{347}$

How will the new triangle treat AI-diagnostics? Specifically, what tensions may arise between this type of Automation, Value, and Empathy? According to Pedro Domingos in The Master Algorithm, if you expose a database of patient records to the Naïve Bayes algorithm ${ }^{348}$ it "can learn to diagnose the condition in a fraction of a second, often better than doctors who spent many years in medical school. It can also beat medical expert systems that took thousands of person-hours to build." ${ }^{349}$ If this proves to be correct and given that we define Value primarily in terms of risk-benefit analysis then, in the long-term there should be few major tensions between automation and value.

343. BRYNJOLFSSON \& MCAFEE, supra note 29 , at ch. 12.

344. See Steve O'Hear, Babylon Health Raises Further $\$ 60 M$ to Continue Building Out AI Doctor App, TEChCRUNCH (Apr. 25, 2017) https://techcrunch.com/2017/04/25/babylon-health-raises-further$60 \mathrm{~m}$-to-continue-building-out-ai-doctor-app/ (discussing Babylon Health's proposed build out of AI technology to include diagnosis in addition to the more simple triage function).

345. Khosla, supra note 34.

346. Bernie Monegain, IBM Watson Pinpoints Rare Form of Leukemia After Doctors Misdiagnosed Patient, HealthCARE IT News (Aug. 8, 2016, 10:58 AM), http://www.healthcareitnews.com/news/ibmwatson-pinpoints-rare-form-leukemia-after-doctors-misdiagnosed-patient.

347. See generally Method \& Apparatus for Automated Med. Diagnosis Using Decision Tree Analysis, U.S. Patent No. 4,733,354 (filed Nov. 23, 1984) (claiming an interactive computer system for performing dermatological biopsy); see also John Timmer, Given Enough Training Images, Computers Compete with Medics on Diagnosis, ARS TECHNICA (Jan. 25, 2017, 2:40 PM), https://arstechnica.com/science/2017/01/deep-neural-network-as-good-as-medics-at-identifying-skincancers/ (highlighting the latest example of the effect of neural networks within computers on the area of medical diagnosis).

348. Domingos, supra note 113, at 23; see also Eliezer S. Yudkowsky, An Intuitive Explanation of Bayes' Theorem, http://yudkowsky.net/rational/bayes (last updated June 4, 2006) (explaining the application and practicality of the Bayes algorithm).

349. DOMINGOS, supra note 113 , at 23. 
In the shorter term, and particularly during the transition from human to automated diagnosis, there may be significantly more value-based reasons for concern. Some of these issues likely will mirror contemporary problems with clinical decision support systems. For example, a physician receiving an AIdelivered diagnosis might ignore it or disregard it the recommendation. ${ }^{350}$ Equally, such an alert might get buried in the large number of alerts. ${ }^{351}$ More fundamentally, early AI-diagnostic systems may make serious mistakes. Such failures would raise questions about the potential liability of healthcare providers or technology developers. ${ }^{352} \mathrm{~A}$ conspicuous failure rate could also lead to device regulatory reaction. Recall, for example, early cases of accidents involving autonomous vehicles. ${ }^{353}$ Regulators could have repeated the over-regulation mistake of the nineteenth century English Locomotive Acts that required a man carrying a red flag to walk in front of large road vehicles. Another regulatory approach was a testing ban, ${ }^{354}$ while a far more moderate approach was to recalibrate the default relationship between driver and vehicle. 355

A related question is how those who regulate the professions will react to AI diagnostics. The "professional distribution stack" and the untimely demise of the Sedasys anesthesiology machine should be a lesson here. ${ }^{356}$ Will state licensing bodies cede regulation of AI "doctors" to the FDA or will they pursue their own regulation based on the "practice of medicine" $?^{357}$ Consider, for

350. See, e.g., A.E. Carroll et. al, Understanding Why Clinicians answer or Ignore Clinical Decision Support Prompts, 3 APP. CLIN. INFORM. 309, 312 (Aug. 01, 2012).

351. Alert Fatigue within Primers, PATIENT SAFETY NETWORK, https:/psnet.ahrq.gov/primers/primer/28/alert-fatigue (last updated June 2017).

352. See generally Terry \& Wiley, supra note 46 (postulating the circumstances and scenarios which may lead to physicians being held liable as a result of using AI-diagnostic systems); see generally Nicolas Terry, When the Machine That Goes 'Ping' Causes Harm: Default Torts Rules and TechnologicallyMediated Health Care Injuries, 46 ST. LoUIS L.J. 37 (2002) (arguing that AI-diagnostic systems will inevitably test our current torts operational rules, especially as it pertains to the intersection between malpractice and products liability).

353. See, e.g., Brian Fung, The Driver Who Died in a Tesla Crash Using Autopilot Ignored at Least 7 Safety Warnings, WASH. POST (June 20, 2017), https://www.washingtonpost.com/news/theswitch/wp/2017/06/20/the-driver-who-died-in-a-tesla-crash-using-autopilot-ignored-7-safety-warnings/ (reporting the world's first known car crash involving an autonomous vehicle).

354. Cecille De Jesus, Following Tesla Crash, China Bans the Testing of Autonomous Cars on Highways, FUTURISM (July 21, 2016), https://futurism.com/following-tesla-crash-china-bans-the-testingof-autonomous-cars-on-highways/ (explaining China's ban on testing self-driving cars so that legislation can catch up with the emerging technology).

355. See Gene, Tesla Autopilot v8.0 Restrictions: Three Strikes and You're Out, TeSLARATI (Sept. 26, 2016), https:/www.teslarati.com/tesla-autopilot-version-8-0-nags-restrictions/ (explaining how Tesla drivers will need to abide by a series of beeps in order to keep the vehicle in autopilot, ensuring that drivers remain alert at all times).

356. See Sarah Fecht, Once-Promising Robot Anesthesiologist Loses its Job, PopulaR SCI. (Mar. 29, 2016), https://www.popsci.com/anesthesiology-robot-gets-put-to-sleep (cxplaining how human anesthesiologists never welcomed the Sedasys anesthesiology machine, leading to its demise).

357. What is a State Medical Board?, FED'N OF STATE MED. BDS., https://www.fsmb.org/policy/consumer-resources/frequent-questions (last visited Nov. 15, 2017) 
example, how the medical profession reacted to physician extenders such as nurse practitioners (NPs) or physician assistants (PAs). Today, the scope of practice of NPs varies depending on jurisdiction. ${ }^{358}$ Some jurisdictions allow NPs to diagnose and treat without any involvement from a physician. Others require different levels of physician involvement, from working within protocols or having samples of their work reviewed. Still other variants apply to prescribing by NPs. ${ }^{359}$ Not only would such steps demonstrate some tensions within Value, but considerable tension between Automation and Value. At a time of great technological change and potential professional upheaval making a doctor "carry a red flag" in front of every AI diagnostic machine would be unhelpful. Nevertheless, a more moderate regulatory system could be imagined and AI diagnostics mediated by human clinicians would be consistent with Kevin Keliy's observation, "This is not a race against the machines. If we race againstthem, we lose. This is a race with the machines. You'll be paid in the future based on how well you work with robots." ${ }^{~} 60$ However, another race is already underway; whether healthcare providers learn to become technology-driven (even technology) companies) "more quickly than the technology companies learn how provide healthcare?"361 If technology companies, unencumbered by innovation-sapping third-party reimbursement and recurring patterns of building

(explaining that state medical boards have the authority to license and regulate physicians within the medical field); see, e.g., WASH. REV. CODE $\$ 18.71 .011$ (2011) (defining the "practice of medicine").

358. See generally Nurse Practitioner Scope of Practice Laws, KAISER FAMILY Found., http://kff.org/other/state-indicator/total-nurse-

practitioners/?currentTimeframe=0\&sortModel $=\% 7 \mathrm{~B} \% 22$ colld $\% 22: \% 22$ Location $\% 22, \% 22$ sort $\% 22: \% 2$ 2asc $\% 22 \% 7 \mathrm{D}$ (last visited Nov. 10, 2017) (highlighting the jurisdictional differences in the level of independent involvement between nurse practitioners and patients); see also Updated Nurse Practitioner Scope of Practice Map, Ctr. FOR PUB. HEALTH L. REV. (July 19, 2016), http://blogs.harvard.edu/billofhealth/2016/07/19/updated-nurse-practitioners-scope-of-practice-map/ ( identifying states that provide full practice authority to nurse practitioners and those that provide a more limited practice authority).

359. See, e.g., Practice ReQUirements for Nurse Practitioners; N.Y: State Edúc. DeP'T, OFFICE OF THE PROFESSIONS, http://www.op.nysed.gov/prof/nurse/np-prfnp.pdf (last visited Nov. 17, 2017) (highlighting some additional criteria for some practicing nurse practitioners, such as collaboration with physicians and written practice agreements).

360. Kelly, supra note 25.

361. This is a modification of "the big question is always, Do we car manufacturers learn to become tech companies more quickly than a tech company learns to be an automotive player?" Jack Ewing \& Mark Scott, German Automakers Step Up to Silicon Valley Challenge, N.Y. TIMES (Feb. 8, 2017), https://www.nytimes.com/2017/02/08/business/germany-bmw-daimler-volkswagen-uber.html (addressing the inter-connectivity and overlap between car manufacturers and tech companies). A similar sentiment has been addressed to the competition between online and brick-and-mortar stores. See Sarah Perez, Amazon Wants to Become Walmart Before Walmart Can Become Amazon, TECHCRUNCH (June 17, 2017), https://techcrunch.com/2017/06/16/amazon-wants-to-become-walmart-before-walmart-canbecome-amazon/s). See also Nicolas Terry, Prime Health: Should Amazon Purchase a Hospital Chain? MEDIUM (July 30, 2017), https://medium.com/@nicolasterry/prime-health-should-amazon-purchase-ahospital-chain-7a322d1ad0ec (contemplating the possibility of Amazon using its interest in wellness to experiment with providing health care). 
out facilities and personnel, win out, we may seem some serious bending of the cost curve albeit at the cost of professional influence over healthcare policy.

Finally, some other properties of Value will be implicated not just in the near term but far longer. The most obvious concern data protection. AI diagnosis will be performed almost invisibly, with our devices and wearables streaming data to cloud-based algorithms. Most of the ethical and legal issues have already been discussed. One issue, however, deserves re-emphasis. AI diagnosis arguably involves or triggers the type of data determinism that most disturbs us. There, value and empathy will not be in tension but hand-in-hand will view automation with concern and skepticism.

Far more obvious tensions exist between Automation represented by AI diagnostics and Empathy. By way of background the concept of diagnosis and its replacement by $\mathrm{AI}$ is open to criticisms based on oversimplification. First, reliance on the impressive amounts of data streaming off apps, wearables, and IoT devices neglects some of the subtler diagnostic data. For example, Jerome Groopman observes, "Doctors typically begin to diagnose patients the moment they meet them." ${ }^{362}$ This is because "they are interpreting a patient's appearance: his complexion, the tilt of his head, the movements of his eyes and mouth, the way he sits or stands up, the sound of his breathing." ${ }^{363}$ Obviously, this raises the question whether sensor-collected data will be sufficient for diagnoses. To an extent these are heuristics, diagnostic short-cuts, and the literature is highly critical of the incorrect applications of heuristics. ${ }^{364}$ Yet, these subjective interpretations by the physician of the of the state of the patient also reflect (or should reflect) the empathy that underpins the physician-patient relationship. It may be that the implicit and explicit cues that lead to these interpretations not only aide in the diagnosis but also reassure the patient and improve communication and disclosure of valuable information.

A second objection to the automation described here is that it supposes a somewhat simplified distinction between diagnosis and treatment. A physician might well argue that she starts treating a patient as soon as they meet. In passing, it is interesting that the legal system appears to accept a sharper divide as there is authority that the "schools of thought" defensive instruction in medical malpractice cases applies only to treatment cases, not those alleging negligent diagnosis. ${ }^{365}$ More importantly, the empathy value may in some part be based on

362. Jerome Groopman, What's the Trouble?, NEW YORKER (Jan. 29, 2007), http://www.newyorker.com/magazine/2007/01/29/whats-the-trouble $I$.

363. Id.

364. See Diagnostic Errors, PATIENT SAFETY NETWORK, https://psnet.ahrq.gov/primers/primer/12/diagnostic-errors (last visited Junc 8, 2017) (explaining that while heuristics are extremely useful, researchers have raised awareness to several types of errors that "clinicians commonly make due to incorrect applications of heuristics").

365. See Morganstein v. House, 547 A.2d 1180, 1183 (Pa. 1988) (finding the jury instruction on different schools of medical thought to be erroneous in regards to whether appellee should have diagnosed 
an indivisible continuum of care that begins with the construction of a physicianpatient relationship and continues thereafter through both diagnosis and treatment.

\section{CONCLUSION}

Will the "New" Triangle of Automation, Value, and Empathy be immutable like Kissick's? The simple answer is "of course not." This new triangle is more" of a conceit, designed to focus discussion on some very difficult and interlinked policy questions that will be raised by healthcare data technologies coming on stream over the next couple of decades. The special nature of the original iron triangle and the key to its longevity in health policy discussion is its immutability. The original triangle is perfect because it has proven unbeatable. Whether working with a taxpayer-based, single-payer, universal coveragehealthcare system such as in the United Kingdom or a hybrid public-private, market-driven system such as in the U.S., policymakers are faced with the unassailable nature of Kissick's construct.

Mine is not the first "new" triangle. Just as automation drove my model so the shift from individuated clinical care to public health and population health led to the Triple Aim, that focused the goals of health policy on "improving the individual experience of care; improving the health of populations; and reducing the per capita costs of care for populations." 366 Although the points of that triangle initially seem framed as compatible goals they also involve trade-offs. "For example, improving care for individuals can raise costs if the improvements are associated with new, effective, but costly technologies or drugs. Conversely, eliminating overuse or misuse of therapies or diagnostic tests can lead to both reduced costs and improved outcomes." The situation is made more complex by time delays among the effects of changes. Good preventive care may take years to yield returns in cost or population health. ${ }^{367}$ Notwithstanding, and in the words of Bill Sage, "Unlike Kissick's iron triangle of cost, access, and quality goals in eternal competition with one another, Berwick's triad is synergistic and capable

his patient's condition as unstable angina); $c f$. Wasfi v. Chaddha, 588 A.2d 204, 210 (Conn. 1991) (concluding that despite the unfortunate use of "schools of thought," the trial court adequately conveyed the settled principle that a treatment need only be one of a choice among competent physicians); Kowalski v. Marymount Hosp., Inc., 2007-Ohio-828U, No. CV-513-687, 2007 WL 613865 (Ohio Ct. App. 2007) (holding that the "different methods" jury instruction is misleading to the jury when assessing whether the physician failed to diagnose a particular disease from the observed symptoms); D'Angelis v. Zakuto, 556 A.2d 431,434(Pa. 1989) (concluding the schools of medical thought jury instruction, without elaboration, was erroneous in assessing a proper diagnosis);

366. Berwick et al., supra note 131 , at $760-62$.

367. Id. at 760 . 
of simultaneous realization. ${ }^{368}$ Hopefully, the same will be said about Automation, Value, and Empathy.

The new triangle makes two modest contributions. First, it updates our conception of "Quality" to "Value" by not only embracing the IOM's quality properties but also by including contemporary quality concerns such as data protection and evolving approaches to judging quality such as comparative effectiveness research and cost effectiveness analysis. Second, and arguably of greater value it introduces Empathy into the discussion of healthcare trade-offs. Long-term, Empathy's counterpoint to Automation will help us determine whether what will turn out to be massive investments in healthcare data technologies have been worthwhile.

The rise of Automation is beyond rapid and the second machine age will impact every industry and profession even while simultaneously beguiling us with new consumer-facing platforms and apps. However, just as the Triple Aim is bedeviled by timing ("Good preventive care may take years to yield returns in cost or population health." ${ }^{369}$ ), so it may take a decade or two to see how Automation, Value, and Empathy relate to each other and gain an appreciation of which tensions can be massaged out of the way and which others (to finish on a dystopian note) will persist, creating fundamental disharmony between man and medical machine.

368. William Sage, Physicians and the New Health Care Industry-Benefits of Generational Change, HEALTH AFF. BLOG (Mar. 1, 2016), http:/healthaffairs.org/blog/2016/03/01/new-health-caresymposium-physicians-and-the-new-health-care-industry-benefits-of-generational-change/.

369. Berwick et al., supra note 131 , at 760 . 
